University of Louisville

ThinkIR: The University of Louisville's Institutional Repository

Electronic Theses and Dissertations

$12-2016$

\title{
The effects of direct peritoneal resuscitation on leukocyte infiltration after acute brain death.
}

Jessica Lee Weaver

University of Louisville

Follow this and additional works at: https://ir.library.louisville.edu/etd

Part of the Physiological Processes Commons

\section{Recommended Citation}

Weaver, Jessica Lee, "The effects of direct peritoneal resuscitation on leukocyte infiltration after acute brain death." (2016). Electronic Theses and Dissertations. Paper 2603.

https://doi.org/10.18297/etd/2603

This Doctoral Dissertation is brought to you for free and open access by ThinkIR: The University of Louisville's Institutional Repository. It has been accepted for inclusion in Electronic Theses and Dissertations by an authorized administrator of ThinkIR: The University of Louisville's Institutional Repository. This title appears here courtesy of the author, who has retained all other copyrights. For more information, please contact thinkir@louisville.edu. 
THE EFFECTS OF DIRECT PERITONEAL RESUSCITATION ON LEUKOCYTE INFILTRATION AFTER ACUTE BRAIN DEATH

\author{
By \\ Jessica Lee Weaver \\ B.S., University of Virginia, 2007 \\ M.D., Medical College of Wisconsin, 2011
}

\begin{abstract}
A Dissertation in Partial Fulfillment of the Requirements for the Degree of

Doctor of Philosophy

in Physiology and Biophysics

Department of Physiology and Biophysics

University of Louisville

Louisville, Kentucky
\end{abstract}

Submitted to the Faculty of the School of Medicine of the University of Louisville

December 2016 

THE EFFECTS OF DIRECT PERITONEAL RESUSCITATION ON LEUKOCYTE INFILTRATION AFTER ACUTE BRAIN DEATH

\author{
By \\ Jessica Lee Weaver \\ B.S., University of Virginia, 2007 \\ M.D., Medical College of Wisconsin, 2011
}

A Dissertation Approved on:

October 10. 2016

By the following Dissertation Committee:

Jason Smith

Claudio Maldonado

Dale Schuschke

Stanley D'Souza

Brian Harbrecht 


\title{
DEDICATION
}

This dissertation is dedicated to my parents

\author{
Alfred C. Weaver \\ and \\ Debra L. Weaver
}

for their unfailing support

and in loving memory of

Jack B. Hilliard 


\section{ACKNOWLEDGEMENTS}

I would like to thank my mentor Dr. Jason W. Smith for his support and guidance. I would also like to thank the other committee members, Drs. Claudio Maldonado, Dale Schuschke, Stanley D’Souza, and Brian Harbrecht for their assistance over the past two years. I would like to express my gratitude to Dr. Kelly McMasters and the Department of Surgery for allowing me the time and giving me the financial support necessary to pursue this opportunity. Many thanks to my fellow lab members, including Dr. R. Neal Garrison, Dr. Cynthia Downard, Dr. Paul Matheson, Amy Matheson, Dr. Victoria Graham, Jessica Shepherd, and Dr. Jessica Raque. 


\title{
ABSTRACT \\ THE EFFECTS OF DIRECT PERITONEAL RESUSCITATION ON LEUKOCYTE INFILTRATION AFTER ACUTE BRAIN DEATH
}

\author{
Jessica L. Weaver
}

October 10, 2016

\section{$\underline{\text { Background }}$}

Brain death triggers an inflammatory response that is associated with worse outcomes for recipients of transplanted organs procured from brain dead donors. The intestine has been shown to release inflammatory mediators in other types of shock, but its role is brain death is not well described. Direct peritoneal resuscitation (DPR) improves visceral organ blood flow and has been shown to reduce inflammation after hemorrhagic shock. Better understanding of this inflammatory process within the peripheral organs, the effects of DPR, and the role of the intestine could aid in better resuscitation of potential organ donors.

\section{$\underline{\text { Methods }}$}

Male Sprague-Dawley rats were made brain dead by inserting a 4F Fogarty catheter into the skull and slowly inflating the balloon. Rats were resuscitated with normal saline to maintain a mean arterial pressure of $80 \mathrm{mmHg}$ and DPR animals also received an intraperitoneal injection of commercial peritoneal dialysis solution. Rats were sacrificed at zero, two, four, and six hours after brain death. Protein levels were assessed using quantitative ELISA. Flow cytometry and immunohistochemistry were used to quantify 
neutrophil and macrophage infiltration.

\section{$\underline{\text { Results }}$}

Flow cytometry demonstrates that macrophages and neutrophils infiltrate the liver, lungs, kidneys, and heart between four and six hours after brain death. Brain death causes increases in serum levels of inflammatory mediators, as well as markers of intestinal injury, which are reduced by DPR. A detailed examination of the lungs and kidneys showing that inflammatory cytokines, leukocyte adhesion molecules, and macrophages and neutrophils increase sequentially within the tissue after brain death, and that DPR reduces every one of these inflammatory stages.

\section{$\underline{\text { Conclusions }}$}

These results suggest that multiple peripheral organs in rats experience an influx of leukocytes between four and six hours after brain death. It appears that increased intestinal permeability contributes to this. Using DPR affects every step of this process by maintaining intestinal blood flow, reducing the release of these pro-inflammatory signals, reducing adhesion molecule expression, and ultimately decreasing the macrophages and neutrophils that move into the tissues. Use of DPR during resuscitation of brain dead patients has the potential to reduce systemic inflammation and provide better organs for transplant. 
TABLE OF CONTENTS

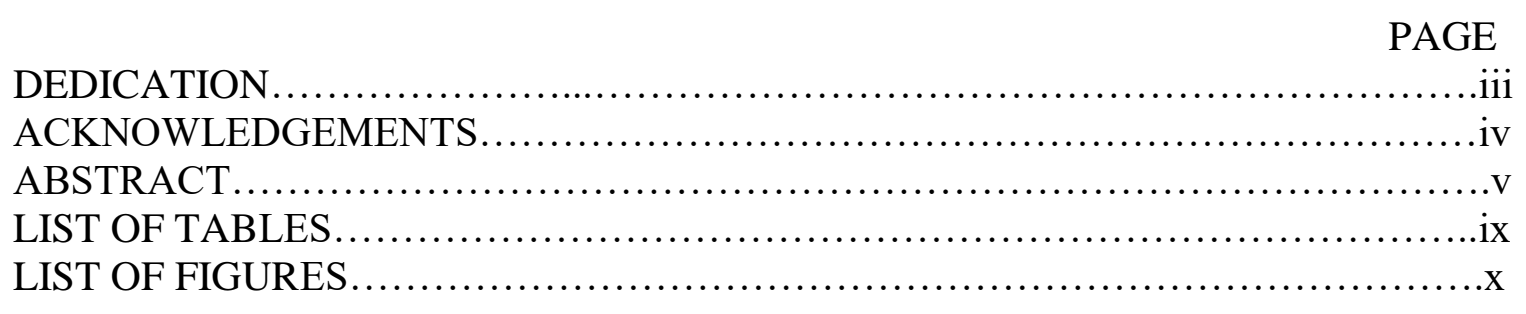

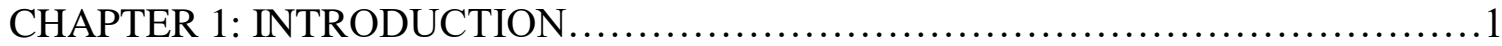

Traumatic Brain Injury...............................................

Physiology of Brain Death and Resuscitation.............................

Brain Death and Organ Donation....................................... 3

Inflammation in Brain Death........................................... 3

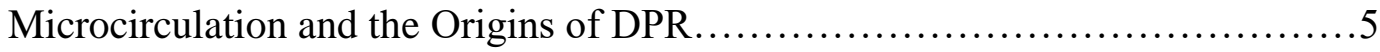

Cellular Mechanism of DPR...........................................6

Direct Peritoneal Resuscitation in Shock................................. 7

Direct Peritoneal Resuscitation in Acute Brain Death..........................8

Cytokines and Leukocytes............................................

Leukocytes and Organ Transplantation..................................9

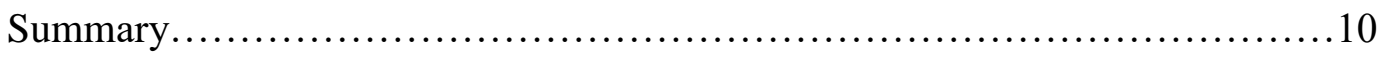

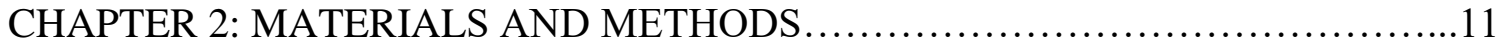

CHAPTER 3: HYPOTHESES AND SPECIFIC AIMS............................ 16

CHAPTER 4: TIMELINE OF NEUTROPHIL AND MACROPHAGE INFILTRATION

INTO ORGANS AFTER ACUTE BRAIN DEATH............................ 18

Overview....................................................... 18 


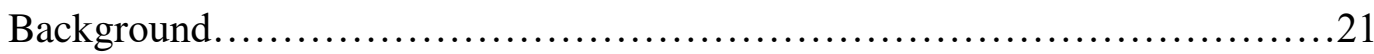

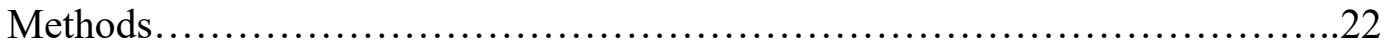

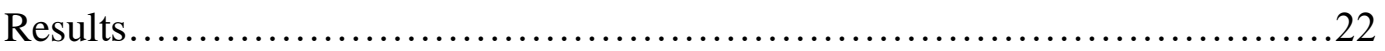

Discussion...................................................................

Conclusions .................................................................. 45

CHAPTER 5: THE EFFECT OF DIRECT PERITONEAL RESUSCITATION ON

LEUKOCYTE ACTIVITY IN THE LUNG .......................................47

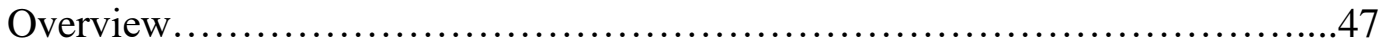

Background ............................................................. 49

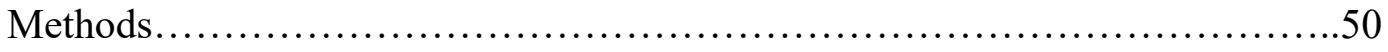

Results................................................................ 50

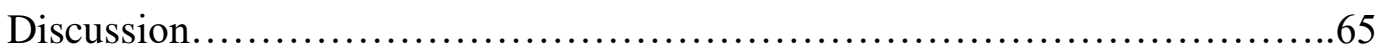

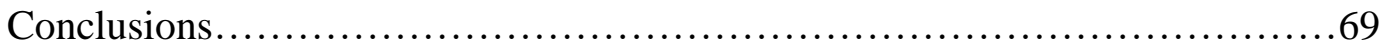

CHAPTER 6: THE EFFECT OF DIRECT PERITONEAL RESUSCITATION ON

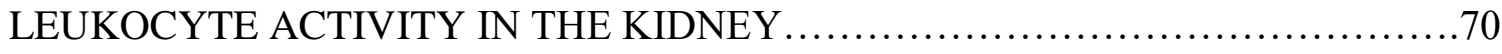

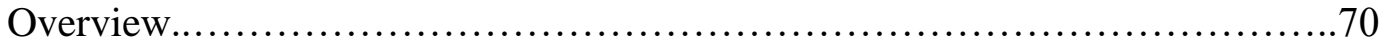

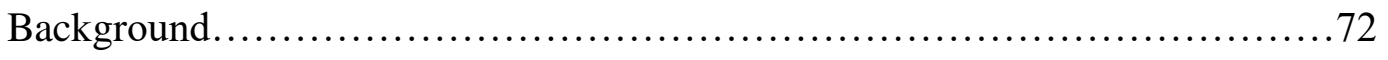

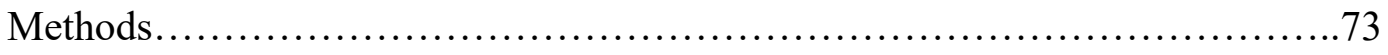

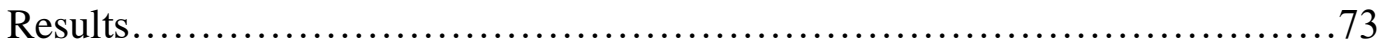

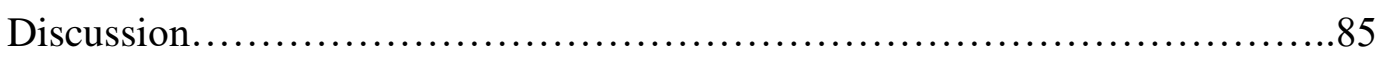

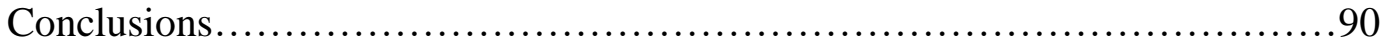

CHAPTER 7: CONCLUSIONS ............................................. 91

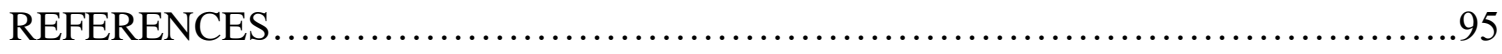

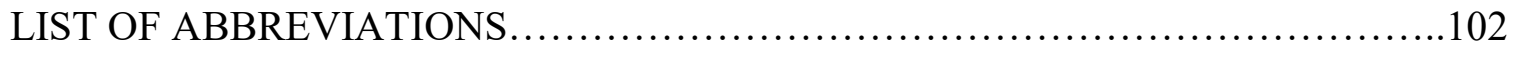

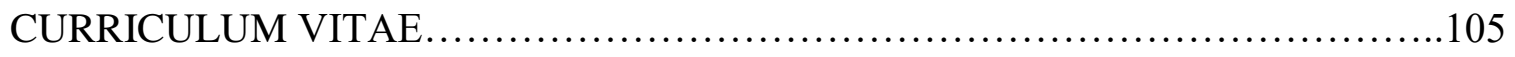




\section{LIST OF TABLES}

TABLE

PAGE

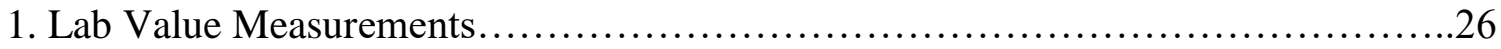

2. Serum Cytokine Measurements.......................................... 36

3. Lung Cytokine Measurements............................................... 51

4. Lung Pathology Descriptions...........................................64

5. Kidney Cytokine Measurements.......................................... 84 


\section{LIST OF FIGURES}

$\begin{array}{lll}\text { FIGURE PAGE } & \text { PAR }\end{array}$

1. Heart Rate over Time...................................................23

2. Mean Arterial Pressure over Time..........................................24

3. Blood Granulocytes over Time.........................................27

4. Blood Monocytes over Time..........................................28

5. Blood Monocyte Activation over Time...................................29

6. Tissue Neutrophils over Time.......................................... 31

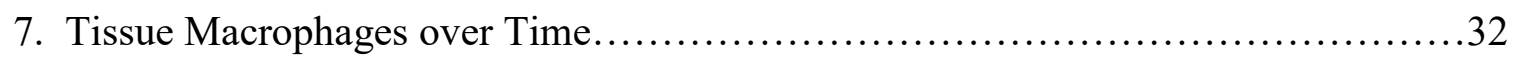

8. Tissue Macrophage M1 Activation....................................... 33

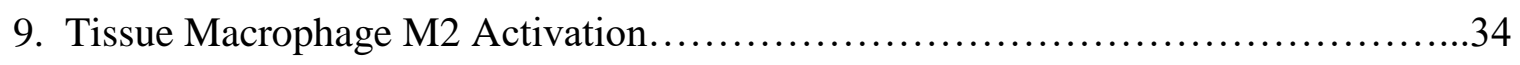

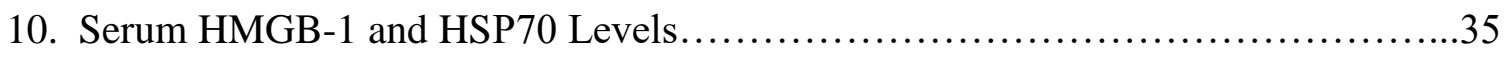

11. Serum FABP and LPS Levels......................................... 38

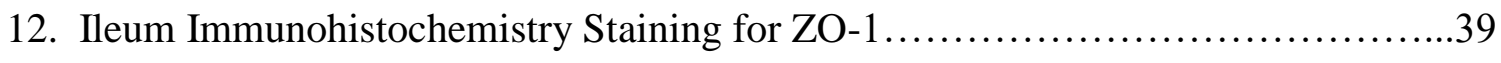

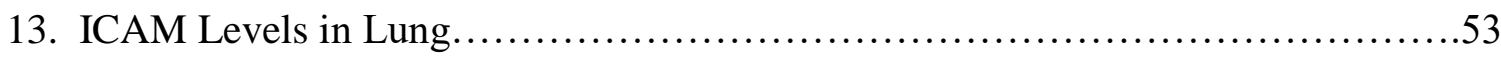

14. VCAM Levels in Lung................................................ 54

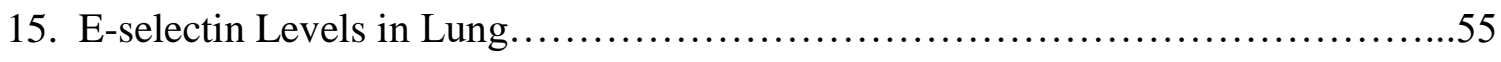

16. P-selectin Levels in Lung............................................. 56

17. Neutrophil Count in Lung.............................................57

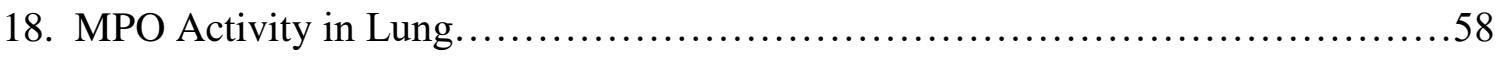

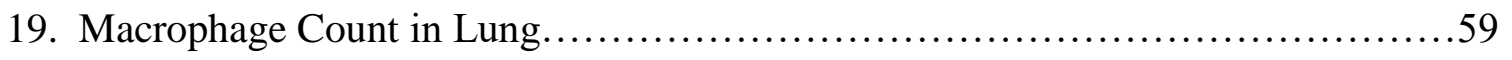


20. Macrophage Activation in Lung............................................60

21. Lung Immunohistochemistry Staining for Macrophages.........................62

22. Lung Immunohistochemistry Staining for Neutrophils........................63

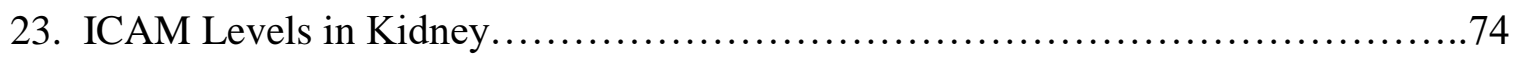

24. VCAM Levels in Kidney.................................................. 75

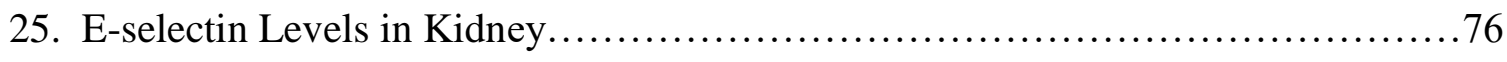

26. P-selectin Levels in Kidney..................................................77

27. Neutrophil Count in Kidney.................................................... 79

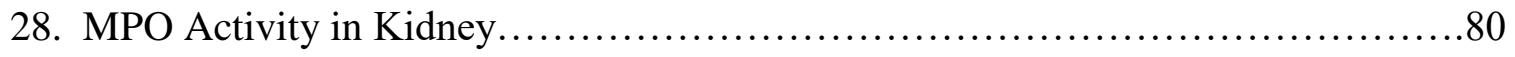

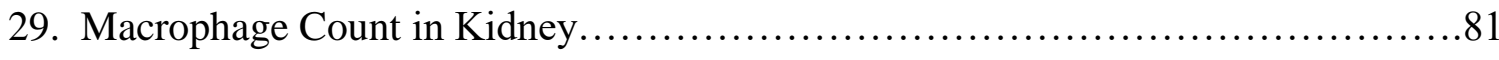

30. Macrophage Activation in Kidney ........................................... 82

31. Timeline of Leukocyte Infiltration and Effect of DPR..........................92 


\section{CHAPTER 1}

\section{BACKGROUND AND REVIEW OF THE LITERATURE}

\section{Traumatic Brain Injury}

An average of 1.7 million people sustain a traumatic brain injury (TBI) in the United States every year. Of these, 275,000 are hospitalized and 52,000 die [1]. It has been estimated that a moderate TBI can incur over $\$ 2.5$ million, and treatment of a severe injury can cost over $\$ 5$ million [2]. Thus, TBIs remain a significant cause of morbidity, mortality, and economic burden in the United States.

The severity of TBI extends across a spectrum. A concussion is a mild form of TBI which is defined as a rapid, short-lived impairment of normal neurological function which normally resolves in 7-10 days [3]. A persistent vegetative state, commonly referred to as a coma, is a severe injury causing a lack of higher brain function. These patients are unaware of their surroundings and have no motor or cognitive function, but respiration and circulation remain intact. In contrast, brain death is equivalent to death, and includes a loss of spontaneous respiration and all other brain stem functions, in addition to the complete lack of cortical brain function [4].

\section{Physiology of Brain Death and Resuscitation}

Brain death occurs when a person suffers a TBI or stroke that leads to bleeding around or inside the brain which causes an increase in intracranial pressure (ICP). In addition, the hypoxia and hypotension that frequently accompany traumatic injury lead to reduced oxygen delivery to brain cells. This leads to a reduction in adenosine 
triphosphate (ATP) levels and subsequent dysfunction of ATP-dependent ion pumps. This causes electrolyte imbalances that lead to cellular edema, apoptosis, and release of inflammatory mediators, worsening this cellular swelling [5]. As the brain tissue expands in the enclosed space of the skull this increases ICP, pushing the brain downward through the foramen magnum, compressing the brain stem and causing ischemia and infarction. As the brain stem dies, the patient undergoes significant physiological changes. First there is an intense increase in sympathetic outflow. This leads to a rise in catecholamine levels, causing peripheral vasoconstriction and increased heart rate and blood pressure, leading to increased myocardial oxygen demand and pulmonary hypertension. This increased oxygen demand combined with coronary vasoconstriction can cause cardiac ischemia, arrhythmias, and cardiac dysfunction. Following this "sympathetic surge" there is a complete loss of sympathetic outflow, leading to profound peripheral vasodilation and hypotension. In addition there are significant endocrine changes leading to low levels of thyroid hormone, antidiuretic hormone, and insulin, leading to significant electrolyte abnormalities, worsening hypotension, and hyperglycemia [6]. Therefore, prompt and aggressive support of these patients is necessary to prevent cessation of cardiac function.

The focus of resuscitation in brain death is maintaining blood pressure in order to maintain organ perfusion. This frequently involves the infusion of large volumes of intravenous (IV) fluids. This helps replace the water loss after the development of diabetes insipidus and also helps maintain blood pressure and organ perfusion. Ionotropes such as epinephrine or norepinephrine may also be used to support blood pressure, however these can worsen cardiac function. Endocrine supplementation with 
insulin, thyroid hormone, steroids, and vasopressin further support blood pressure and prevent electrolyte irregularities. Mechanical ventilation is also necessary as respiratory function ceases after brain death [6-8]. Supporting blood pressure and ventilation in these ways helps maintain organ perfusion and tissue oxygenation, preserving organ function.

\section{Brain Death and Organ Donation}

Resuscitation of brain dead patients is important because brain dead subjects have the potential to become organ donors. Organ transplantation is frequently the best or only option for end-stage organ failure. As the world's population has increased and aged, the number of patient's awaiting organ transplanted has rapidly outgrown the number of available organs. Between 2009 and 2013 there were an average of 28,533 solid organ transplants in the United States [9], and worldwide in 2011 there were over 112,000 . However, this represents only about $10 \%$ of the current demand [10], and as many as 17 people per day die while on the waiting list [8]. Thus, different strategies are being developed to encourage organ donation, increase the number of organs transplanted from an individual donor, and also to improve the longevity of organs after transplantation in order to prevent the need for a single patient to be placed back on the list for repeat transplantation.

\section{Inflammation in Brain Death}

The purpose of resuscitation is also to prevent inflammation and multiple organ system failure, even in patients with isolated brain injuries. A mild or moderate response is normal and likely beneficial as part of the body's stress response to trauma, but an exaggerated response can lead to early organ failure $[11,12]$. When multiple organ 
failure develops, mortality can be anywhere between 30 and 100\%, depending on the number of organs involved [13]. Research has demonstrated that any type of trauma can trigger a profound inflammatory response, which is largely is mediated by the intestine. This became evident after increasing numbers of patients underwent surgical exploration (or autopsy) for presumed intra-abdominal sepsis, only to find no identifiable source of infection. This led to the hypothesis that bacteria from the intestine were moving into the blood stream and exacerbating organ dysfunction, a process known as "bacterial translocation" [14]. This can occur because hypotension and shock lead to increased vascular permeability and edema in the visceral organs, causing sloughing of the intestinal mucosa and diminishing both the absorptive and barrier functions of the gut [13]. Not only does ischemia lead to dysfunction of endothelial cell tight junctions, which normally prevent the movement of bacteria and large molecules across the endothelial barrier, but also promotes the activation of gut-associated lymphoid tissue and augments the body's inflammatory response [14]. The movement of bacteria, bacterial byproducts, or its associated endotoxin into the blood, as well as the exaggerated inflammatory response, can cause worsening hypotension and shock, resulting in a downward spiral that results in organ failure and death. Improving intestinal blood flow in shock may reduce inflammation and improve outcomes.

Brain death is associated with significant inflammation, though the role of the intestine as a source of this inflammation has not been well-studied. In an animal model of isolated brain death without other traumatic injury, there is a significant upregulation of systemic inflammatory mediators, as well as increased inflammatory cytokines within the peripheral organs $[15,16]$. Most often this inflammation is hypothesized to come 
from the brain itself $[16,17]$ or from endothelial activation [18]. If the intestine were to

play a role, targeting intestinal perfusion could reduce the inflammation associated with brain death.

\section{Microcirculation and the Origins of DPR}

Peritoneal dialysis fluid is vasodilatory [19]. Intravital microscopy studies have demonstrated that application of commercial peritoneal dialysis fluid to the terminal ileum causes rapid and sustained vasodilation of five different levels of arterioles. Further study revealed that this dilation was due to the presence of hypertonic solution and did not occur with isotonic solution. In addition, arteriolar vasodilation was independent of the $\mathrm{pH}$ of the solution [20].

This observation led to the hypothesis that use of hyperosmotic solution in the abdomen during shock would help improve blood flow to the visceral organs, help maintain intestinal barrier function, and reduce the body's inflammatory response. This procedure is known as direct peritoneal resuscitation (DPR). Use of colorimetric microspheres showed that use of DPR improved blood flow not only to the ileum, but also to the jejunum, pancreas, spleen, psoas muscle, diaphragm, and lung [21]. Subsequent experimentation using in vivo microscopy in rats demonstrated that use of DPR improves blood flow to the terminal ileum and reduces organ edema compared to conventional IV resuscitation, even though normal hemodynamic measurements were achieved in both groups [22-24].

More recent human studies have demonstrated that use of adjunctive DPR after hemorrhagic shock improves the time to abdominal closure, rate of primary fascial closure, and reduces abdominal complications [25]. Use of DPR in patients suffering 
from septic shock reduced time on the ventilator and in the intensive care unit (ICU), reduced abdominal complications, and improved mortality [26]. Use of DPR in brain dead organ donors improved organ edema and hepatic blood flow, leading to improved organ procurement rate [27].

\section{Cellular Mechanism of DPR}

The observed changes in blood flow from using DPR are associated with alterations on the cellular level. Normally hemorrhage leads to prolonged low blood flow leads to reduced oxygen delivery to the tissues. This leads to lower production of ATP, which in turn causes dysfunction of the ion exchange pumps that require ATP to function. This then leads to disruption of the electrochemical gradient across the cell membrane, causing water to enter the cell, leading to cellular swelling and cell damage [23]. The vascular endothelium is particularly vulnerable to an ischemia/reperfusion injury, especially the intestinal vasculature [28]. This dysfunction not only affects the permeability of this barrier, but also leads to a release of inflammatory mediators, increased expression of adhesion molecules, changes in vascular tone [29]. This loss of barrier function results in increased movement of fluid and proteins out of the intravascular space into the interstitial space, and increased expression of adhesion molecules leads to increased movement of leukocytes into the tissues [30]. This causes organ edema and dysfunction, and can result in multi-system organ failure and death.

The function of DPR is to prevent this process. By dilating the vasculature in shock, blood flow is maintained, particularly to the visceral organs. Thus, oxygen delivery and ATP production are maintained. This maintains the cellular electrochemical gradient and avoids endothelial cell damage, reducing the release of inflammatory 
mediators and expression of adhesion molecules. Thus peritoneal resuscitation not only works macroscopically by maintaining visceral organ blood flow, but also works on the cellular level to prevent the release of mediators which would otherwise cause organ dysfunction.

\section{Direct Peritoneal Resuscitation in Shock}

Extensive study using a rat model revealed numerous benefits of the use of DPR during resuscitation after hemorrhagic shock. Both animals that received conventional IV fluid resuscitation (CR) alone and those that received DPR showed the expected drop in blood pressure during hemorrhage, and return to baseline with resuscitation $[21,22$, 24]. However, CR animals experienced progressive intestinal vasoconstriction, whereas DPR was associated with dilation of the visceral vasculature and increased splanchnic blood flow [24]. Use of DPR also resulted in less edema formation in the liver, spleen, and lung [22].

The fact that an application of DPR to the abdominal cavity caused changes in lung edema suggests that DPR does not work merely through direct contact, but is having truly systemic effects. This improvement in visceral blood flow leads to a reduction in a number of inflammatory mediators released in response to shock. In an experiment comparing animals that received a sham procedure (no hemorrhage) to those which received CR or DPR, serum levels of inflammatory cytokines IL-1 $\alpha$, IL-1 $\beta$, IL-6, IL-17, and IL-18 were equivalent to sham levels in the DPR animals and both were significantly lower than those in the CR group [31, 32].

Peritoneal resuscitation also exerted effects on damage-associated molecular patterns (DAMPs). These represent a diverse group of molecules which act much like 
external pathogens, activating toll-like receptors that initiate the body's inflammatory response [33, 34]. For example, High-Mobility Group Box 1 protein (HMGB-1) is a DAMP which helps facilitate transcription and is normally sequestered in the nucleus due to its powerful inflammatory properties [35-38]. Serum levels of HMGB-1 were increased in the CR group and equivalent to sham levels in the DPR group. Immunohistochemistry (IHC) staining further demonstrated that HMGB-1 remained in the nucleus in the DPR animals while migrating to the cytosol in the CR animals [smith thesis]. Both of these findings indicate that DPR helps reduce inflammation and preserve cell structure. Hyaluronic acid (HA) is another DAMP which is found throughout the body, particularly in connective tissue [39]. Hyaluronan also forms a part of the connections between cells, and elevated levels in blood have been linked to sepsis, cirrhosis, and other serious conditions [39, 40]. Serum levels of HA were also increased in the CR group and reduced in the DPR group [smith thesis]. This implies that use of DPR not only decreases the production of these inflammatory mediators, but also helps maintain tight junction integrity, reducing the permeability of the endothelial cells and preventing leakage of DAMPs into the blood.

\section{Direct Peritoneal Resuscitation in Acute Brain Death}

Direct peritoneal resuscitation has also been applied to a rat model of acute brain death. In these experiments, rats that received targeted IV fluid (TIVF) resuscitation to maintain a mean arterial pressure (MAP) of $80 \mathrm{mmHg}$ were compared to those that received TIVF and DPR. Liver blood flow in the DPR group was higher than that in the TIVF group. In addition, this result was achieved with significantly less IVF. This translated directly into a reduction in edema in the lung, liver, and ileum. Similar to what 
was observed in hemorrhagic shock, DPR after acute brain death was also associated with a reduction in inflammatory cytokines including IL-1 $\alpha$, IL-1 $\beta$, IL-4, IL-6, IL-13, IL-18, and IFN- $\gamma[41]$.

\section{Cytokines and Leukocytes}

These changes in cytokine levels may correlate with changes in leukocyte activity, as numerous cytokines act on leukocytes, specifically macrophages and neutrophils, to recruit and activate them. Neutrophils have been shown to secrete TNF- $\alpha$, IL-1 $\beta$, TGF- $\beta$, IFN- $\gamma$ as well as IL-6, IL-8, IL-10, IL-12 [42, 43]. Monocytes (immature macrophages) have also been shown to produce TNF- $\alpha$, TGF- $\beta$, and IL-8 [43]. Activated macrophages produce numerous cytokines including IL-1, IL-6, IL-12, and TNF- $\alpha$ [44]. The endothelium also produces a number of cytokines which are used to attract immune cells after injury, such as IL-1 $\alpha$, IL-6, IL-8, TNF- $\alpha$ [45]. Macrophages and neutrophils activate and modulate each other's activity, as well as that of other immune cells, such as T cells $[46,47]$. Therefore, the changes in cytokine levels seen with the addition of DPR suggests that there may be a modulation of the underlying immune response.

\section{Leukocytes and Transplantation}

The activity of leukocytes is extremely important in acute brain death because the success of organ transplantation is linked to leukocyte activity. When an organ is transplanted, resident macrophages from the donor are transplanted within the organ tissue. These remain in place and reproduce for approximately 4 weeks, after which they start to decline. The recipient's macrophages also move into the organ within 24 hours of transplantation and begin to proliferate [48]. However, while the presence of resident macrophages is normal, significant accumulation of macrophages within the transplanted 
organ is associated with organ acute and chronic organ rejection [48-50], which results in reduced graft survival.

Neutrophils also play an important role in the immune response after transplantation. Neutrophils accumulate in the lungs only a few hours after an ischemiareperfusion injury [50], and studies of cardiac allografts demonstrate prolonged survival in recipients lacking the neutrophil chemokine receptor CXCR2 [51]. Neutrophils also work in conjunction with macrophages, complementing and activating each other, to protect the body from pathogens $[46,47]$. Striking a balance between leukocyte activation and suppression after transplantation is difficult, delicate work as leukocytes are necessary for wound healing and prevention of infection, but over-activation of macrophages and neutrophils can lead to organ rejection.

\section{Summary}

Previous evidence links DPR to the actions of leukocytes in shock. Use of DPR causes vasodilation of the microvasculature in the visceral organs after shock. This improves blood flow, reducing the permeability of the intestine during shock and quells the release of inflammatory mediators. This may, in turn, cause reduced movement of leukocytes into organs as well as reduced activation of those cells. This reduction in leukocytes could ultimately result in organs which have lower rejection rates and better outcomes after transplantation. This goal of this project was the better delineate the course of inflammation and leukocyte infiltration into organs that occurs after acute brain death in the rat and determine the effects of DPR upon this process. 


\section{CHAPTER 2}

\section{MATERIALS AND METHODS}

Animals were maintained at the Veterans Affair Hospital Research Facility in Louisville, KY which is approved by the American Association for the Accreditation of Laboratory Animal Care. This protocol was approved by the Institutional Animal Care and Use Committee. All experiments were performed using male Sprague-Dawley rats weighing $250-300 \mathrm{~g}$. The individual rats used in each group were chosen using a random number generator iPhone application. They were given food and water $a d l i b$ and were acclimated using 12 hour light/dark cycles for at least 7 days prior to use.

Anesthesia was initiated with intraperitoneal (IP) pentobarbital $(50 \mathrm{mg} / \mathrm{kg})$ and maintained with subsequent IP injections of $10-25 \%$ of the original dose. Body temperature was maintained at $37.0 \pm 1.0^{\circ} \mathrm{C}$ with a rectal probe and a servo-controlled heating pad. Once loss of withdrawal reflexes was confirmed, an incision was made along the anterior surface of the neck. The trachea was isolated, a tracheotomy made and a tracheostomy inserted (Intramedic PE-240 polyethylene tubing, Clay Adams Division of Becton Dickinson \& Company, Parsipanny, NJ). Animals were allowed to spontaneously breathe room air. Then the right carotid artery was isolated and cannulated with PE-50 tubing. This was then connected to the monitor to record continuous heart rate (HR) and mean arterial pressure (MAP). The right internal jugular vein was cannulated and used to administer intravenous fluids. The rat was then placed prone and a vertical incision made in the skin between the eyes. The skull was cleared of 
connective tissue and cleaned with acetone. A drill was then used to create a small opening in the outer layer of the skull. Forceps were used to remove the inner layer of the skull and enter the epidural space. A 4F Fogerty catheter was inserted slowly into this space with the balloon completely deflated. The rat was then returned to the supine position and allowed to equilibrate for 20 minutes.

Baseline MAP and HR measurements were taken 10 minutes apart after the equilibration period was complete. After the second measurement was taken the epidural catheter was inflated at a rate of $1 \mathrm{cc} / \mathrm{hr}$. During the inflation period MAP and HR were recorded every 5 minutes. When the sympathetic surge was seen, the tracheostomy was connected to the ventilator and the rat was ventilated at a tidal volume of $1.5 \mathrm{cc}$ and a rate of 90 breaths/minute. If at any point during the balloon inflation the rat began displaying signs of respiratory distress, the ventilator was connected at that time. Once the MAP began to drop, IV normal saline (NS) was infused as necessary to maintain a MAP of 80 mmHg. Animals scheduled for DPR received a single IP injection of 30cc of $2.5 \%$ glucose-based clinical peritoneal dialysis solution (Delflex ${ }^{\circledR}$, Fresenius USA, Inc. Ogden, UT) that contains $5.67 \mathrm{~g} / \mathrm{L}$ sodium chloride, $3.92 \mathrm{~g} / \mathrm{L}$ sodium lactate, $0.257 \mathrm{~g} / \mathrm{L}$ calcium chloride, $0.152 \mathrm{~g} / \mathrm{L}$ magnesium chloride at a $\mathrm{pH}$ of 5.5 and osmolality of $398 \mathrm{mOsm} / \mathrm{L}$ ). Peritoneal resuscitation solutions were pre-warmed to $37^{\circ} \mathrm{C}$ prior to injection.

Animals were sacrificed at the time of resuscitation (time zero), two, four, or six hours post-resuscitation. Sham animals underwent cannula placement and the drilling of the hole in the skull but did not undergo the brain catheter placement or inflation. They were sacrificed at 2 hours. At the time of sacrifice the lung, liver, heart, spleen, kidney, and ileum were frozen in liquid nitrogen. Samples of lung and ileum were preserved in 
Normal Buffered Formalin (NBF) for histology. Samples of liver, kidney, heart, and lung were also preserved for flow cytometry. For this, $250 \mathrm{mg}$ of lung tissue and $500 \mathrm{mg}$ of kidney, heart, or liver tissue was cut into small pieces, then allowed to digest with a $0.1 \%$ collagenase solution for 30 minutes at $37^{\circ} \mathrm{C}$. The solution was then strained through a $70 \mu \mathrm{m}$ cell strainer to remove excess tissue. The solution was centrifuged and the cells resuspended in DPBS solution to remove excess enzyme solution. This was centrifuged again and resuspended in a 1\% paraformaldehyde (PFA) solution and allowed to fix for 20 minutes at room temperature. The cells were then washed with DPBS and resuspended and stored in $1 \mathrm{~mL}$ of a solution of $70 \%$ DPBS, $20 \%$ calf serum, and $10 \%$ glycerol and frozen at $-80^{\circ} \mathrm{C}$.

To prepare samples for flow cytometry, they were thawed and $500 \mu \mathrm{L}$ of the tissue digestion was stained using the Foxp3 Staining Buffer Set (eBioscience). Antibodies used included Anti-CD163:FITC (Bio-Rad); Anti-CD68:Alexa Fluor 700 (Bio-Rad); Anti-CD80:APC (Life Technologies); Anti-CD11b/c:PE-Cy 7 (BD Bioscience); and Anti-RP-1:PE (BD Bioscience). Neutrophils were identified by the presence of CD11b and RP-1 [52, 53] and macrophages by CD68 [54]. Macrophage activation was represented CD80 for M1 activation and CD163 for M2 activation [54, 55]. Flow cytometry was formed using a Becton Dickinson LSR II detector with lasers at $405 \mathrm{~nm}, 488 \mathrm{~nm}$, and $633 \mathrm{~nm}$.

Whole blood was drawn at the time of sacrifice and a complete metabolic panel (CMP) was measured using the Abaxis VetScan VS2. For store prior to flow cytometry, whole blood was centrifuged and the supernatant serum was separated and frozen. The red blood cells were suspended in 1\% PFA, allowed to fix for 20 minutes at room 
temperature, washed with DPBS, and stored in the DPBS/calf serum/glycerol solution. To prepare blood for flow cytometry $100 \mu \mathrm{L}$ of blood were prepared using the same FoxP3 Staining Buffer Set and same antibodies as used in the tissue samples. Flow cytometry was formed using the same detectors with lasers at $405 \mathrm{~nm}, 488 \mathrm{~nm}$, and 633 $\mathrm{nm}$.

Analysis of protein levels was measured using quantitative ELISAs from LS Bio. In brief, tissues samples were lysed in buffer in the Tissue Lyser II. A dilution series was created using the available standard and buffer. Samples were then mixed in a 96-well plate with the provided buffer followed by substrate, washes, and stop solution according to the manufacturer's instructions. After the advised incubation period, plates were read at the recommended wavelength with a spectrophotometer. The dilution series was used to create a standard curve and the results were analyzed using SigmaPlot (Systat Software Inc).

To perform immunohistochemistry staining, samples were imbedded in paraffin, sectioned, and placed on slides. These were then stained with primary antibodies antiMPO for neutrophils, anti-CD68 for macrophages, and anti-ZO-1 (Abcam) using the Biocare system (Biocare Medical, LLC). Light microscopy was then used to evaluate the position of macrophages and neutrophils within the tissue. Tissue edema and damage was graded as mild, moderate, or severe by a blinded pathologist. The pathologist graded tissue edema and hemorrhage on a scale of mild, moderate, or severe and graded the quantity of macrophages and neutrophils as few or numerous.

Statistical analysis was performed on the heart rate, blood pressure, and lab values, as well as quantitative values obtained from the MPO assay, quantitative ELISA, 
and flow cytometry. Comparisons of heart rate, blood pressure, and lab values among all groups at the same time point were made using SPSS software (IMB) and an analysis of variance. Comparisons between all groups in the MPO assay and quantitative ELISA carried out on SigmaPlot software (Systat Software, Inc) using an analysis of variance. Comparisons of quantitative values on flow cytometry over time were made using an analysis of variance using SPSS, while direct comparisons between a time point with and without DPR used a student's t-test. 


\section{CHAPTER 3}

\section{HYPOTHESES AND SPECIFIC AIMS}

\section{Overall Hypothesis}

The use of Direct Peritoneal Resuscitation reduces the early migration of macrophages and neutrophils into abdominal and thoracic organs when compared to conventional resuscitation alone in animals subjected to acute brain death.

\section{Specific Hypotheses and Specific Aims}

Hypothesis \#1: Inflammation occurs rapidly after brain death, that increased intestinal permeability contributes to this inflammation, and that DPR will reduce systemic inflammation after brain death.

Specific Aim \#1: To create a timeline for inflammation and neutrophil and macrophage infiltration into the lung, liver, kidney, and blood, to study the permeability of the intestine over time, and how DPR affects intestinal permeability and subsequent inflammation in brain death.

Hypothesis \#2: Use of Direct Peritoneal Resuscitation reduces the infiltration of macrophages and neutrophils into the lung after brain death.

Specific Aim \#2: To evaluate the effect of DPR on the infiltration and activation of macrophages and neutrophils into the lung following acute brain death. Animals will be divided into 3 groups: 1) Sham (no brain death); 2) targeted IV fluid resuscitation 
(TIVF); 3) DPR (TIVF and 30cc intraperitoneal Delflex solution).

Hypothesis \#3: Use of Direct Peritoneal Resuscitation reduces the infiltration of macrophages and neutrophils into the kidney following brain death.

Specific Aim \#3: To evaluate the effect of DPR on the infiltration and activation of macrophages and neutrophils into the kidney following acute brain death. Animals will be divided into 3 groups: 1) Sham (no brain death); 2) targeted IV fluid resuscitation (TIVF); 3) DPR (TIVF and 30cc intraperitoneal Delflex solution). 


\section{CHAPTER 4}

\section{TIMELINE OF NEUTROPHIL AND MACROPHAGE INFILTRATION INTO ORGANS AFTER ACUTE BRAIN DEATH}

\section{Overview}

\section{$\underline{\text { Introduction }}$}

Brain death triggers a significant inflammatory response that is associated with worse outcomes for recipients of transplanted organs procured from brain dead donors. This inflammation has been hypothesized to arise from multiple sources, including the brain itself, or labile blood pressure causing ischemia/reperfusion injury and endothelial cell activation. The intestine has been shown to release inflammatory mediators in other types of shock, but its role is brain death has not been well-studied. Direct peritoneal resuscitation (DPR) improves visceral organ blood flow and has been shown to reduce inflammation after hemorrhagic shock. Better understanding of this inflammatory process and the role of the intestine could aid in better resuscitation of potential organ donors. We hypothesized that use of DPR would maintain intestinal integrity and reduce circulating inflammatory mediators after brain death. $\underline{\text { Methods }}$

Male Sprague-Dawley rats were anesthetized and had cannulas placed in the trachea, carotid artery, and internal jugular vein. Brain death was induced by inserting a 4F Fogarty catheter into the epidural space and slowly inflating it. After brain death was 
achieved, rats were ventilated and resuscitated with normal saline to maintain a mean arterial pressure of $80 \mathrm{mmHg}$. The DPR animals received a single injection of $30 \mathrm{~mL}$ intraperitoneal Delflex solution. Animals were sacrificed immediately (time aero), or at two, four, or six hours after brain death. Tissues were collected and snap frozen in liquid nitrogen or digested in collagenase to create a single cell suspension. Lab values were measured from whole blood using the VetScan VS2. Flow cytometry was performed using antibodies to CD11b and RP-1 for neutrophils and CD68 for macrophages. Protein levels were measured using quantitative ELISA and cytokines were measured using the MagPix Luminex cytokine array.

$\underline{\text { Results }}$

There were no differences in vital signs between the different groups. Compared to the time zero rats, rats at two, four, and six hours had increased sodium and decreased glucose, albumin, total protein, alkaline phosphatase, and amylase. Granulocytes or neutrophils were increased in the blood and all tissues as 6 hours compared to time zero, though only the blood, kidney, and lung reached statistical significance. Monocytes or macrophages were unchanged in blood and increased in all tissues at six hours, though only the kidney and heart reached statistical significance. Inflammatory cytokines IL-1 $\beta$ and IL-6 were increased at four hours, while damage associated molecular patterns HMGB-1 and HSP70 increased at two and six hours, respectively. Levels of multiple inflammatory cytokines including IL-1 $\beta$ and IL-6 as well as HMGB-1 and HSP70 were decreased with DPR. Fatty acid binding protein (FABP) and lipopolysaccharide (LPS), both markers of intestinal injury, were increased in the serum after brain death and decreased with DPR. Immunohistochemistry staining for ZO-1, a protein that forms a 
part of tight junctions, showed decreased tight junction integrity after brain death, as was improved with DPR.

\section{$\underline{\text { Conclusions }}$}

Our results demonstrate that brain death induces a rapid increase in a number of inflammatory mediators. This is likely in part due to an increase in intestinal permeability. These increased inflammatory mediators ultimately stimulate macrophages and neutrophils infiltrating the tissues at six hours. Using DPR prevent intestinal ischemia and downregulates the systemic inflammatory response. Procuring organs prior to six hours in rats, as well as therapies targeting at maintaining intestinal barrier function such as DPR, has the potential to create better organs for transplantation. 


\section{Background}

Brain death is associated with substantial physiologic changes, including myocardial and pulmonary dysfunction, as well as significant endocrine changes [6]. Brain death also leads to an increase in systemic inflammation, as well as elevated inflammatory mediators within the end organs [56-58]. This inflammation has been hypothesized to lead to worse outcomes when patients receive transplanted organs procured from brain dead donors compared to living donors or those deceased after cardiac death $[59,60]$. The cause of this inflammation is thought to arise from different sources. Some studies suggest that inflammation may arise from the brain tissue itself and leak into circulation due to damage to the blood-brain barrier $[16,17]$. The process of brain death is also associated with significant hemodynamic instability, including a rapid increase in circulating catecholamines and hypertension followed by profound peripheral vasodilation and hypotension [6]. This can lead to endothelial cell damage and upregulate inflammatory mediators [18]. However, while intestinal ischemia has been shown to be a significant source of inflammatory mediators in septic and hemorrhagic shock $[14,61,62]$, its role as a source in brain death has not been well described.

Numerous authors have examined levels of specific cytokines or leukocyte activity after brain death. However, these studies are generally limited to a specific organ, and to a single or only a few time points after brain death. They do not provide a true overall picture of how the process affects the body. A better understanding of the process of brain death could allow for improved resuscitation strategies to target inflammation. The purpose of this study was to create a more complete description of inflammatory activity after brain death in rats, including levels of cytokines, damage 
associated molecular patterns (DAMPs), and leukocyte infiltration into the liver, lung, kidney, and heart over time. Furthermore, this study is intended to determine the effects of brain death on intestinal permeability, and whether use of DPR would cause decreased changes in intestinal permeability, leading to reduced systemic inflammation.

\section{Materials and Methods}

The methods were performed as explained the Chapter 2: Materials and Methods. In brief, our murine model of brain death was used and rats were sacrificed immediately after brain death (time zero) as well as two, four, and six hours after brain death. Those scheduled for DPR received a single intraperitoneal injection of $30 \mathrm{~mL}$ of Delflex solution. After brain death rats were resuscitated with enough normal saline to maintain a MAP of $80 \mathrm{mmHg}$ and vitals were measured continuously throughout the experiment. After sacrifice, tissues were flash frozen in liquid nitrogen and ultimately used for protein analysis with ELISA, or digested in collagenase for creation of a single cell suspension for flow cytometry.

\section{Results}

The heart rate and mean arterial pressure of rats in each groups are displayed in Figures 1 and 2. All rats displayed the characteristic sympathetic surge in heart rate and blood pressure, and subsequent decrease associated with brain death. There were no significant differences among the groups at any time point during the resuscitation period. 


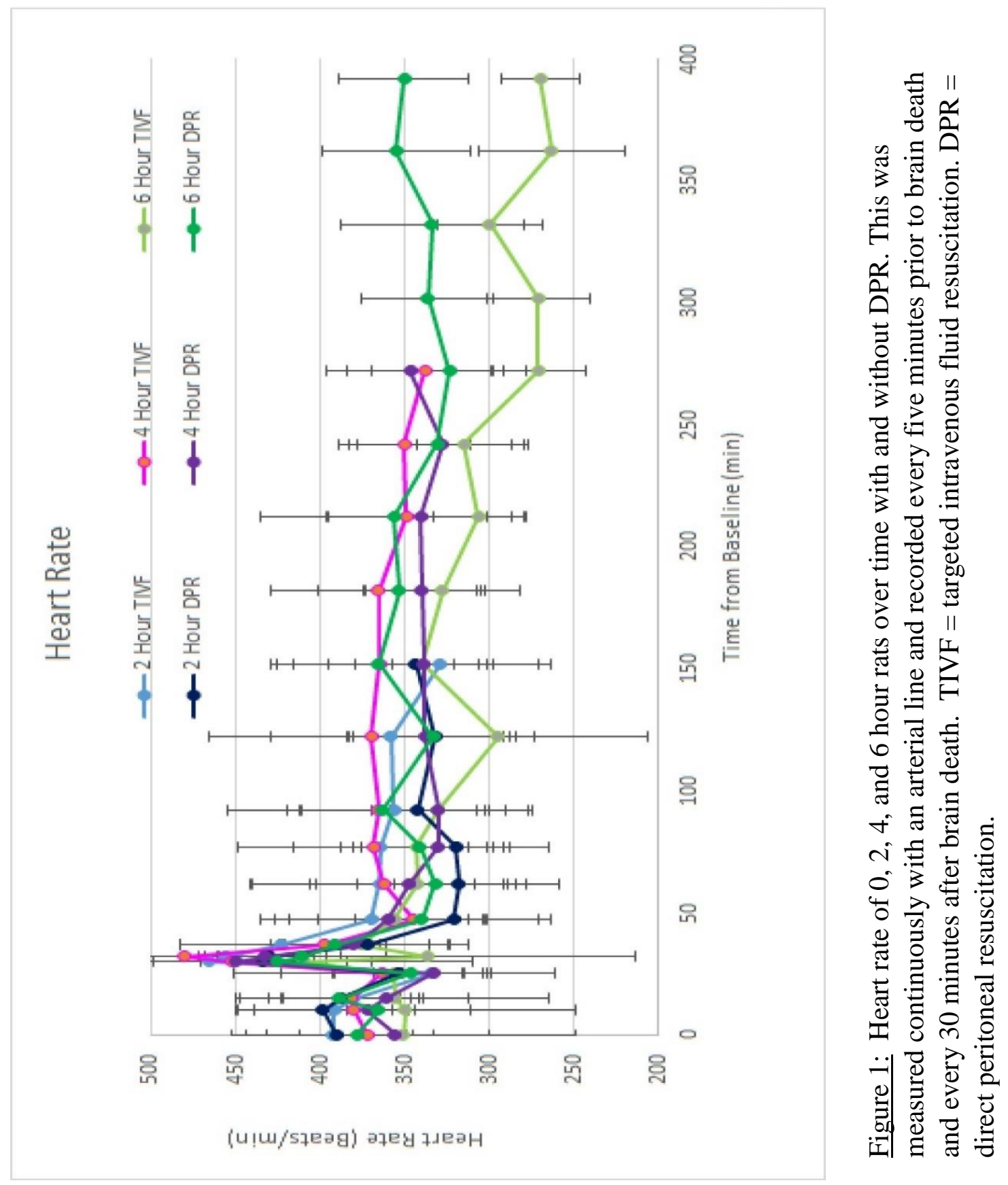




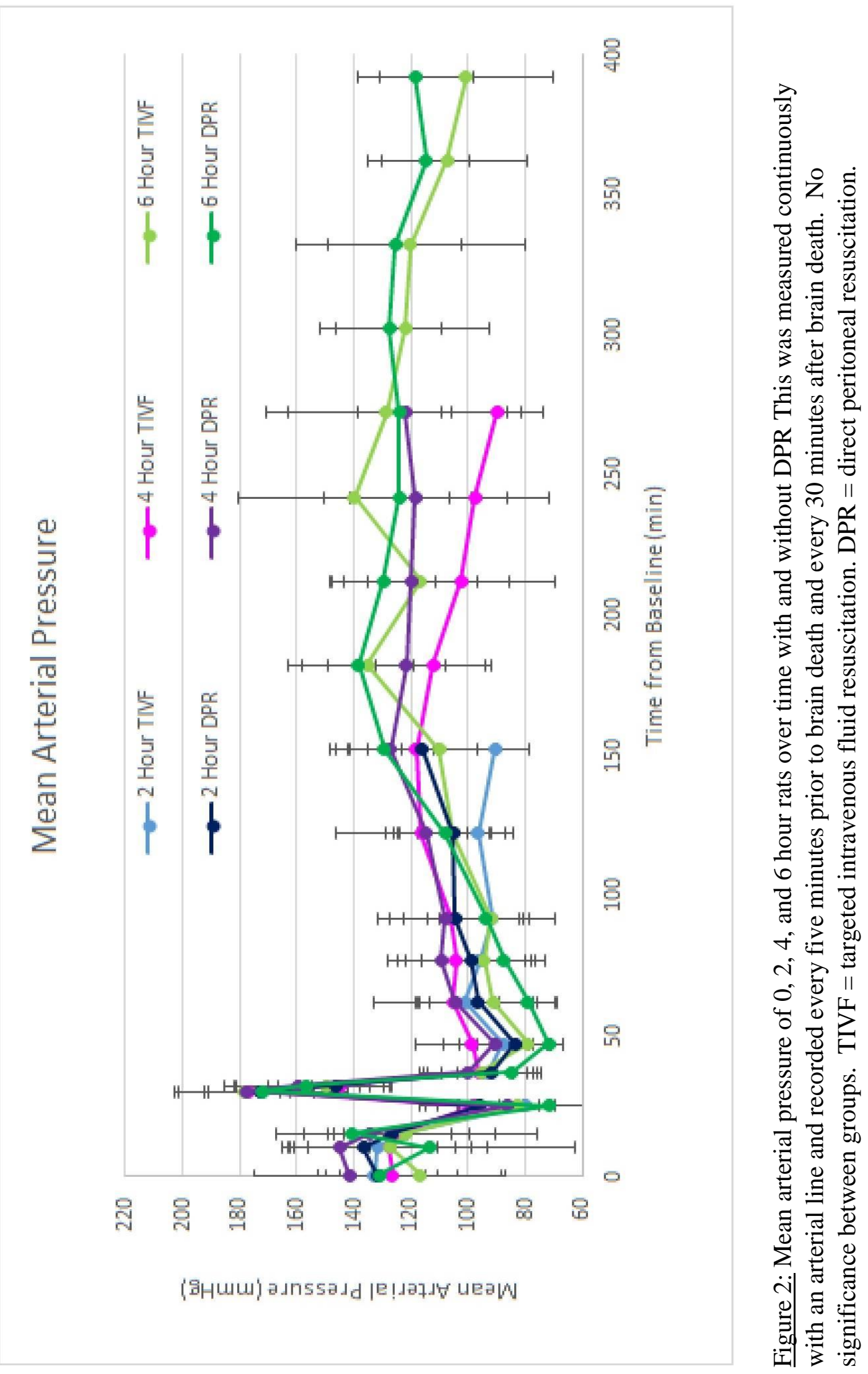


The results of a metabolic panel performed immediately after sacrifice is shown in Table 1. Serum levels of sodium were increased at two, four, and six hours compared to time zero. Sodium levels were lower in the two and six hour DPR groups compared to the two and six hour targeted IV fluid (TIVF) groups, respectively. Blood glucose was high immediately after brain death and returned to normal by two hours, while glucose was increased in the four hour DPR group compared to four hour TIVF. Alkaline phosphatase and total protein were decreased at two, four, and six hours compared to time zero. Amylase was decreased at four and six hours compared to time zero, and decreased at six hours compared to two hours. Albumin was decreased at two, four, and six hours compared to time zero and was decreased at six hours compared to all time points. Amylase and total protein were increased in the four hour DPR group over four hour TIVF, while albumin was only significantly increased in two hour DPR compared to two hour TIVF. Total IVF was decreased in all DPR groups compared to the TIVF groups, but this only reached statistical significance at 2 and 4 hours.

Flow cytometry results in the blood are shown in figures 3-5. Granulocytes were increased at six hours compared to zero and four hours. Monocytes trended down but no changes reached statistical significance. Both M1 and M2 activation both trended upward over time but only the M2 activation increase reached statistical significance. 


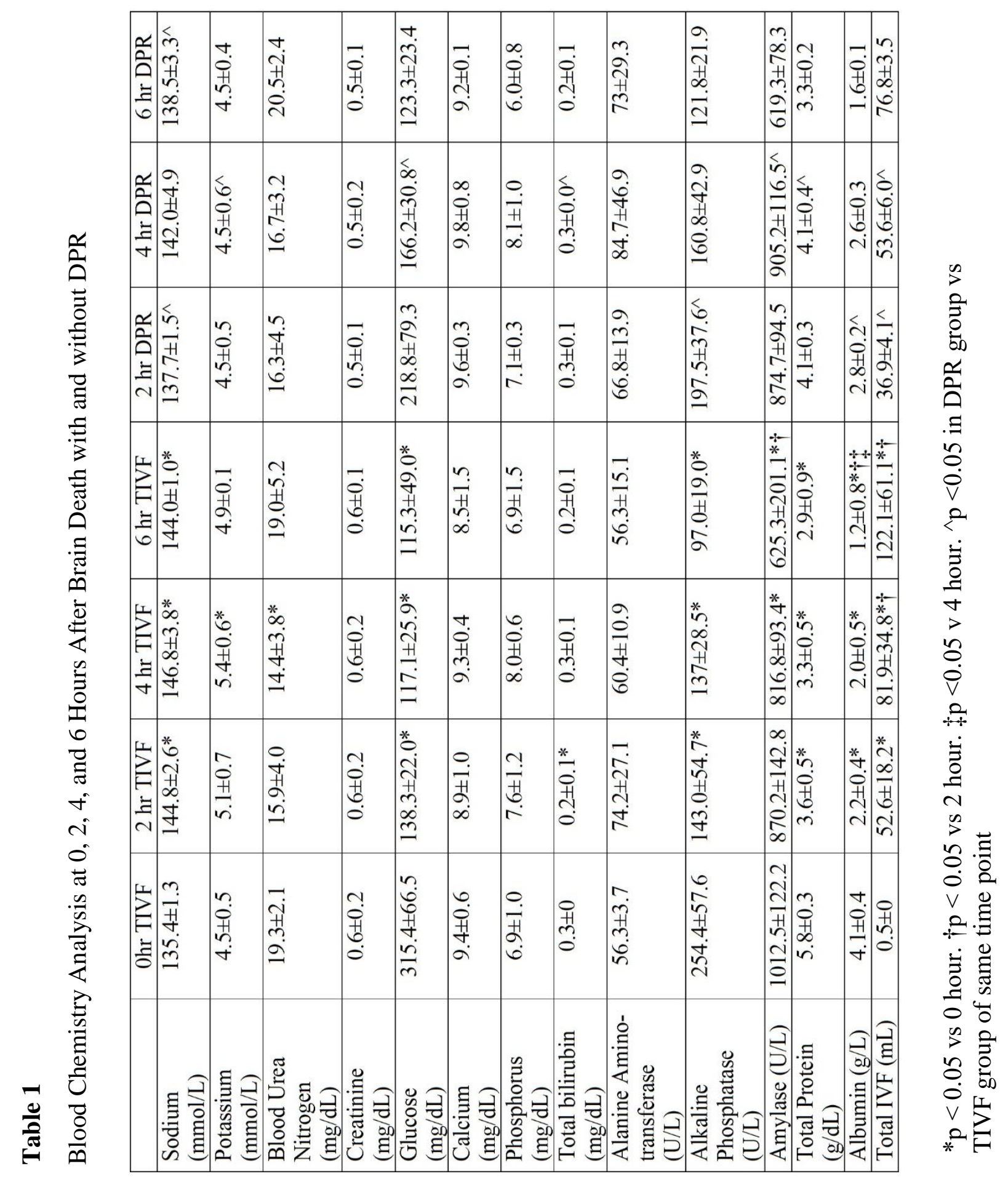




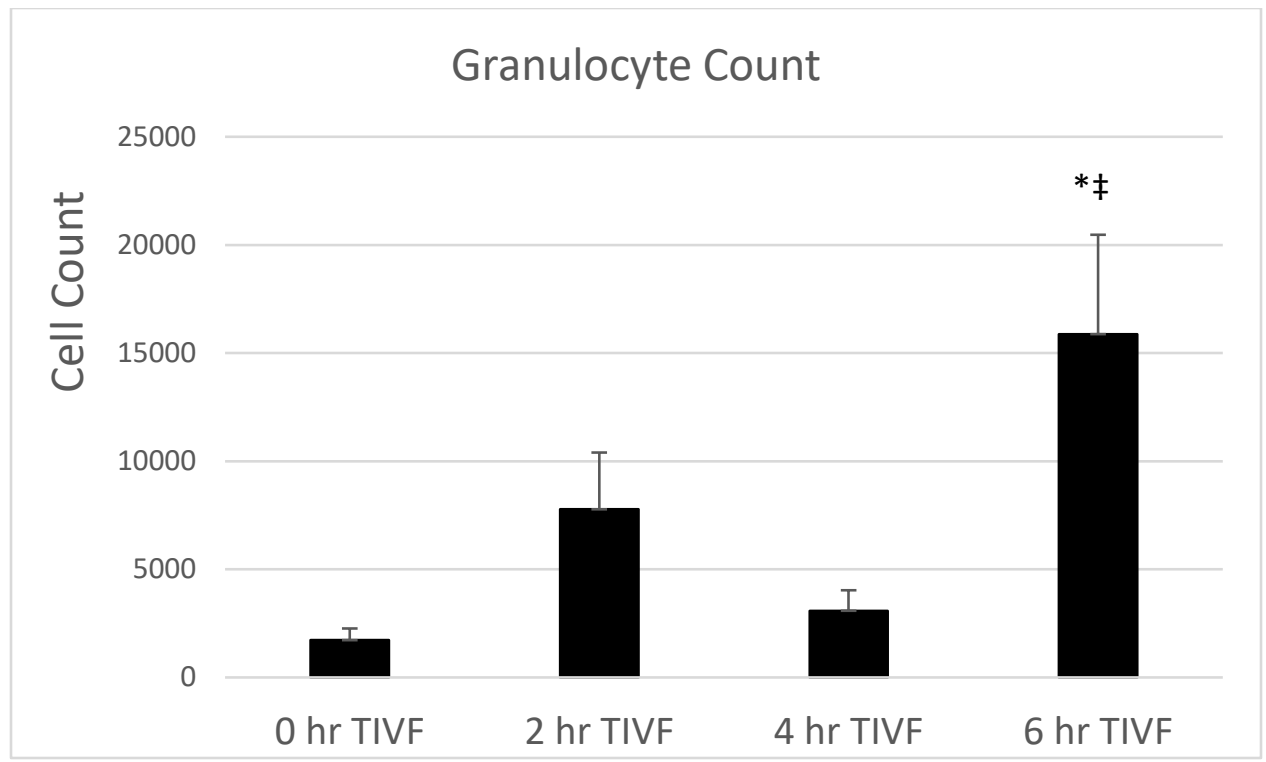

Figure 3: Granulocyte count in blood at 0, 2, 4 and 6 hours after brain death as measured by flow cytometry. $* \mathrm{p}<0.05$ vs 0 hour. $\uparrow \mathrm{p}<0.05$ vs 2 hour. $\$ \mathrm{p}<0.05$ v 4 hour. 


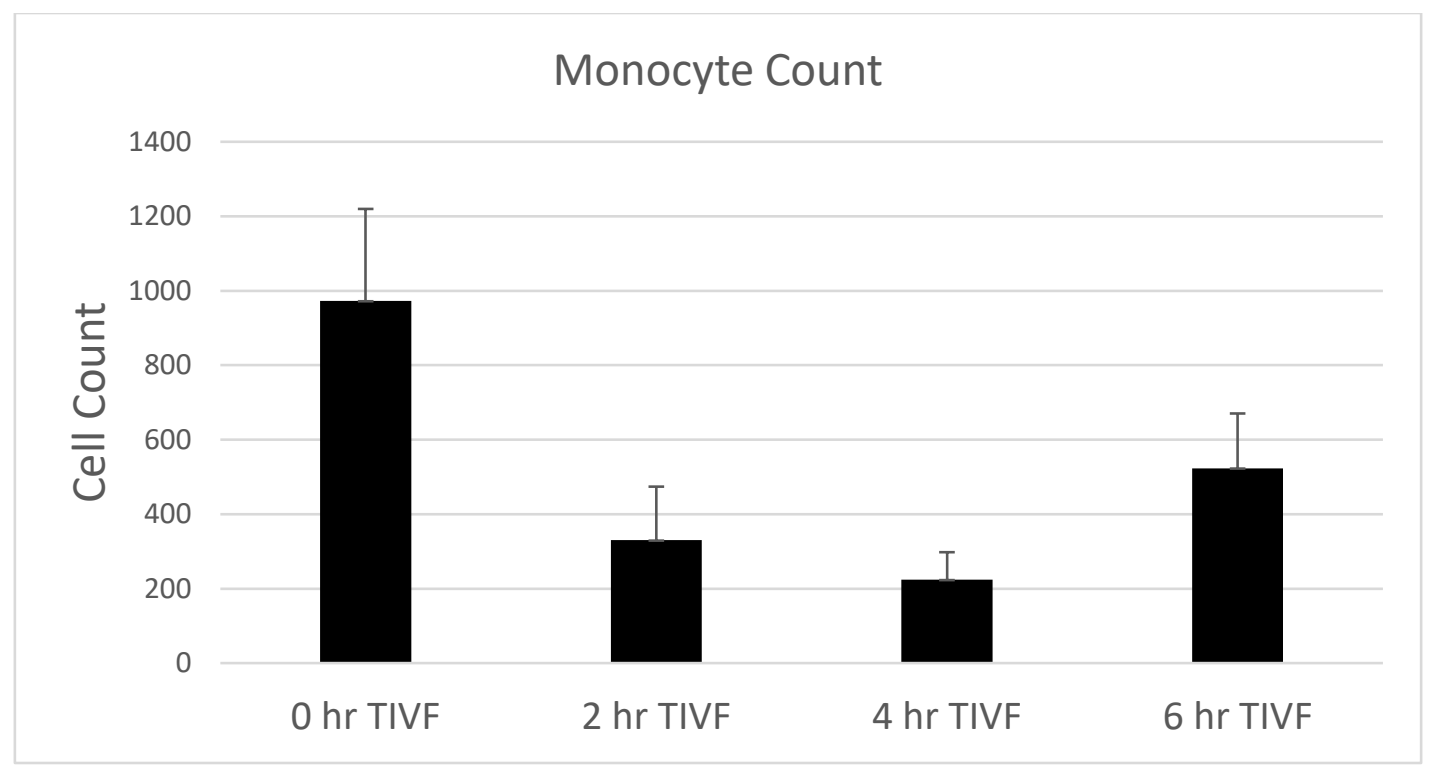

Figure 4: Monocyte count in blood at 0,2, 4 and 6 hours after brain death as measured by flow cytometry. No significance found between groups. 

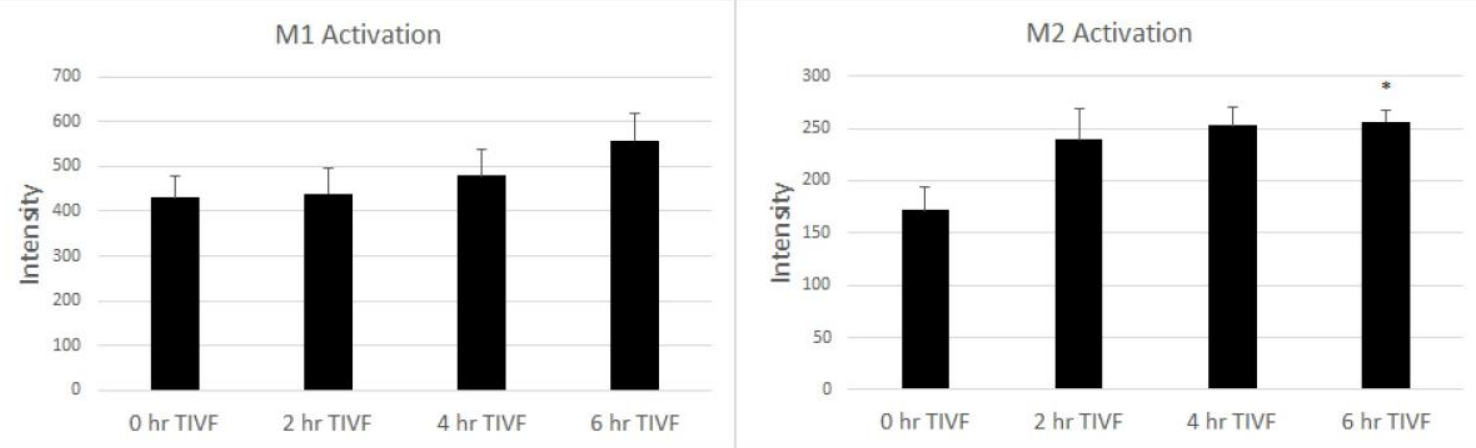

Figure 5: M1 and M2 activation of monocytes in blood at 0, 2, 4 and 6 hours after brain death. *p $<0.05$ vs 0 hour 
Flow cytometry results in the liver, lung, kidney, and heart tissue are shown in figures 6-9. Tissue neutrophils increased significantly at six hours in the kidney and lung. Tissue macrophages increased at six hours in the kidney and heart. Classical M1 activation was increased in all tissues by six hours, while M2 activation only increased significantly in the lung at four hours and decreased again at six hours.

Figure 10 displays results of ELISAs for serum levels of DAMPs high mobility group box 1 protein (HMGB-1) and heat shock protein 70 (HSP70). Levels of HMGB-1 peaked at two hours but were elevated at two, four, and six hours compared to sham and zero hours. Use of DPR significantly decreased HMGB-1 levels at all time points. Levels of HSP70 were increased at six hours compared to all other time points and all three DPR groups had significantly reduced HSP70 levels compared to TIVF alone. Serum levels of select inflammatory cytokines are shown in Table 2. Levels of TNF- $\alpha$, IL-4, IL-12, and IL-13 were unchanged over time. Protein levels of IL-1 $\alpha$ was increased by DPR at four and six hours, while IL- $1 \beta$ and IL-6 were increased at two, four, and six hours compared to sham or time zero and decreased with DPR. Anti-inflammatory IL-10 was increased at four and six hours and decreased with DPR, and IFN- $\gamma$ decreased over time. 


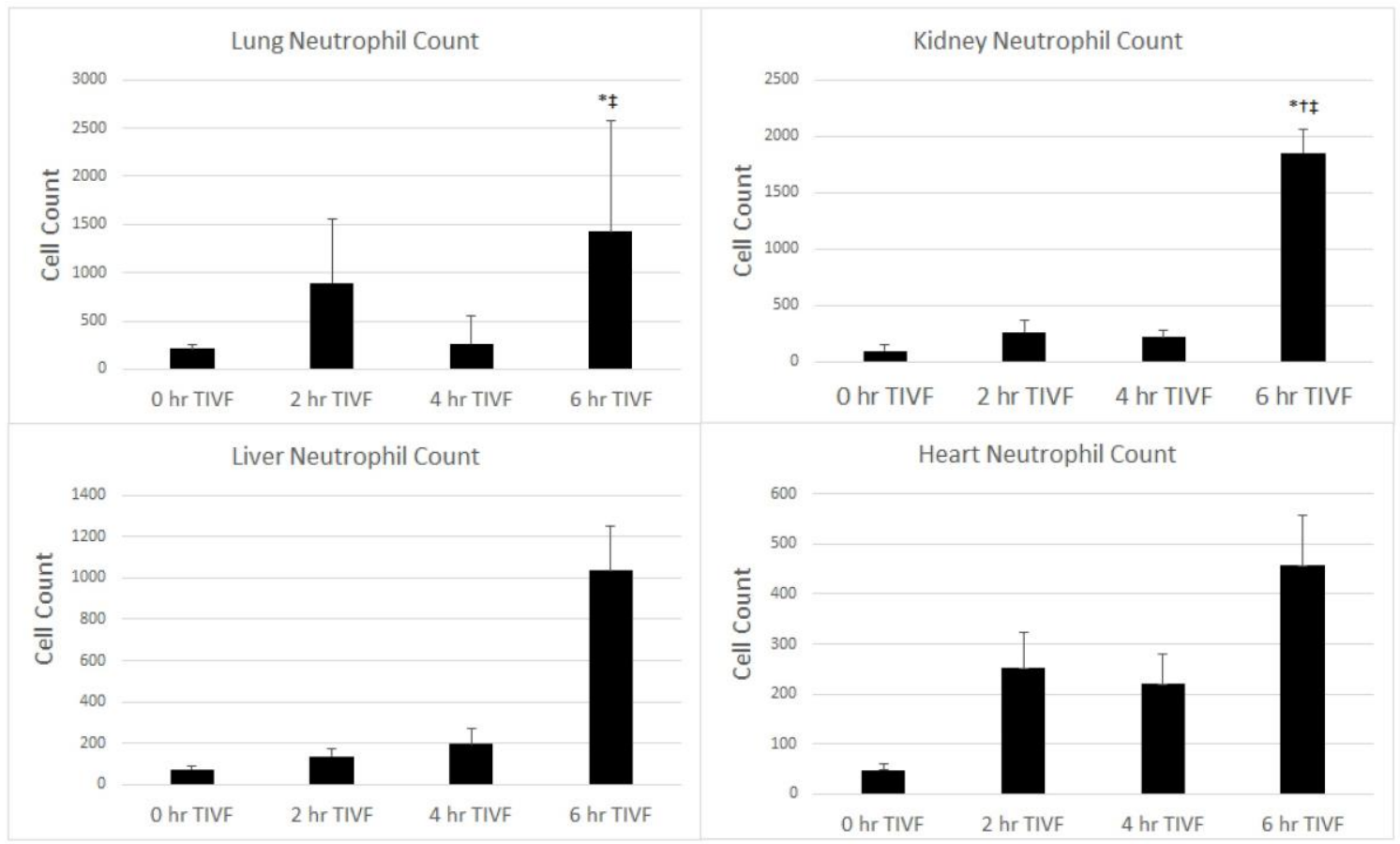

Figure 6: Neutrophil count in liver, lung, kidney, and heart at 0,2, 4 and 6 hours after brain death as measured by flow cytometry. ${ }^{*} \mathrm{p}<0.05$ vs 0 hour. $\dagger \mathrm{p}<0.05$ vs 2 hour. $\ddagger \mathrm{p}$ $<0.05 \mathrm{v} 4$ hour. 


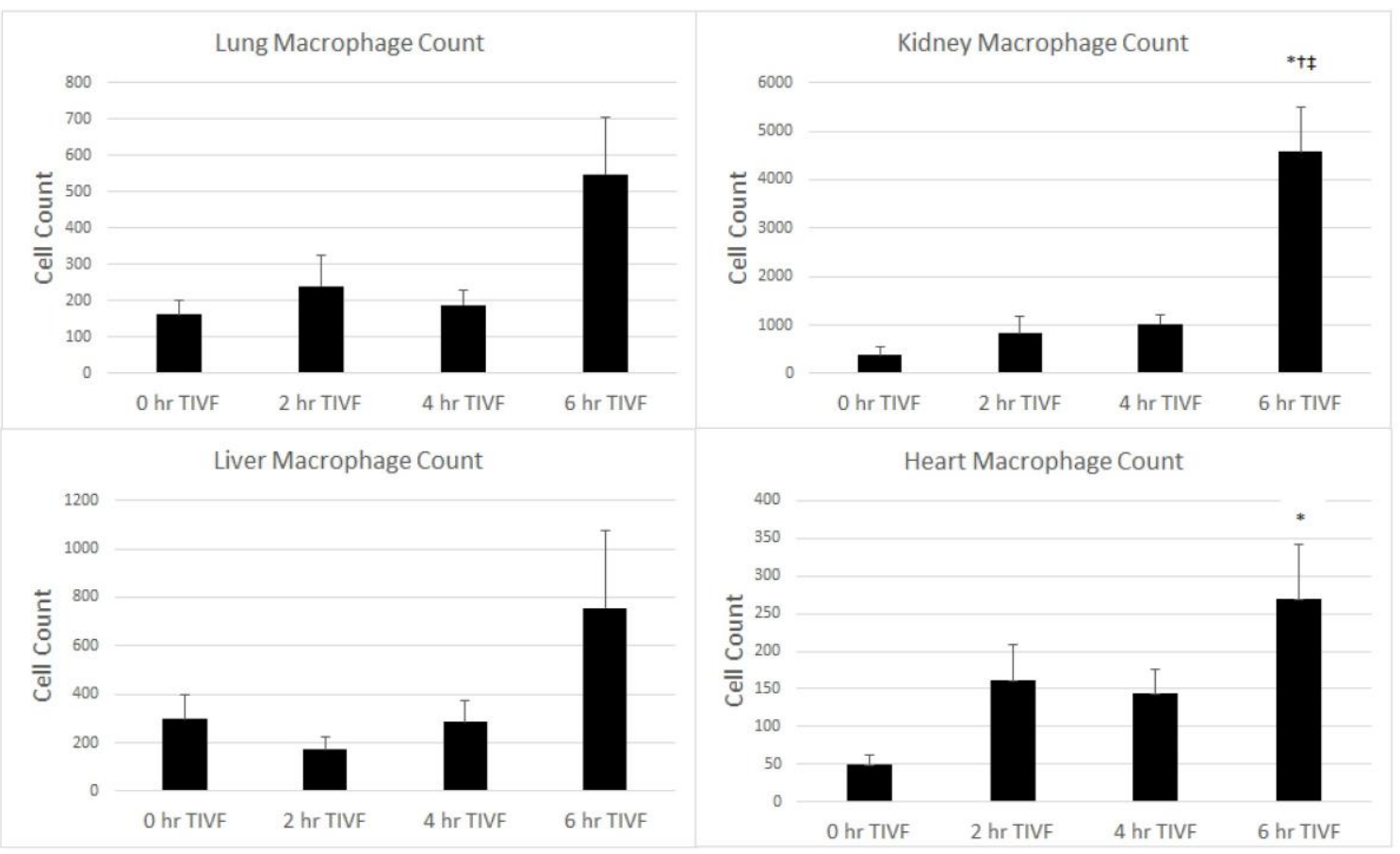

Figure 7: Macrophage count in liver, lung, kidney, and heart at 0, 2, 4 and 6 hours deviation as measured by flow cytometry. $* \mathrm{p}<0.05$ vs 0 hour. $\uparrow \mathrm{p}<0.05$ vs 2 hour. $\$ \mathrm{p}$ $<0.05$ v 4 hour. 


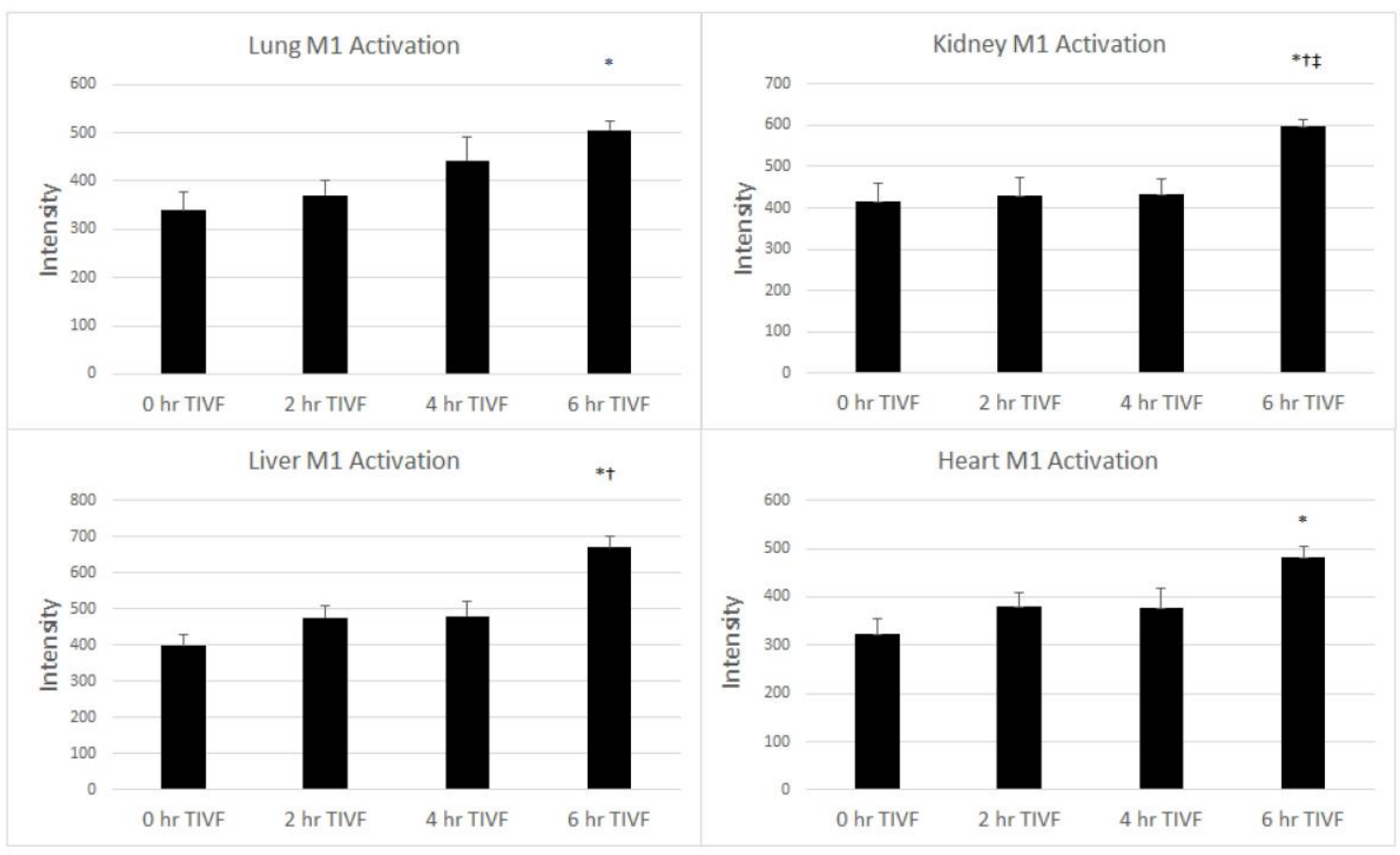

Figure 8: Intensity of M1 macrophage activation in liver, lung, kidney, and heart at 0, 2, 4 and 6 hours as measured by flow cytometry. ${ }^{*} p<0.05$ vs 0 hour. $\uparrow p<0.05$ vs 2 hour. $\$ p$ $<0.05$ v 4 hour. 


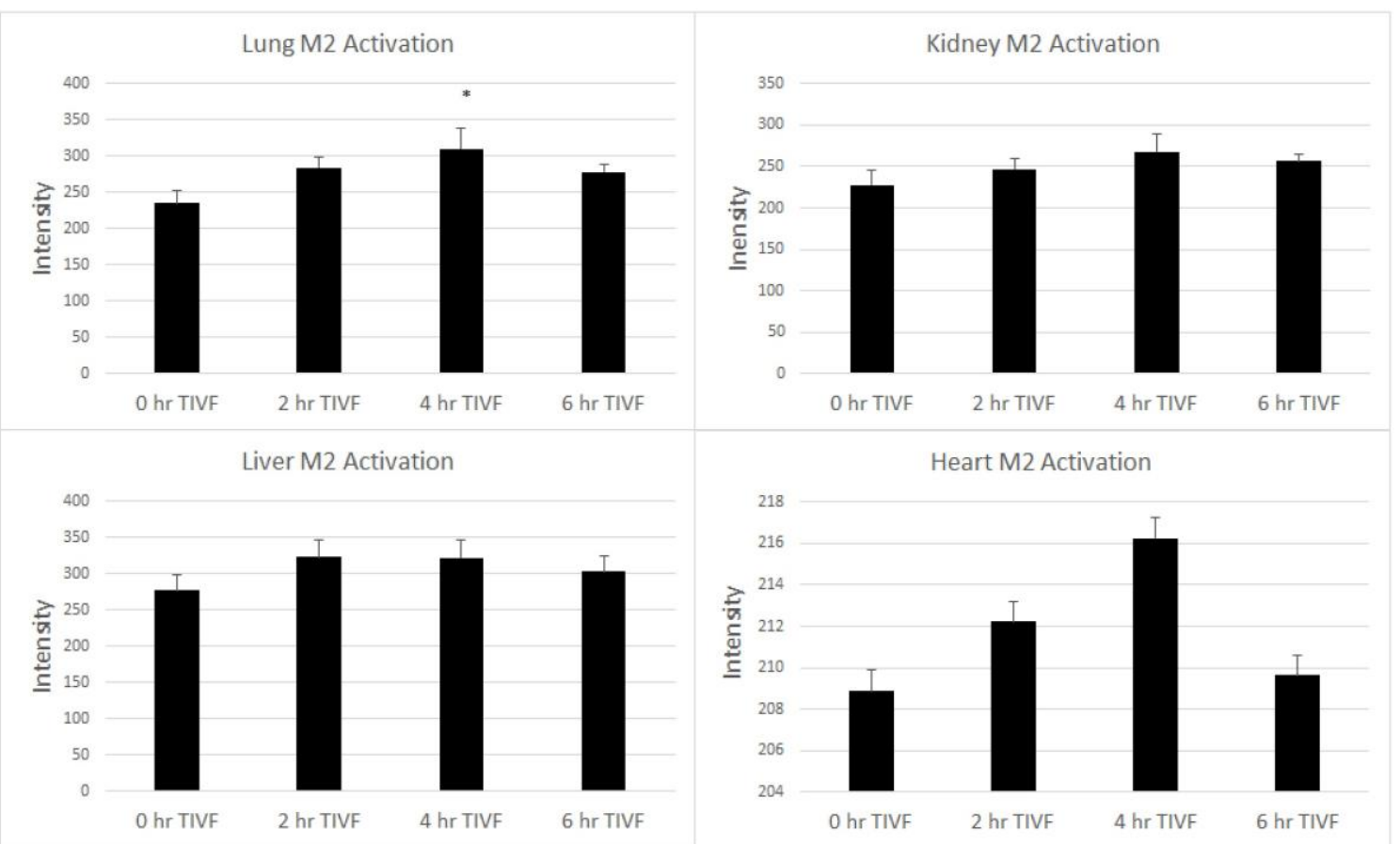

Figure 9: Intensity of M2 macrophage activation in liver, lung, kidney, and heart at 0, 2, 4 and 6 hours after brain death as measured by flow cytometry. ${ }^{*} \mathrm{p}<0.05$ vs 0 hour. $\dagger \mathrm{p}<$ 0.05 vs 2 hour. $\$ p<0.05$ v 4 hour. 

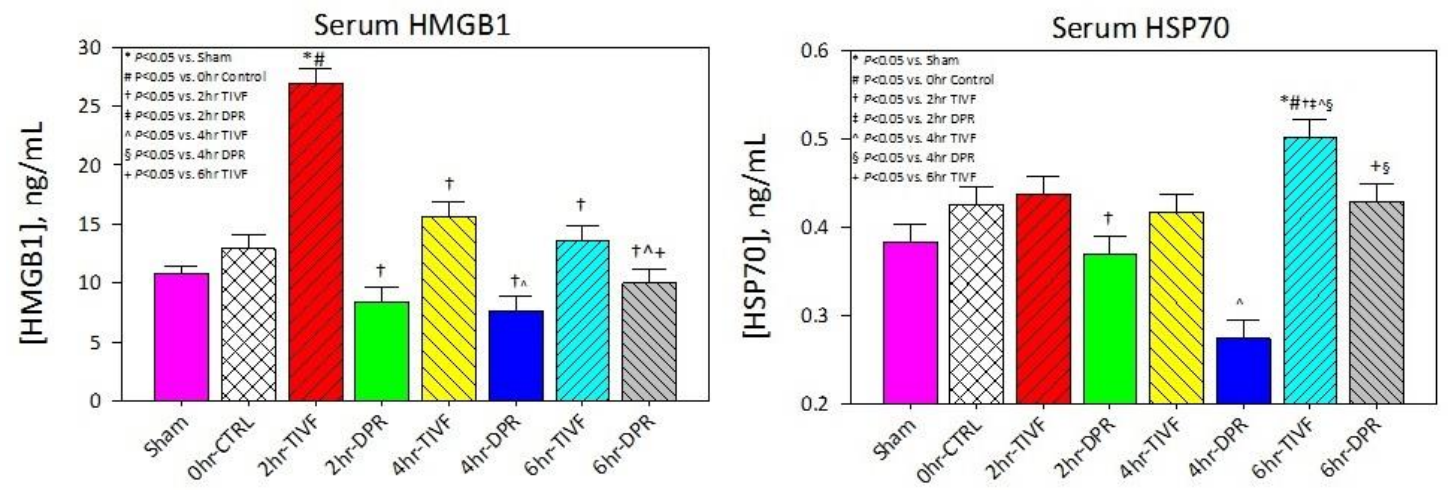

Figure 10: Serum protein levels of DAMPs High Mobility Group Box 1 Protein (HMGB1) and Heat Shock Protein 70 (HSP70) in sham rats and at 0, 2, 4, and 6 hours after brain death. ${ }^{*} \mathrm{p}<0.05 \mathrm{v}$ sham. $\# \mathrm{p}<0.05 \mathrm{v} 0 \mathrm{hr} . \dagger \mathrm{p}<0.05 \mathrm{v} 2 \mathrm{~h}$ TIVF. $\$ \mathrm{p}<0.05 \mathrm{v} 2 \mathrm{~h}$ DPR. ${ }^{\wedge} \mathrm{p}<0.05$ v 4hr TIVF. @p<0.05 v 4hr DPR. §p>0.05 v 6hr TIVF 


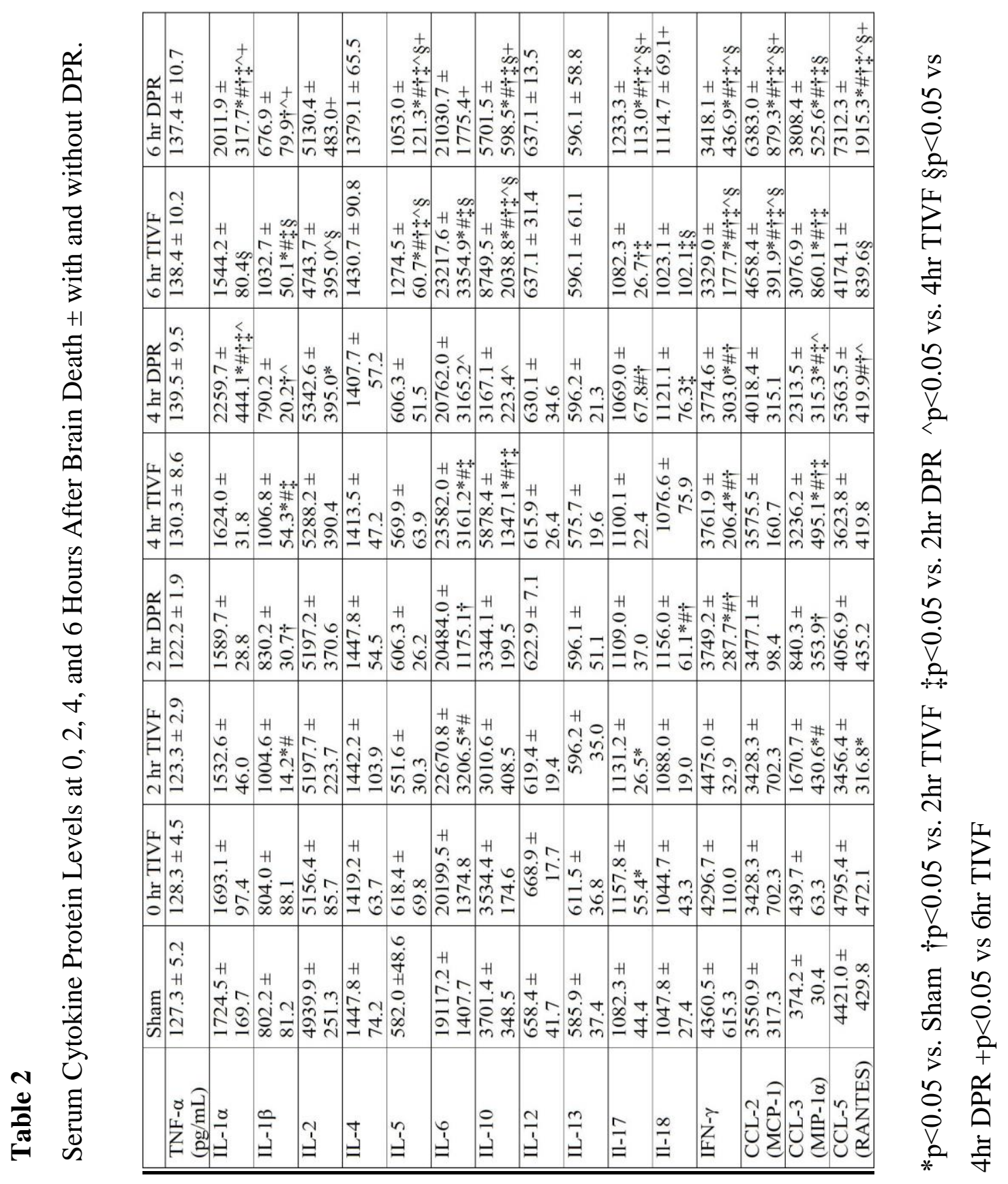


Levels of intestinal fatty acid binding protein (FABP), a measure of intestinal injury, are shown in Figure 11. Levels of FABP were increased at two, four, and six hours compared to sham, and at four and six hours compared to time zero. Use of DPR reduced FABP levels at two, four, and six hours. Lipopolysaccharide (LPS) levels were significantly increased in the TIVF groups at two, four, and six hours, while use of DPR kept LPS at sham levels at all time points. Results of immunohistochemistry staining for zona occludins (ZO-1), which is a part of tight junctions, demonstrate a breakdown in tight junctions in the ileum over time after brain death (Figure 12) which is lessened with DPR. 

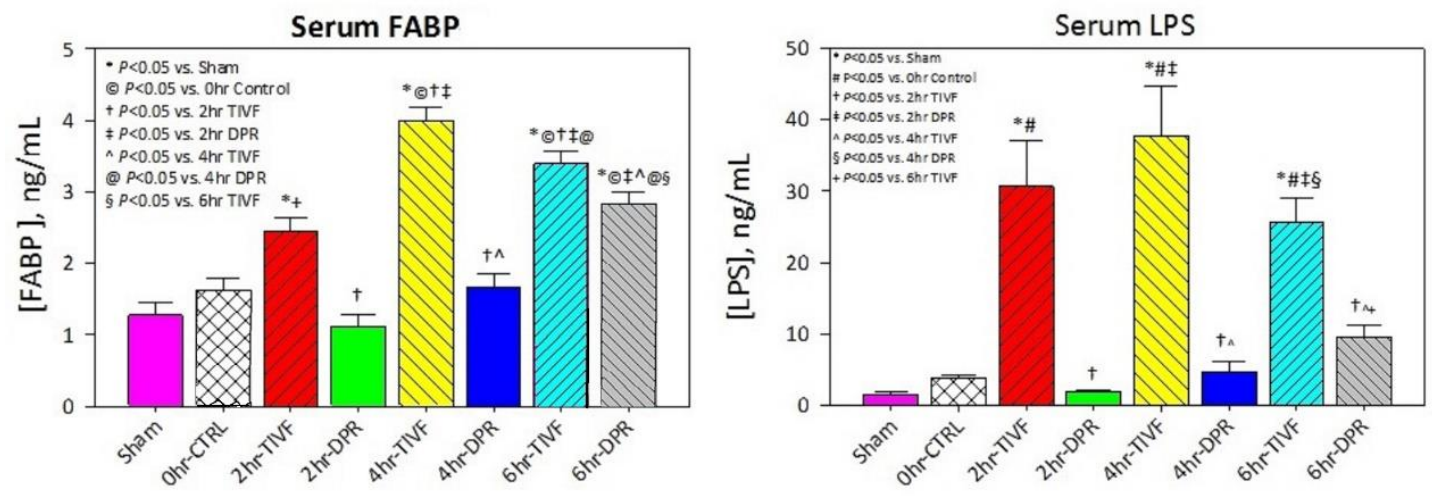

Figure 11: Serum protein levels of Fatty Acid Binding Protein (FABP) and Lipopolysaccharide (LPS) in sham rats and at 0,2, 4, and 6 hours after brain death with and without DPR. ${ }^{*} \mathrm{p}<0.05 \mathrm{v}$ sham. $\# \mathrm{p}<0.05 \mathrm{v} 0 \mathrm{hr} . \dagger \mathrm{p}<0.05 \mathrm{v} 2 \mathrm{~h}$ TIVF. $\ddagger \mathrm{p}<0.05 \mathrm{v} 2 \mathrm{~h}$ DPR. ${ }^{\wedge} \mathrm{p}<0.05$ v 4 hr TIVF. @ $\mathrm{p}<0.05$ v 4hr DPR. $\$ p>0.05$ v $6 h r$ TIVF. 


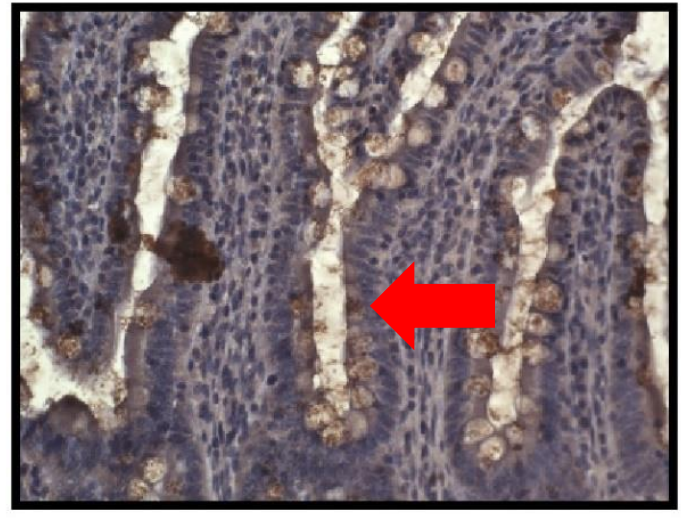

Sham

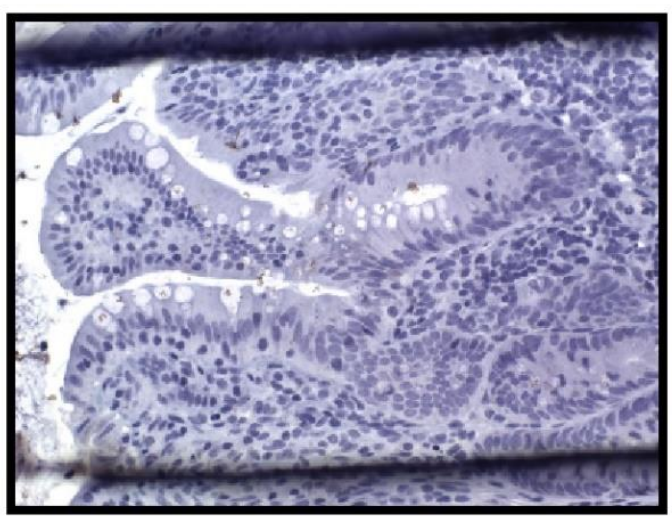

$6 \mathrm{hr}$

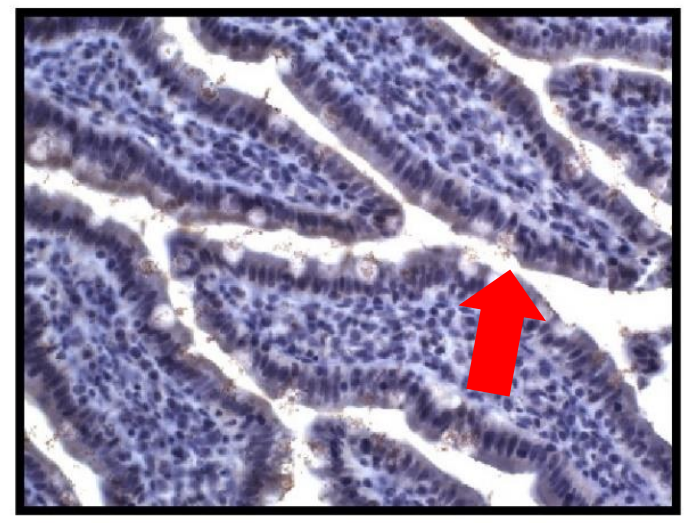

$\mathrm{O} \mathrm{hr}$

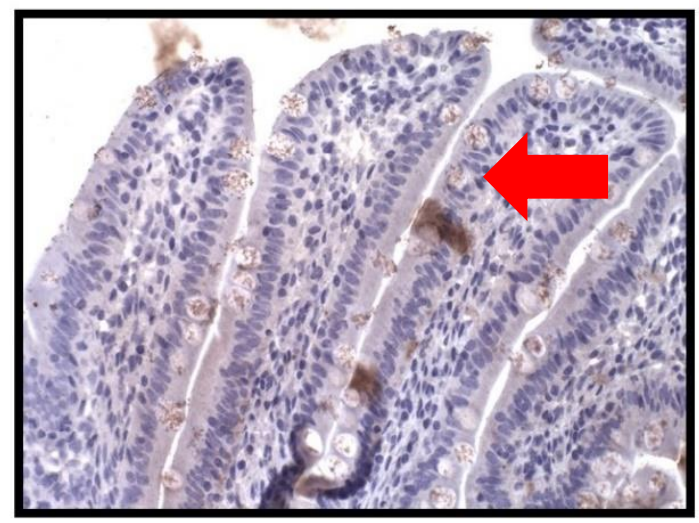

$6 \mathrm{hr}+\mathrm{DPR}$

Figure 12: Immunohistochemistry staining of tight junctions in ileum after brain death with and without DPR. Brown staining (arrows) indicates higher concentrations of ZO-1 which is part of tight junctions. 


\section{Discussion}

While the heart rate and blood pressure did not vary significantly between groups, there were significant changes in the final blood chemistry results. This can be explained by the different amount of IV fluid (IVF) the rats received. As expected, rats that were resuscitated for a longer period of time received more IVF. However, the rats resuscitated for two hours received more than half of the fluid given to the four hour rats, and almost half of that given to the six hour rats, which is consistent with the observation made during the experiments that rats required a large amount of fluid to maintain blood pressure immediately after brain death and which was then gradually tapered as the rats stabilized. Dilution from this large volume of IV fluid explains the decreases in alkaline phosphatase, amylase, total protein, and albumin seen over time in the TIVF groups. Levels of total protein and albumin were higher in all DPR groups, which required less IVF compared to their TIVF counterparts, though not all reached statistical significance. The increase is blood sodium can be explained by the fact that the rats are receiving normal saline, which has a sodium content of $154 \mathrm{mmol} / \mathrm{L}$, and over time their blood sodium moves closer to this value. Again, sodium levels in the DPR groups were decreased at two and six hours compared to TIVF alone, likely because of these animals received a smaller amount of sodium in their IV fluids. The increased blood glucose at time zero is likely due to the stress response to the sympathetic surge, as this elevated blood glucose has returned to normal by two hours. The DPR group had a higher blood glucose at two and four hours, though this only reached statistical significance at four hours. This may be because the dialysis solution contains glucose which gets absorbed into the blood stream. However, it appears that at six hours after administration, blood 
glucose levels return to normal. These laboratory results suggest that DPR helps maintain more normal, physiologic electrolyte and protein levels during resuscitation after brain death.

Despite the fact that adequate MAP was maintained, brain death did induce a significant increase in a number of inflammatory cytokines. Serum levels of TNF- $\alpha$ did not change significantly over time, though this may be due to the relatively short half-life of TNF- $\alpha$ in serum. Levels of powerful pro-inflammatory cytokines IL-1 $\beta$ and IL-6 increased in only two hours after brain death. Anti-inflammatory cytokine IL-4 was unchanged over time, while IL-10 began increasing at four hours, demonstrating that the cytokine response in brain death is not limited to the inflammatory cytokines. Cytokines IL-1 $\beta$, IL-5, IL-6, and IL-10 were decreased with DPR, demonstrating that it is able to prevent the rise of multiple pro- and anti-inflammatory cytokines. Using DPR actually caused an increase in some inflammatory mediators at different time points, such as IL$1 \alpha$, IL-2, and IL-17. This could be due to the fact that these cytokines are produced by a variety of cells, including leukocytes and endothelial cells, and DPR may affect the activity of these different types of cells differently.

This elevated inflammatory state correlates with our findings in the two DAMPs, or alarmins, HMGB-1 and HSP70. These DAMPs are part of a diverse group of molecules which are released in response to tissue injury or infection [34]. HMGB-1 is a DNA binding protein which is normally sequestered due to its chemotactic properties, but is released into the extracellular medium when tissue cells undergo necrosis. HSP70 is a member of the heat shock protein family which act as chaperones to promote proper protein folding. These proteins are also released by necrotic cells and can interact with 
toll-like receptors to induce inflammation [38]. The results show that both HMGB-1 and HSP70 increase after brain death. Use of DPR decreases serum levels of both these DAMPs. Previous authors have demonstrated that HMGB-1 and HSP70 are increased in models of intestinal ischemia $[63,64]$. As the primary function of DPR is to improve intestinal blood flow, this suggests that either these DAMPs, or their stimuli, are being released from necrotic intestinal cells, and that using DPR prevents this from happening. However this requires more study to be definitively proven.

These findings provide more direct evidence that the intestine plays a role in the inflammatory process of brain death as well. Intestinal FABP is usually found only in the intestine, and finding it in the serum indicates intestinal damage [65, 66]. Similarly, LPS is produced by bacterial within the intestine and it is released into the circulation when intestinal permeability increases in times of stress [67]. There were increases in both of these proteins in the serum after brain death. The DPR groups show significant decreases in these markers. In addition, IHC staining shows a breakdown of tight junction integrity in the ileum after brain death, further supporting the hypothesis that intestinal permeability is increased after brain death, and also demonstrates that using DPR prevents this breakdown. While a few authors have suggested the idea that the intestine plays a role in post-brain death inflammation previously $[68,69]$, it does not yet seem to have gained wide-spread acceptance in the literature. These results not only provide further evidence that the intestine is a source of this systemic inflammation, but also that DPR is able to prevent these changes in intestinal permeability, which explains how it is able to exert systemic anti-inflammatory effects. 
These inflammatory signals are associated with increased leukocyte activity. The neutrophils in tissues and blood were all increased at 6 hours, though these levels only reached statistical significance in the blood, kidney, and lung. These results also demonstrate the increase is not gradual, but rather that levels remain relatively stable for the first four hours, and then increase dramatically at six hours, especially in the kidney. The increase at six hours is consistent with multiple previous studies, including Wilhem et al. who demonstrated an increase in neutrophils in the heart at six hours after brain death [56], as well as an increase in transplanted hearts procured six hours after brain death [70], and Danobeitia and associates who showed increased neutrophils in the liver after six hours in primates [71]. However, these findings varied from other studies, including Wauters and colleagues who found that neutrophils peaked in a bronchial lavage sample at three hours in mice [72], and Schuurs and colleagues who found that neutrophil infiltration into the rat kidney began almost immediately after brain death and increased more gradually [73]. These results demonstrate that procuring organs from rats over six hours after brain death results in organs with a higher neutrophil content. Neutrophils have been implicated in the process of organ rejection [74-76]. Thus, these findings suggest that use of organs procured after this time point could result in higher rates of organ rejection, though further study would be needed to definitively prove this.

Conversely, levels of monocytes in the blood to not change significantly over time. However, within the tissues there is the same pattern of infiltration which was observed with the neutrophils, consisting of an increase at six hours that is most dramatic in the kidney. This is similar to the previously mentioned studies in the heart and lung $[56,72]$ as well as Takada et al who found increased macrophages at six hours after brain 
death in the rat kidney [15]. Jassem and colleagues demonstrated increased macrophages in livers from brain dead donors, though these were from varied time points $[77,78]$.

Macrophages in all tissues experienced an increase in M1 activation, which is typically pro-inflammatory [48]. This activation appears more gradual compared to the sudden increase in infiltration at six hours. On the other hand, M2 activation, which is typically anti-inflammatory [48], did not significant change over time. This suggests that, while the inflammatory process stimulates a more sudden and rapid influx of leukocytes at six hours after brain death, macrophage activation is stimulated shortly after brain death and gradually increases over time. In addition, like neutrophils, accumulation of macrophages has been associated with delayed graft function and increased graft rejection [79-82], and procurement of organs before this time point may result in reduced graft rejection or improved graft function, though this has not been proven.

This time of six hours for leukocyte infiltration contrasts with what has been previously been seen after hemorrhage, where this typically occurs earlier. Like previous studies in the brain death, those in hemorrhage are varied. However, the results generally show that neutrophils infiltrate tissues prior to six hours, such as those showing neutrophil infiltration in rats in less than an hour in the lung [83], by three hours in kidney [84], and the liver at four hours [85]. Similarly, macrophages have been shown to infiltrate by four hours in the lung in mice [86], and in the kidney by five hours in rats [87]. Neutrophils have been implicated as one of the earliest and primary mediators of myocardial damage after ischemia/reperfusion injury in the heart [88] and a pig model of hemorrhage has shown increased macrophage and neutrophil infiltration into the heart only 30 minutes after myocardial ischemia [89]. These findings suggest that the 
inflammation associated with brain death is not just an ischemia and reperfusion injury from labile blood pressure, but must be more complex. This could be due to hormonal changes, the changes or neural stimulation, or other factors that have not yet been determined. While more study is needed to better understand this process, what is clear is that the inflammation associated with brain death must be treated as a separate entity from that after other types of injury.

Multiple previous authors have examined specific aspects of the data presented here, including levels of specific inflammatory cytokines after brain death. However, this study looks at changes in these over time. In addition, most previous studies use mRNA to measure cytokine levels, while this one uses the more definitive protein levels. Similarly, previous studiesd typically examine only one organ, and frequently only one or a few time points. This study is the first to offer a comprehensive overview of the inflammatory processes which occur in the body after brain death in rats allowing for comparison among organs and over time.

\section{Conclusions}

In conclusion, this study offers an overall picture of the inflammatory effects of brain death in rats. Brain death is associated with an increase in inflammatory cytokines and DAMPs, and triggers leukocyte infiltration into the peripheral organs at six hours after brain death. Furthermore, it appears that the intestine becomes increasingly permeable after brain death and that this likely contributes to the inflammatory process. Using DPR helps preserve intestinal integrity and reduces systemic inflammatory mediators and has potential to improve organ inflammation. While further study is necessary to determine the applicability of these findings in humans, this represents a first 
step in understanding the complex changes that occur in brain dead donors that go on to affect the quality of the organs they donate. 


\section{CHAPTER 5}

\section{THE EFFECT OF DIRECT PERITONEAL RESUSCITATION ON LEUKOCYTE ACTIVITY IN THE LUNG}

\section{Overview}

Background: Brain death is associated with significant lung injury and inflammation. This has been associated with worse long-term outcomes for transplanted organs procured from brain death donors. Direct peritoneal resuscitation improves blood flow to the visceral organs, reduces systemic inflammation in brain death, and improves lung procurement rate. The effects of DPR on macrophage and neutrophil infiltration in the lungs is not known.

Methods: Male Sprague-Dawley rats were anesthetized and had a tracheostomy and arterial and venous cannulas placed. Brain death was achieved by inserting a 4F Fogarty catheter into the skull and slowly inflating the balloon. Rats were resuscitated with normal saline to maintain a mean arterial pressure of $80 \mathrm{mmHg}$ (targeted intravenous fluid, TIVF) and DPR animals also received an intraperitoneal injection of commercial peritoneal dialysis solution. Rats were sacrificed at zero, two, four, and six hours after brain death and tissue samples taken. Protein levels were assessed using quantitative ELISA. Flow cytometry and immunohistochemistry were used to quantify neutrophil and macrophage infiltration.

$\underline{\text { Results: }}$ At all time points, use of DPR downregulated multiple inflammatory cytokines in the lung including IFN- $\gamma, \mathrm{TNF}-\alpha$, IL-1 $\alpha$, and IL-18. At four and six hours post-brain 
death, adhesion molecules intracellular adhesion molecule, E-selectin, and P-selectin were increased above sham. At each of these time points use of DPR caused a significant decrease in protein expression. A similar pattern was seen in vascular adhesion molecule at two and six hours. Infiltration of macrophages and neutrophils was substantially decreased with DPR on immunohistochemistry straining and trended down at six hours with DPR, though this difference was not statistically significant on flow cytometry. Macrophage activation was unchanged by DPR.

Conclusions: Animals which received TIVF alone had significant increases in inflammatory cytokines within the lung tissue, leading to adhesion molecule expression and ultimately in leukocyte infiltration. All of these stages of inflammation were downregulated by use of DPR. Using DPR in brain dead organ donors shows promise as a way to reduce lung injury and inflammation. 


\section{Background:}

Brain death is associated with significant lung injury. In addition to the contusion and aspiration that frequently accompany the initial injury, brain dead patients frequently develop neurogenic pulmonary edema [6]. This edema is theorized to originate from two possible sources: hypertension causing increased capillary pressure and direct pulmonary capillary damage [90], or increased capillary permeability due to $\alpha$-adrenergic stimulation [91]. Brain death also causes significant systemic inflammation [16] as well as inflammation and apoptosis within the lung tissue itself [92-94]. When these lungs are procured for transplantation, this inflammation leads to worse ischemia-reperfusion injury [95] and higher rates of rejection [96]. This lung damage can be worsened by resuscitation after brain death. Large amounts of intravenous (IV) crystalloid fluid is often given to support blood pressure, but this can worsen lung edema [7]. In part because of this inflammation and edema, only $20 \%$ of lungs from brain dead donors are considered suitable for transplantation [57].

Because of these problems with conventional IVF resuscitation, DPR has potential to improve organ donor resuscitation. Previous studies have demonstrated that use of DPR in a rat model of brain death leads to improved visceral organ blood flow, reduced organ edema, and reduced serum levels of multiple inflammatory cytokines [41]. Using of DPR in brain dead human organ donors improved the rate of organ procured per donor, specifically by increasing rates of lung procurement [27]. The purpose of this study is to determine whether the anti-inflammatory effects of DPR would lead to a reduction in the infiltration of macrophages and neutrophils into the lung after brain death. 


\section{Methods}

The methods were performed as outlined in Chapter 2: Materials and Methods. In brief, our murine model of brain death was used and rats were sacrificed immediately after brain death (time zero) as well as two, four, and six hours after brain death. Those scheduled for DPR received a single intraperitoneal injection of $30 \mathrm{~mL}$ of Delflex solution. After brain death rats were resuscitated with enough normal saline to maintain a MAP of $80 \mathrm{mmHg}$ and vitals were measured continuously. After sacrifice, lung tissue was collected and flash frozen in liquid nitrogen and ultimately used for protein analysis with ELISA, digested in collagenase for creation of a single cell suspension for flow cytometry, or preserved in neutral buffered formalin for immunohistochemistry staining.

\section{Results:}

Levels of cytokines and chemokines in the lung tissue are displayed in Table 3. Multiple pro-inflammatory cytokines, including IFN- $\gamma$, TNF- $\alpha$, IL-1 $\alpha$, IL-1 $\beta$, and IL-6 were increased after brain death and decreased with the addition of DPR. Antiinflammatory mediators IL-4 and IL-10 were also increased at two, four, and six hours compared to sham and decreased with DPR. Levels of IL-12 peaked at four hours and were decreased by DPR at that time, while levels of IL-2 did not increase until six hours after brain death and were further upregulated with the addition of DPR. 


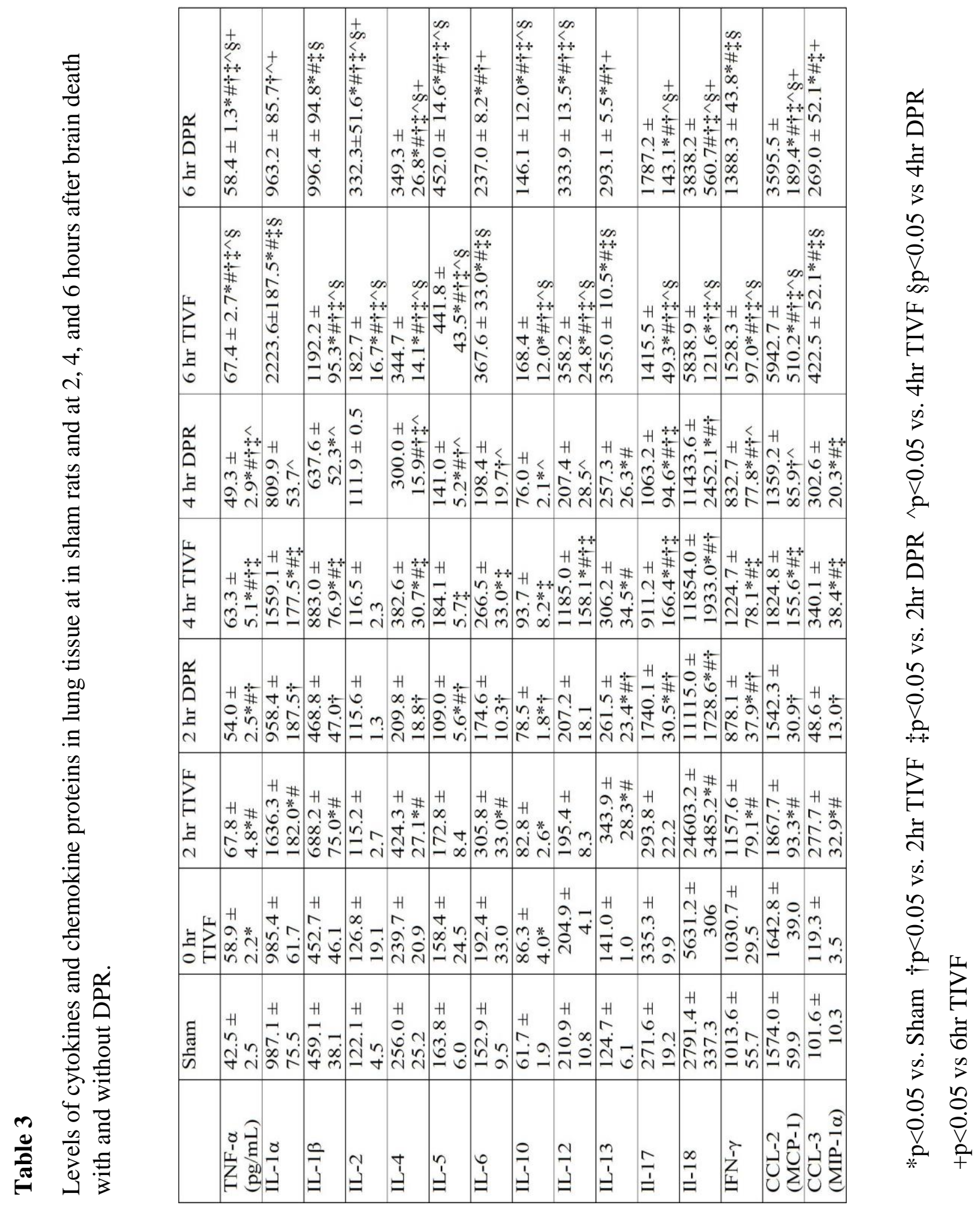


Adhesion molecules intracellular adhesion molecule (ICAM), vascular adhesion molecule (VCAM), E-selectin, and P-selectin are shown in figures 13-16. Levels of ICAM, E-selectin, and P-selectin remained equivalent to sham levels at two hours, then increased at four hours and six hours in the TIVF groups, and were decreased with DPR compared to TIVF at the same time point. Protein levels of VCAM were increased at two and six hours compared to sham and decreased at both time points with DPR. Levels of VCAM were equivalent to sham at four hours with and without DPR.

Results of the flow cytometry analysis for neutrophils are shown in figure 17 . In the TIVF group there is a large increase in the number of neutrophils that infiltrate the tissue at six hours. Use of DPR reduced the size of this increase, though the difference between these two points failed to reach statistical significance. Neutrophil activation measured by myeloperoxidase (MPO) activity is shown in figure 18. The MPO activity increased immediately after brain death (time zero) compared to sham. The MPO activity further increased at six hours. Use of DPR reduced MPO activity at two, four, and six hours compared to the TIVF group at each respective time point.

Macrophage infiltration, measured by flow cytometry, increased at six hours, and this increase was dampened by DPR, though this was not a statistically-significant change (Figure 19). Use of DPR did not change M1 activation, and decreased M2 activation at four hours compared to the four hour TIVF group, but the TIVF and DPR groups were equivalent by six hours (Figure 20). 


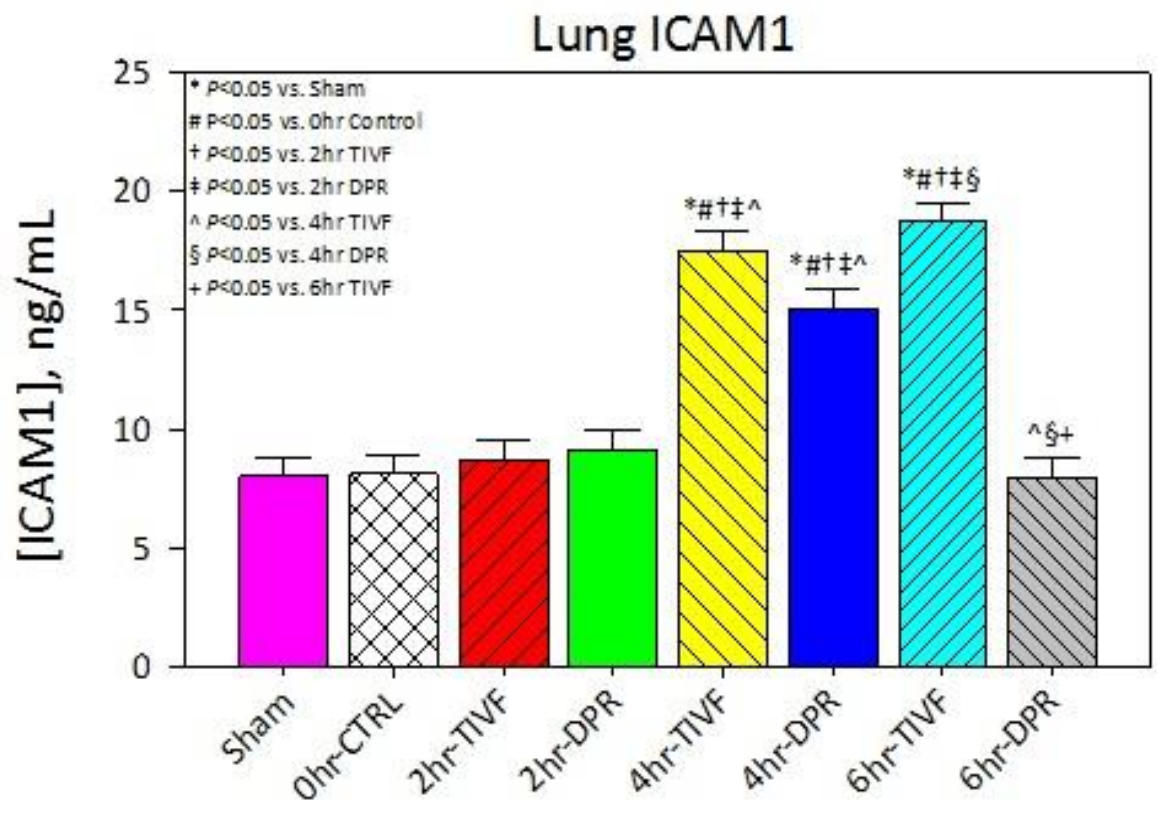

Figure 13: Levels of ICAM protein in lung tissue in sham rats and at 0,2, 4, and 6 hours after brain death with and without DPR. 


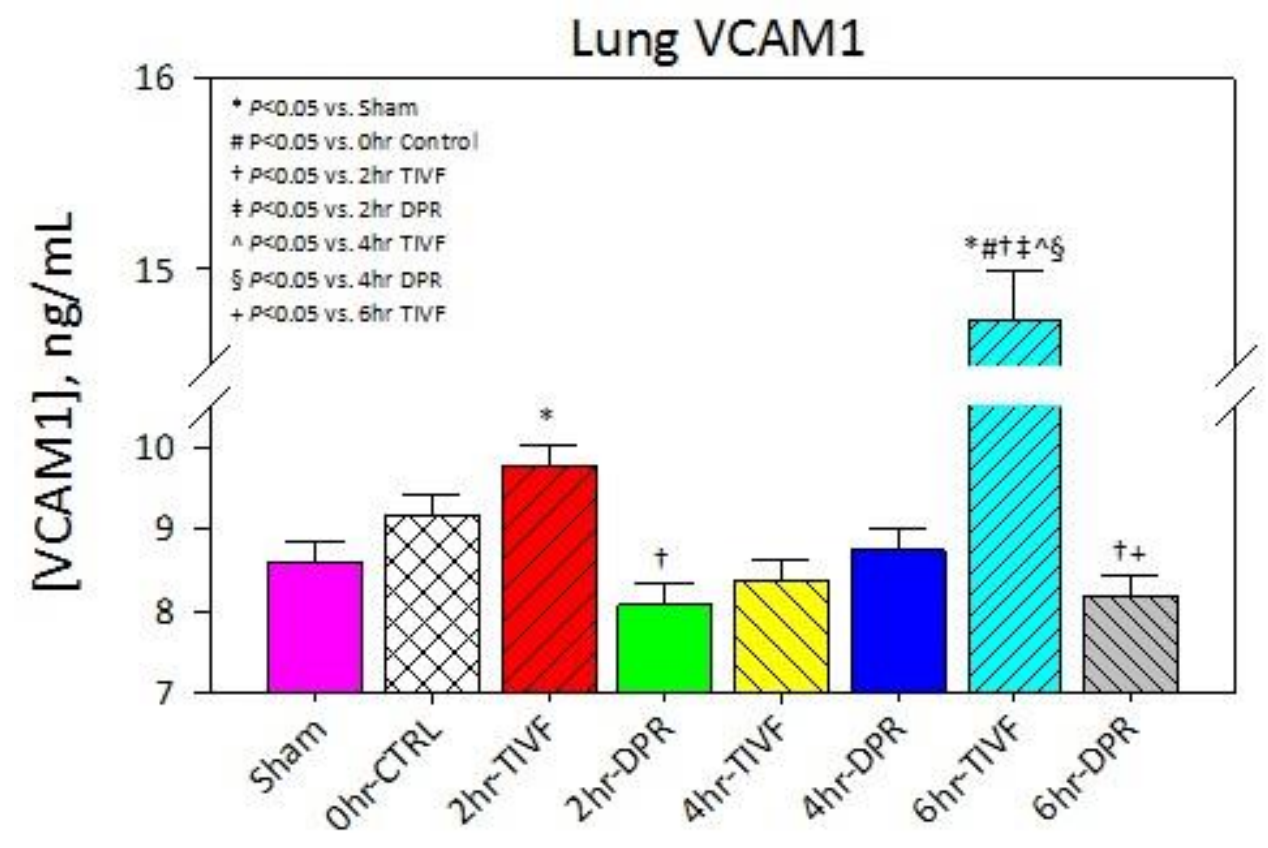

Figure 14: Levels of VCAM protein in lung tissue in sham rats and at 0, 2, 4, and 6 hours after brain death with and without DPR. 


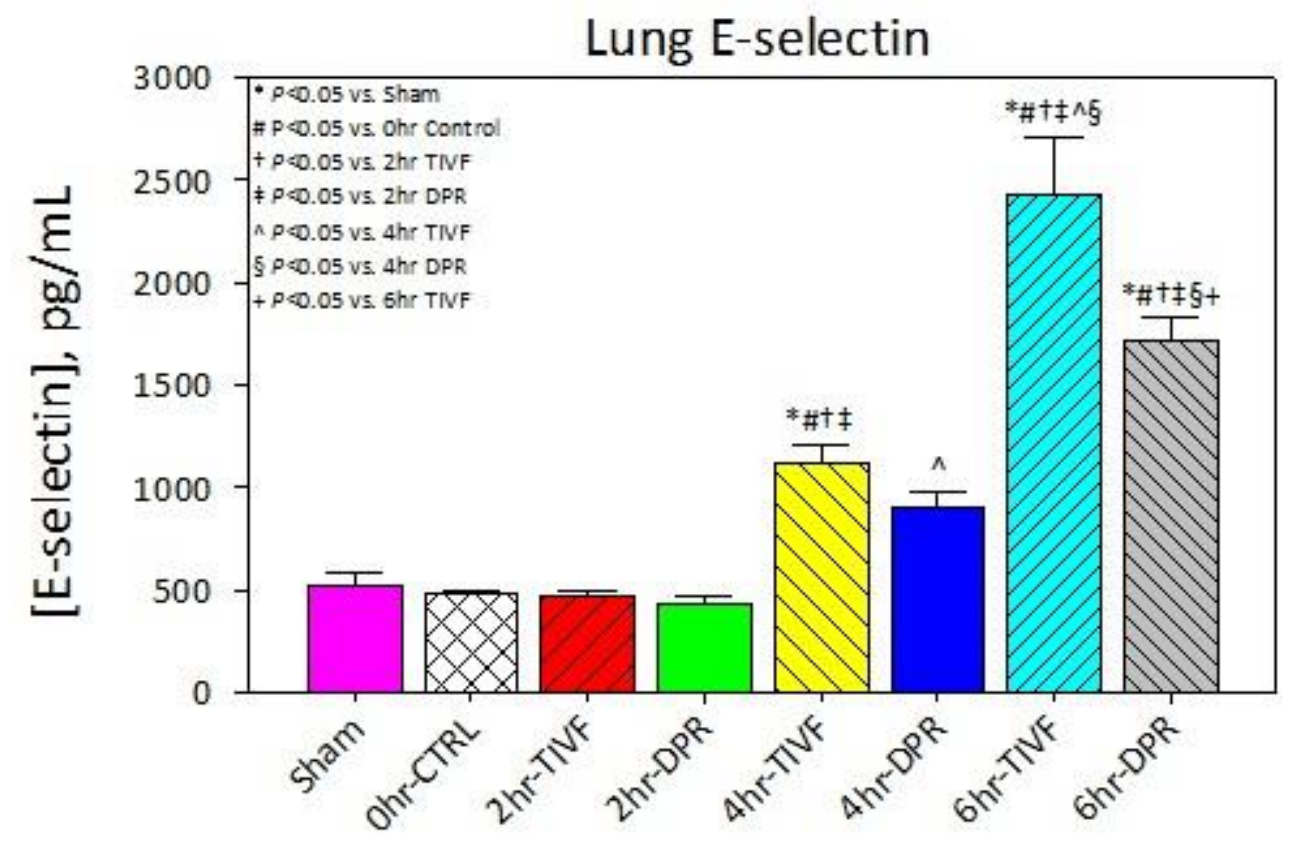

Figure 15: Levels of E-selectin protein in lung tissue in sham rats and at 0,2, 4, and 6 hours after brain death with and without DPR. 


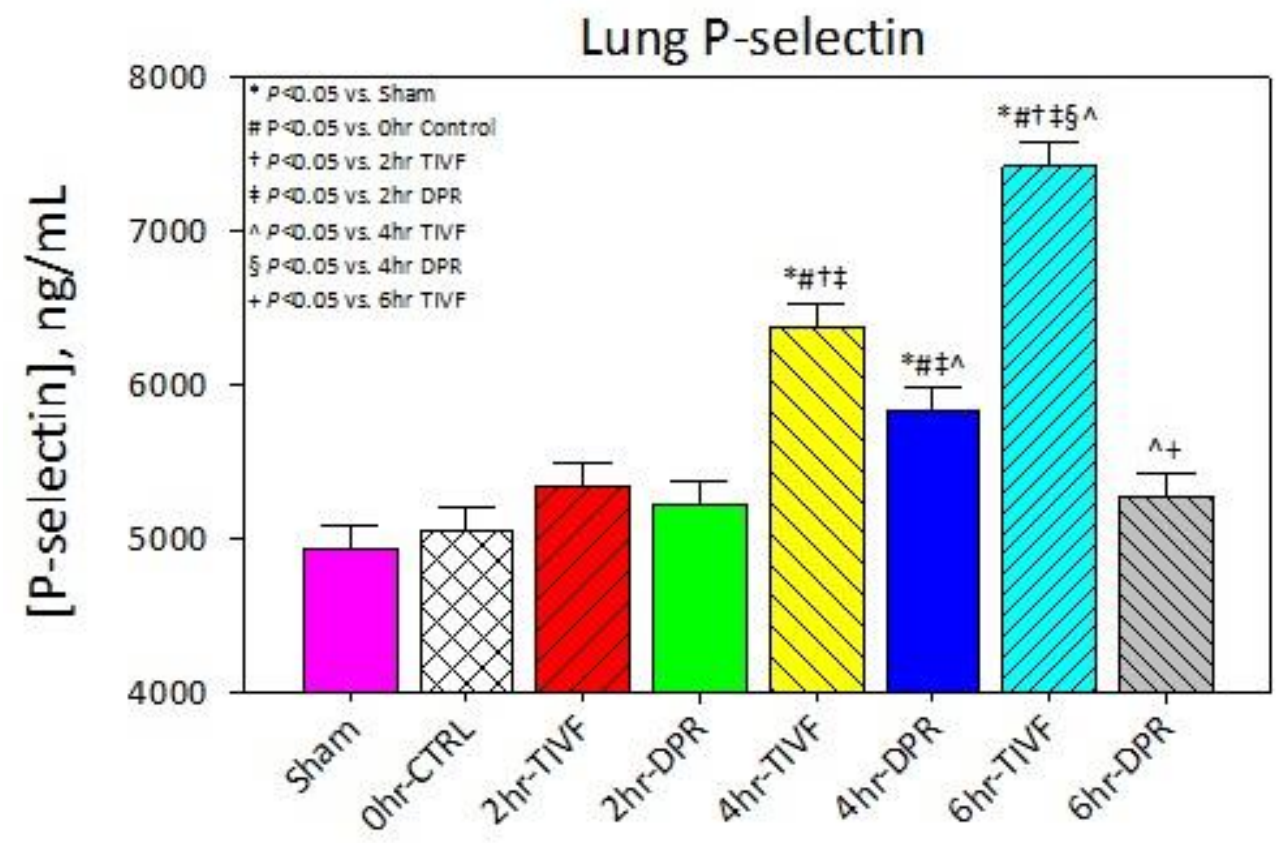

Figure 16: Levels of P-selectin protein in lung tissue in sham rats and at 0, 2, 4, and 6 hours after brain death with and without DPR. 


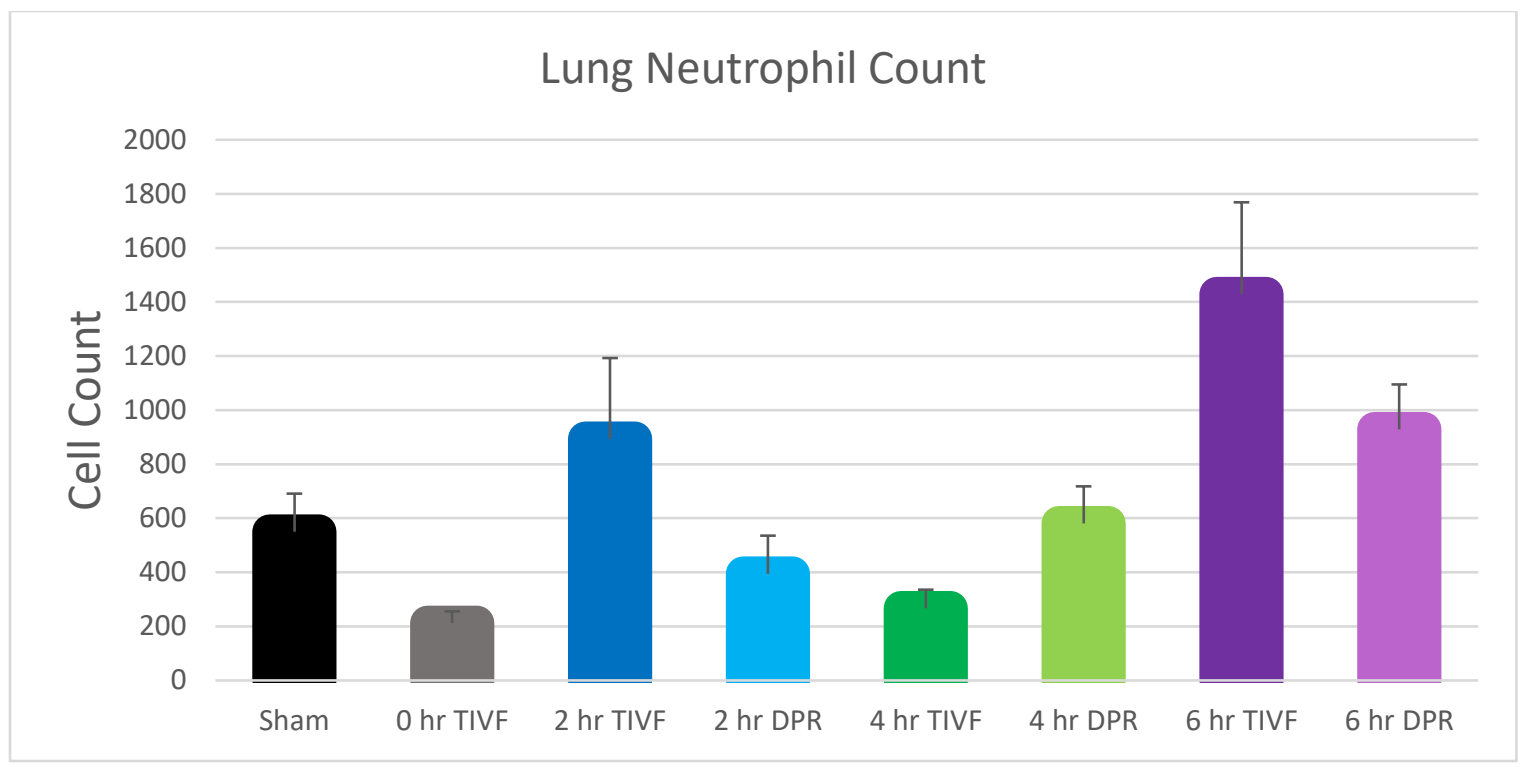

Figure 17: Flow cytometry results of neutrophils within the lung in sham rats and at 0,2, 4, and 6 hours after brain death with and without DPR. 


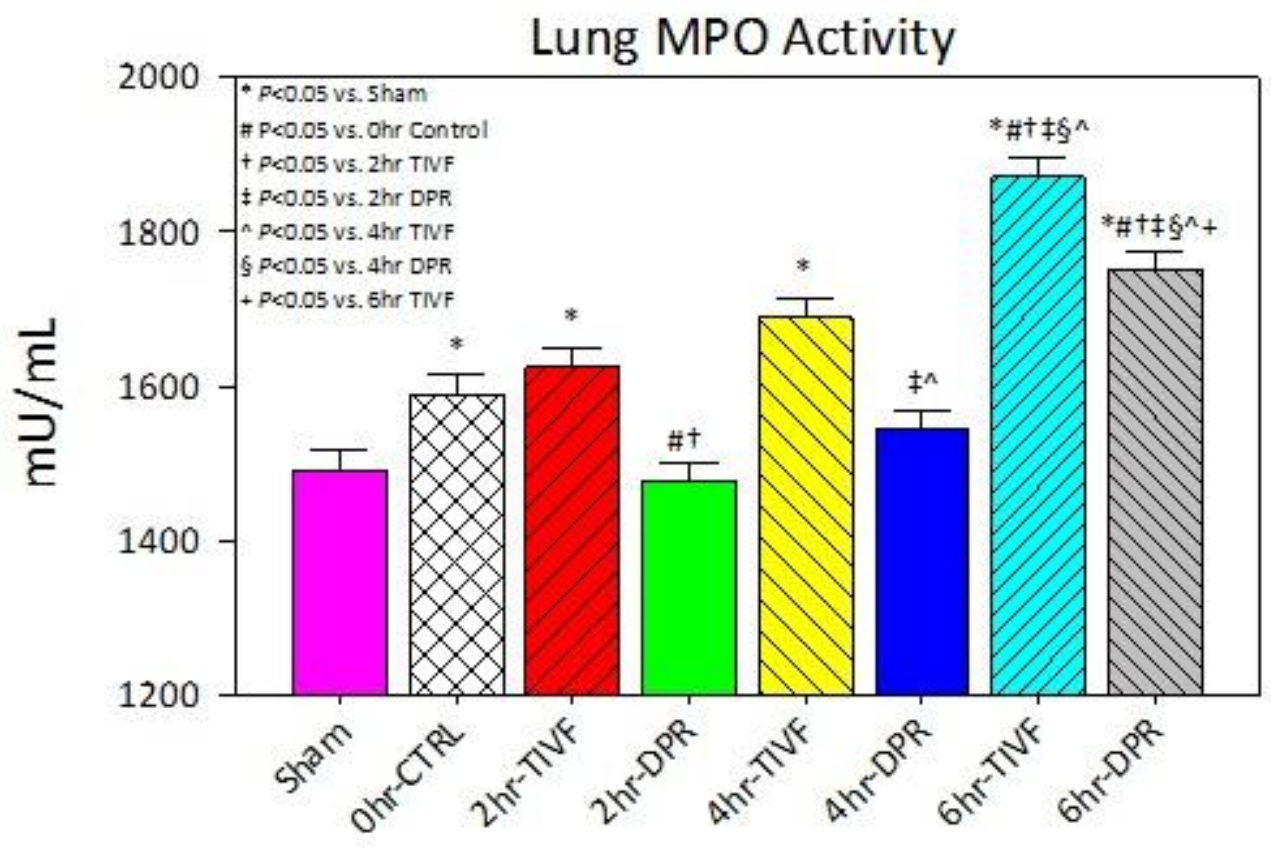

Figure 18: Myeloperixodase activity assay within the lung in sham rats and at 0,2, 4, and 6 hours after brain death with and without DPR 


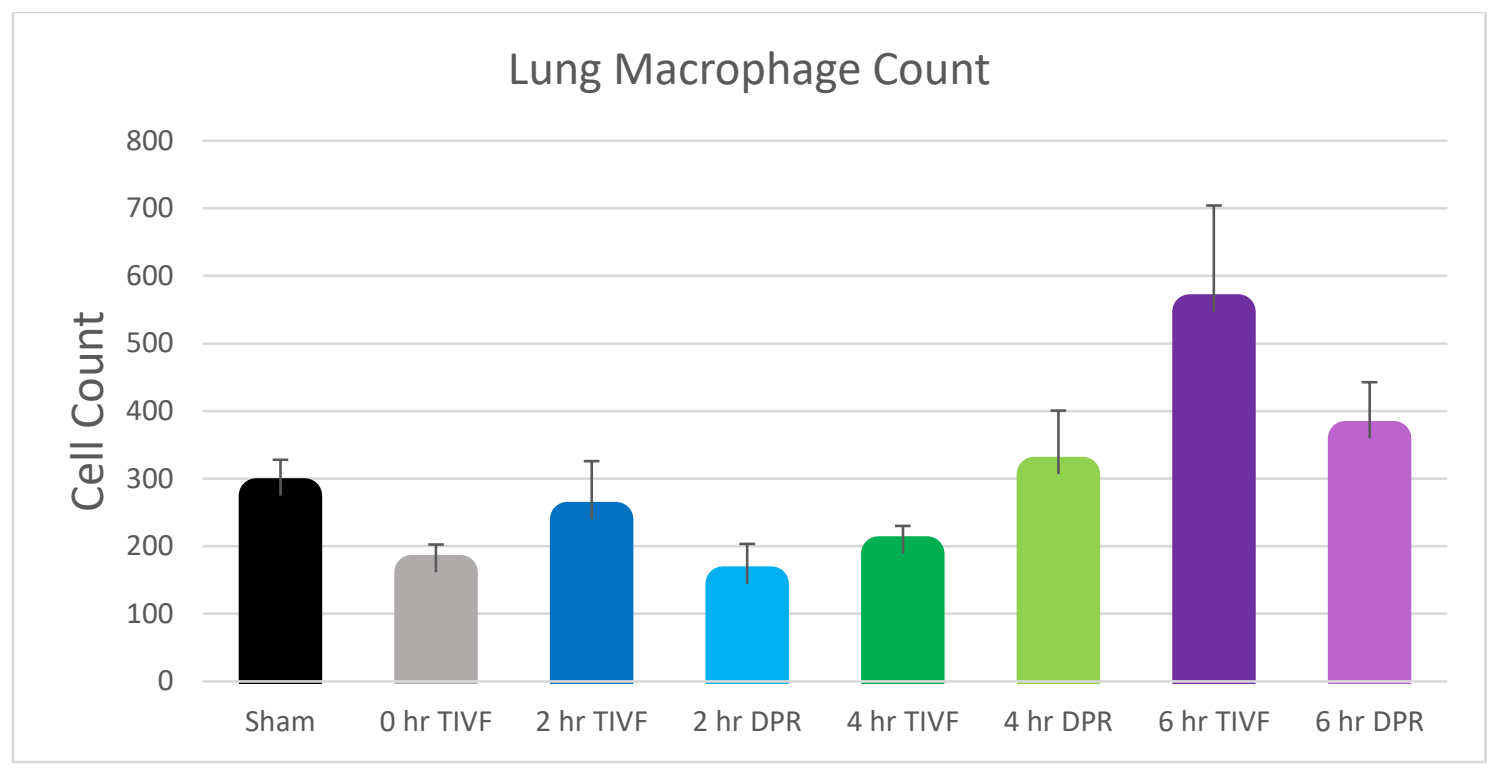

Figure 19: Flow cytometry results showing macrophage infiltration in the lung in sham rats and at $0,2,4$, and 6 hours after brain death with and without DPR 

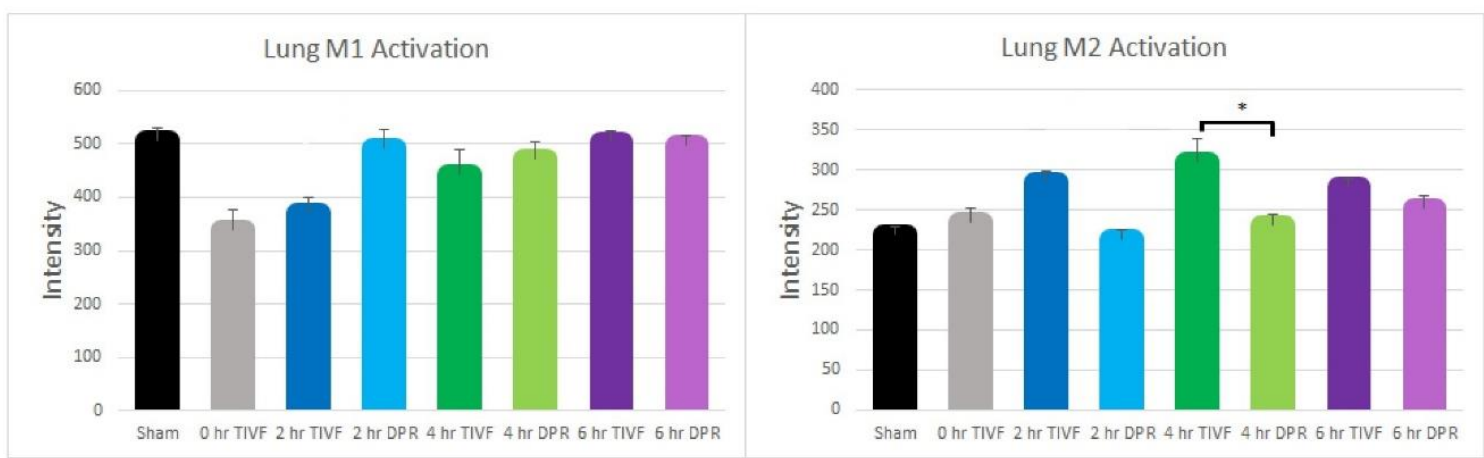

Figure 20: Flow cytometry results showing M1 and M2 macrophage activation in the lung in sham rats and at $0,2,4$, and 6 hours after brain death with and without DPR. *p < 0.05 vs DPR group at same time point. 
Immunohistochemistry staining reflected the increase in macrophages and neutrophils in the tissue at six hours compared to time zero and the slight decrease with DPR (Figures 21 and 22). Pathologist descriptions (Table 4) show that use of DPR at six hours significantly improved lung edema and hemorrhage and mildly reduced leukocyte infiltration. Compared to sham, the process of brain death itself caused some edema, marked atelectasis, and presence of some macrophages and neutrophils in the alveolar spaces. 


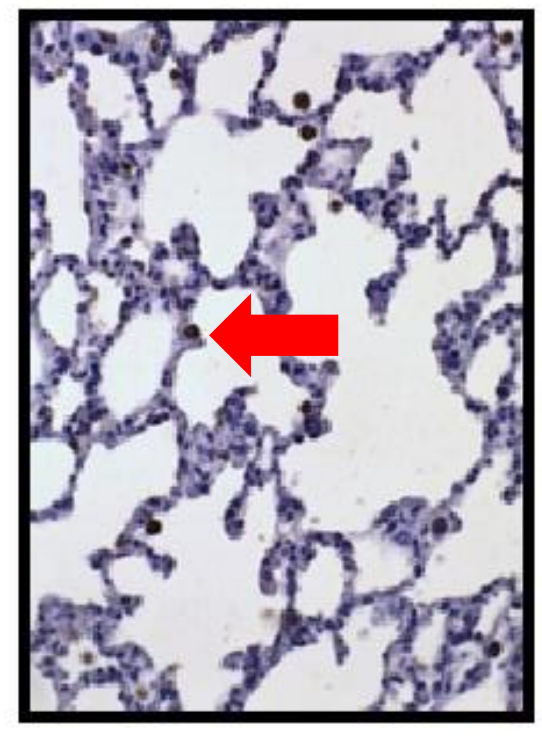

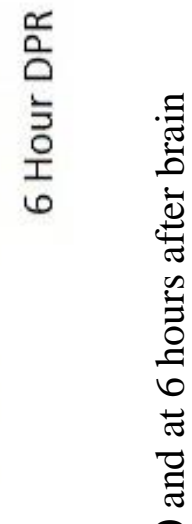

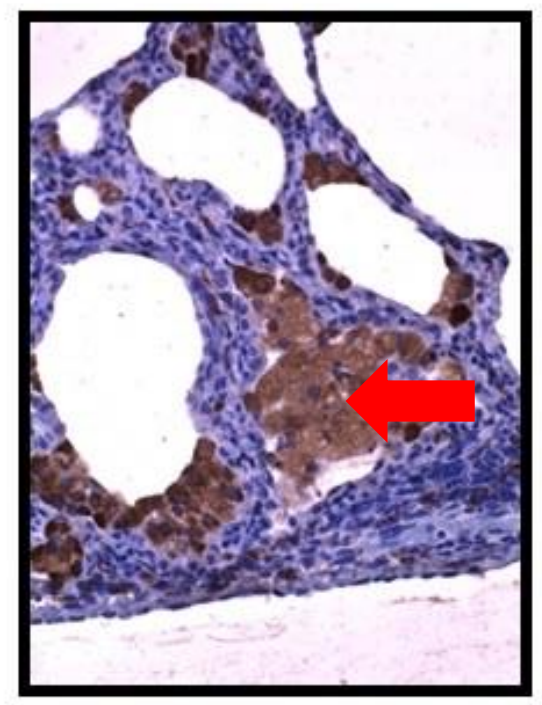

.

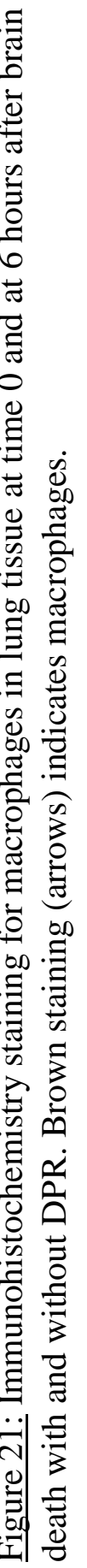

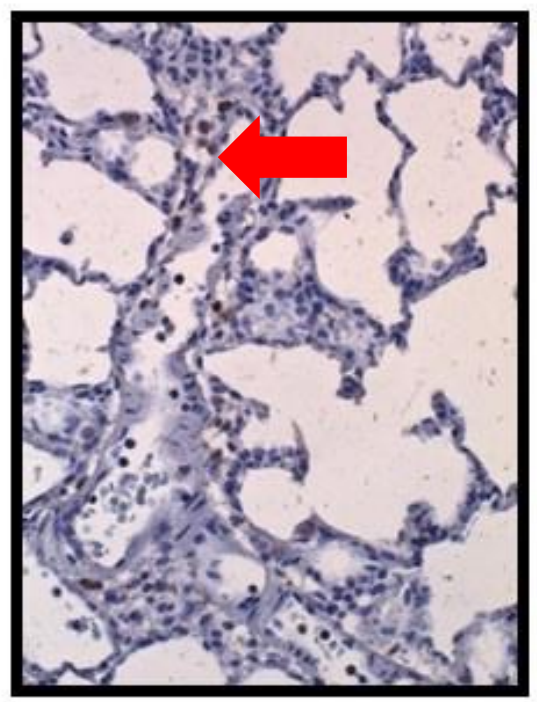

\begin{tabular}{l}
\hline \\
$\geqq$ \\
$\vdots$ \\
$\frac{1}{3}$ \\
$\frac{1}{6}$
\end{tabular}

$+\quad \quad \Xi \Xi$

E..

히엉

( ) 융 


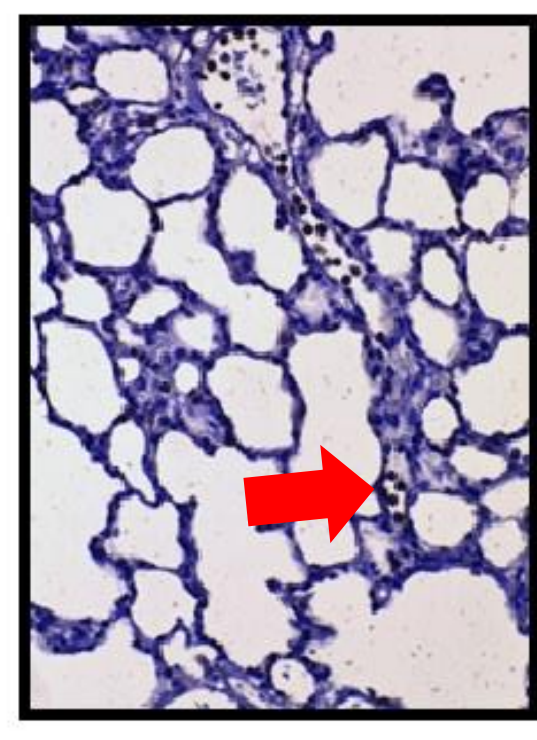

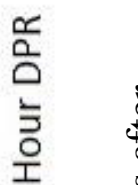

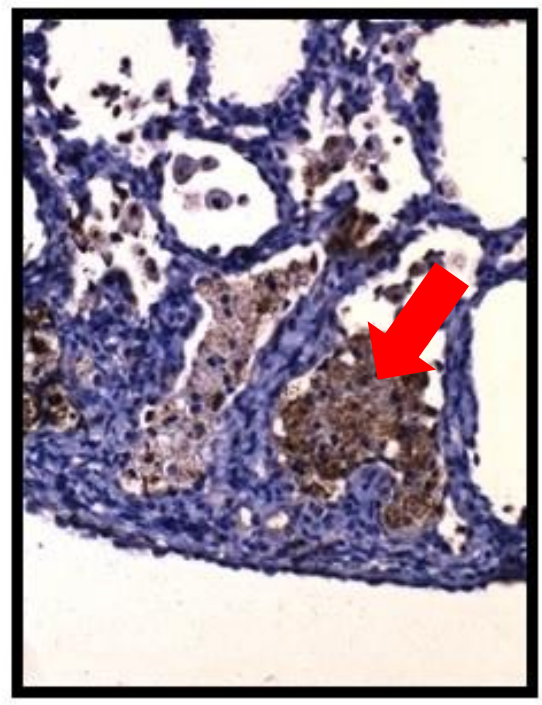

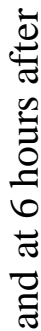

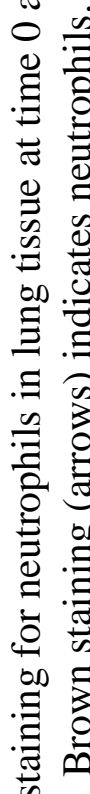

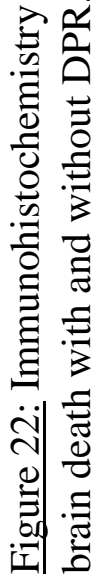

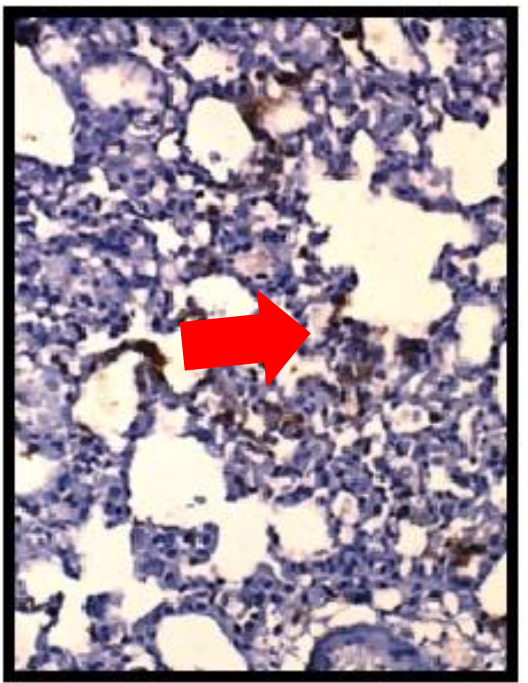

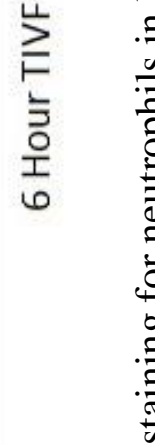

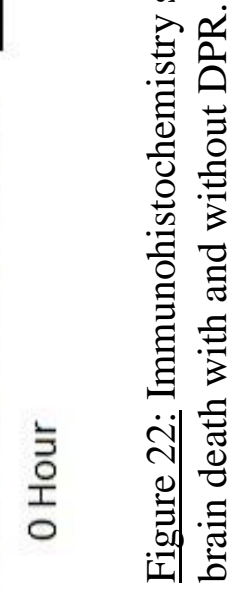




\section{Table 4}

Pathology descriptions of lung tissue in sham rats and at time zero, six hours, and six hours with DPR

\begin{tabular}{|l|l|l|l|l|}
\hline & Sham & 0 hour & 6 hour & 6 hour DPR \\
\hline Edema & Absent & Mild-moderate & Moderate-Severe & Mild-moderate \\
\hline Hemorrhage & Absent & Absent & Moderate-Severe & Focal \\
\hline $\begin{array}{l}\text { Quantity of } \\
\text { Neutrophils }\end{array}$ & None & Few & Numerous & Numerous \\
\hline $\begin{array}{l}\text { Location of } \\
\text { Neutrophils }\end{array}$ & None & $\begin{array}{l}\text { Alveolar wall, } \\
\text { intravascular }\end{array}$ & $\begin{array}{l}\text { Intraalveolar } \\
\text { spaces }\end{array}$ & Alveolar wall \\
\hline $\begin{array}{l}\text { Quantity of } \\
\text { Macrophages }\end{array}$ & Few & Few & Numerous & Few \\
\hline $\begin{array}{l}\text { Location of } \\
\text { Macrophages }\end{array}$ & $\begin{array}{l}\text { Alveolar } \\
\text { wall }\end{array}$ & $\begin{array}{l}\text { Alveolar wall, } \\
\text { intravascular }\end{array}$ & $\begin{array}{l}\text { Intraalveolar } \\
\text { spaces }\end{array}$ & $\begin{array}{l}\text { Intraalveolar } \\
\text { spaces }\end{array}$ \\
\hline
\end{tabular}




\section{Discussion:}

The inflammation following brain death, or any inflammatory stimulus, requires leukocytes to move out of the blood stream and into the tissues. This requires a complex series of steps including adhering to the blood vessel wall, rolling along the wall, and traversing the vessel wall into the tissue. This process depends on the interaction of numerous proteins on the cells and on the vessel wall. P-selectin is expressed by the endothelium to initially attract and adhere leukocytes, while E-selectin is expressed by activated endothelium to slow the adherent leukocytes and help them roll [97]. Similarly, ICAM and VCAM are expressed by the endothelium to bind ligands on the leukocytes to cause them to adhere to the vessel wall in preparation for transmigration $[97,98]$. Thus, targeting these molecules is a method of reducing leukocyte infiltration into tissues in inflammation [99].

These data help delineate the timing of events that lead to this leukocyte infiltration and lung injury after brain death. The process of brain death and resuscitation appears to cause at least a temporary increase in all cytokines, both pro- and antiinflammatory, as there were increases in IL-4 and IL-10, as well as pro-inflammatory cytokines IL-1 $\alpha$, IL-1 $\beta$, IL-6, IL-18, IFN- $\gamma$ and TNF- $\alpha$. However, the pathology staining makes it clear that the overall effect is pro-inflammatory, associated with edema, hemorrhage, and lung injury. While the timing of individual cytokines may vary, a number of vital pro-inflammatory cytokines, such as IFN- $\gamma$, IL-1 $\alpha$, and IL-1 $\beta$ were increased at two hours, and TNF- $\alpha$ was increased immediately after brain death, suggesting that these changes are likely some of the first in the complex cascade of 
events which follow brain death. While inflammation may begin as a systemic process, it induces increased inflammatory mediators within the lung tissue itself.

Several of the elevated inflammatory cytokines, including IL-1 and TNF- $\alpha$, also cause the expression of leukocyte adhesion molecules [100]. In addition, these results show that tissue levels of CCL-2, an attractant for macrophages, increased significantly after two hours though most dramatically at six hours, while CCL-5, a chemokine primarily for T-cells, peaks at two hours and then gradually decreases. These findings demonstrate that at two hours after brain death, the lung tissue is preparing for an influx of leukocytes. Protein levels of leukocyte adhesion molecules then become increased in the lung at four hours after brain death. This then leads to infiltration of neutrophils and macrophages into the lung tissue between four and six hours after brain death. This is consistent with previous studies that have found significant leukocyte infiltration in the lungs at six hours [72]. However, these data demonstrate that instead of a gradual increase, this is a rapid increase that occurs between four and six hours after brain death in the rat.

These findings vary from those found in other types of shock. After hemorrhagic shock several authors have found increased neutrophil infiltration and ICAM expression increased three hours after hemorrhage in rats and mice $[83,101]$. Other authors have examined lungs after ischemia and reperfusion and injury and found increased macrophages, neutrophils, and ICAM after two to four hours in rats [102, 103]. Sheridan et al. found increased lung neutrophil accumulation after four hours in a rat model of sepsis [104]. While these studies are variable, they all suggest that, compared to the data in this study, macrophages and neutrophils infiltrate into the lungs earlier in other types 
of injury when compared with brain death. This could be due to a number of factors that make brain death different from other types of injury, such as the neural and hormonal changes. In addition, previous studies have shown that inflammation increased both with hemorrhage and resuscitation $[83,105]$, so it is also possible that the resuscitation after brain death, which relies more heavily on large volumes of crystalloid solution, may be an additional source of these differences.

Use of DPR affects each stage in the process of leukocyte infiltration. While the results were not statistically significant, the flow cytometry suggests that use of DPR blunts the increase in macrophage and neutrophils seen in animals which received TIVF alone. This is likely due to the fact that DPR causes a downregulation of adhesion molecules. Even though macrophages are activated just as strongly when DPR is given, this reduced expression of adhesion molecules prevents their moving into the lung tissue as easily. The adhesion molecule expression is, in turn, determined by the inflammatory cytokines, especially IL-1 and TNF- $\alpha$. These are the primary stimuli for adhesion molecule upregulation, and were also downregulated with DPR. Other cytokines that are markers of inflammation, such as IL-6, IL-12, and IFN- $\gamma$ were also downregulated with DPR, suggesting that the lungs are in an overall less inflammatory state. However, the change in cytokines presents a dilemma regarding cause and effect, as these cytokines stimulate the adhesion molecules which promote leukocyte infiltration, and also promote macrophage and neutrophil activation, but traditionally macrophages and neutrophils are one of the primary sources of inflammatory cytokines. This suggests that inflammatory stimulation in brain death must be coming from other sources as well. 
The cause of the systemic inflammation associated with brain death is a source of debate. It has been suggested that the process of brain death is associated with damage to the blood-brain barrier, and that inflammatory mediators are released from the brain itself $[16,17]$ or from endothelial damage [91]. Other authors have suggested that some of this inflammation arises from the intestine $[69,106]$, and the data presented in chapter four supports this. This is consistent with the demonstrated increases in intestinal permeability in other types of shock $[12,14,107]$. Furthermore, it has been shown that renal ischemia and reperfusion can lead to changes in neutrophil margination within the lung, demonstrating that the lungs can suffer injury from an insult to an entirely different organ [108]. The fact that changes within the intestine can affect the lungs explains how DPR, a therapy applied in the abdomen and that targets intestinal blood flow, is able to reduce systemic inflammation and produce changes within the lungs.

While DPR appears to affect the mechanism for leukocyte infiltration, it does not reduce leukocyte activation. While there are some small differences in M2 macrophage activation at four hours after brain death, the TIVF and DPR groups are equivalent by six hours. The MPO activity of neutrophils was reduced by DPR at each time point, though since this measures the overall activity within the tissues it cannot be determined from this data whether this is due to changes in the activity of the individual neutrophils, or due to a reduction in the number of them present in the tissue, or both. This suggests that, while the upregulation of leukocyte adhesion molecules can be altered by the resuscitation process, the leukocyte activation is triggered by the process of brain death itself and cannot be altered this way. This is consistent with our results that compare sham and time zero animals; while no changes in the leukocyte adhesion molecules 
occurs in this time frame, there is an increase in MPO activity. In addition, there were small but notable increases in leukocytes, inflammation, and edema in the lung tissue at time zero compared to sham animals, even though they received less total IV fluid.

Clearly the process of brain death itself, and not just its subsequent labile blood pressures and resuscitation, leads to inflammation and edema within the lungs. While using DPR is not sufficient to prevent all of these changes, it does help reduce edema, inflammation, and leukocyte infiltration, explaining how using DPR helps increase the number of lungs suitable for transplantation.

\section{Conclusions}

In conclusion, our data suggest that by maintaining the blood flow to the intestine during brain death, this decreases the permeability of the intestine, thus preventing the release of inflammatory mediators. This, in turn, leads to improvements in inflammatory signals within the lung tissue, which decreases the upregulation of leukocyte adhesion molecules and ultimately reduces the infiltration of macrophages and neutrophils. While further study is needed to confirm the causative nature of these findings, use of DPR shows great potential as a way to reduce lung injury and inflammation after brain death. 


\section{CHAPTER 6}

\section{THE EFFECT OF DIRECT PERITONEAL RESUSCITATION ON LEUKOCYTE ACTIVITY IN THE KIDNEY}

\section{Overview}

Background: Brain death is associated with significant inflammation and leukocyte activity within the peripheral organs including the kidneys. This inflammation is theorized to be the primary reason that multiple studies show reduced graft survival when transplanted organs are procured from brain dead donors. Therefore, reducing inflammatory mediators in brain dead donors and leukocyte infiltration into organs may improve transplant outcomes. Direct peritoneal resuscitation (DPR) has been shown to reduce systemic inflammation after brain death, but its effects on leukocytes within the kidney are not know.

Methods: Brain death was achieved in male Sprague-Dawley rats by inserting a 4F Fogarty catheter into the skull and inflating the balloon at $1 \mathrm{cc} / \mathrm{hr}$. Rats were resuscitated with normal saline to maintain a mean arterial pressure of $80 \mathrm{mmHg}$ (targeted intravenous fluid, TIVF) and DPR animals also received 30cc of intraperitoneal peritoneal dialysis solution. Rats were sacrificed at zero, two, four, and six hours after brain death and tissue samples taken. Protein levels were assessed using quantitative ELISA. Neutrophil and macrophage infiltration was assessed using flow cytometry. Results: Protein levels of adhesion molecules ICAM, VCAM, E-selectin, and P-selectin all increased after brain death, though in different time frames. Use of DPR caused a 
reduction in levels at each time point when compared to their respective TIVF groups. Flow cytometry revealed that the quantity of macrophages and neutrophils was equivalent in the TIVF and DPR groups at two and four hours after brain death, but at six hours after brain death the TIVF group had a large increase which was significantly decreased with DPR. Neutrophil myeloperoxidase activity was decreased with DPR, but macrophage activation was increased and two and four hours and equivalent at six hours. Conclusions: Use of DPR reduces systemic inflammation, leading to reduced adhesion molecule expression and decreasing leukocyte infiltration into the kidneys. Application of DPR to the resuscitation of brain dead donors has the potential to reduce the immunologic activity of transplanted kidneys and could lead to improved graft survival. 


\section{Background:}

The process of brain death is associated with significant inflammation $[15,16]$. This inflammation is frequently cited as the reason that transplanted organs from brain dead donors are associated with higher rates of graft failure $[16,60,109]$. The kidneys are not immune to this inflammation. Studies have shown increased cytokines and chemokines in human and rat kidneys after brain death at the time of procurement [59, 110]. This has lead a number of authors to suggest methods for targeting inflammatory mediators in an attempt to improve transplant outcomes [111]. Therapies such as dopamine [112], complement inhibitors [113], or vagal stimulation [114] have shown promise in animal models but have not gained widespread acceptance in the treatment of human organ donors.

Direct peritoneal resuscitation is an innovative method of resuscitation that improves blood flow to the visceral organs, but does not directly increase renal blood flow [21]. However, DPR has been shown to reduce systemic inflammatory cytokines after multiple types of shock [22, 26, 41, 115]. Using DPR in brain dead human donors has also been shown to increase the rate of organ procurement [27], suggesting that using DPR during resuscitation of brain dead donors exerts anti-inflammatory effects within the organs. However, no study has examined the effects of DPR specifically within the kidney. Kidneys are the most frequently transplanted organ [9], and leukocytes within transplanted organs have been associated with increased rates of rejection [79]. Therefore, the purpose of this study was to determine whether use of DPR would lead to a reduction in leukocyte infiltration into the kidney, as well as the mechanism through which it exerts its effects. 


\section{Methods:}

The methods were performed as explained in Chapter 2: Materials and Methods. In brief, our murine model of brain death was used and rats were sacrificed immediately after brain death (time zero) as well as two, four, and six hours after brain death. Those scheduled for DPR received a single intraperitoneal injection of $30 \mathrm{~mL}$ of Delflex solution. Prior to brain death rats were resuscitated with enough normal saline to maintain a MAP of $80 \mathrm{mmHg}$ and vitals were measured continuously. After sacrifice, kidney tissue was flash frozen in liquid nitrogen and ultimately used for protein analysis with ELISA, or digested in collagenase for creation of a single cell suspension for flow cytometry.

\section{Results:}

Results of protein levels of ICAM and VCAM are shown in figures 23 and 24 while protein levels of E-selectin and P-selectin are shown in figures 25 and 26. Both ICAM and VCAM peak at two hours but are increased above sham at two, four, and six hours. Use of DPR reduced ICAM and VCAM to sham levels at all time points when compared to the respective TIVF groups. E-selectin was increased at four and six hours after brain death and reduced at both time points with DPR. P-selectin was increased at zero, two, four, and six hours compared to sham and reduced with DPR at two, four, and six hours. 


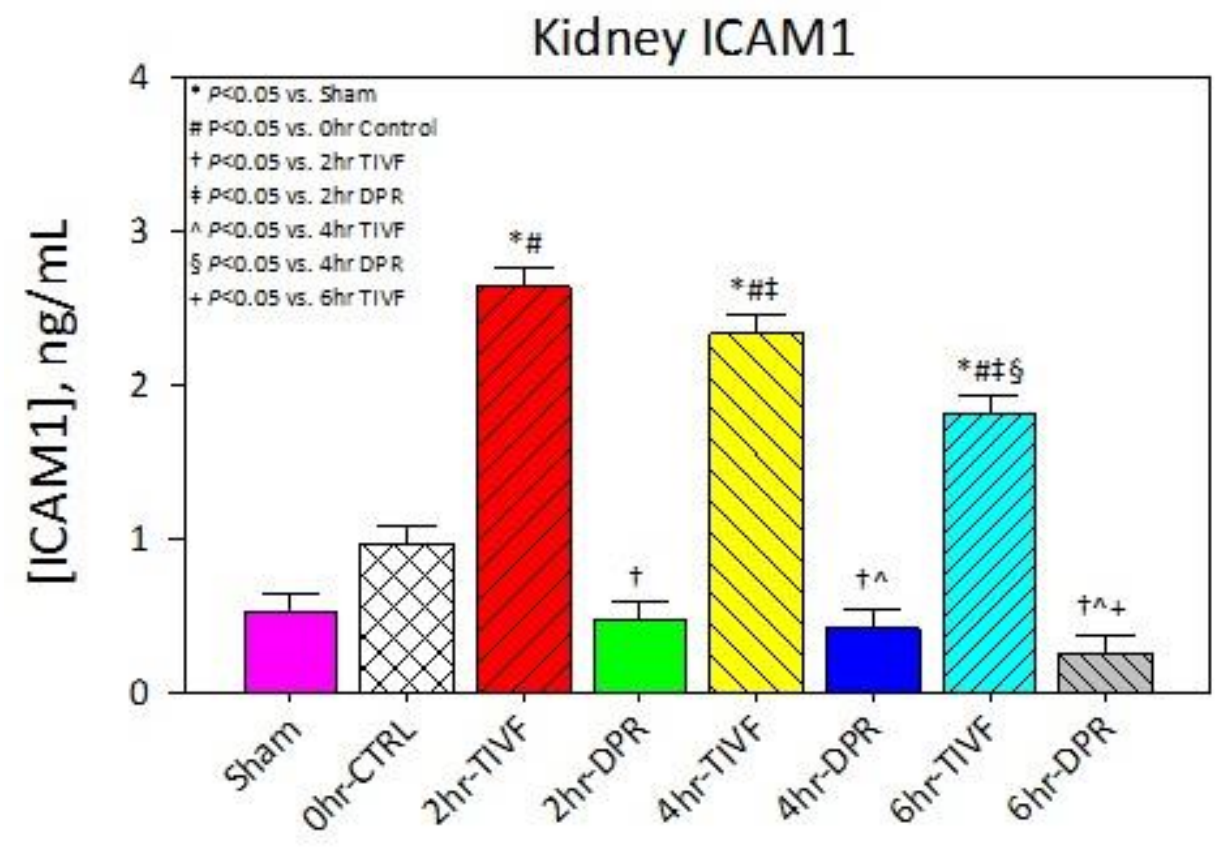

Figure 23: Levels of ICAM protein in kidney tissue in sham rats and at 0, 2, 4, and 6 hours after brain death with and without DPR. 


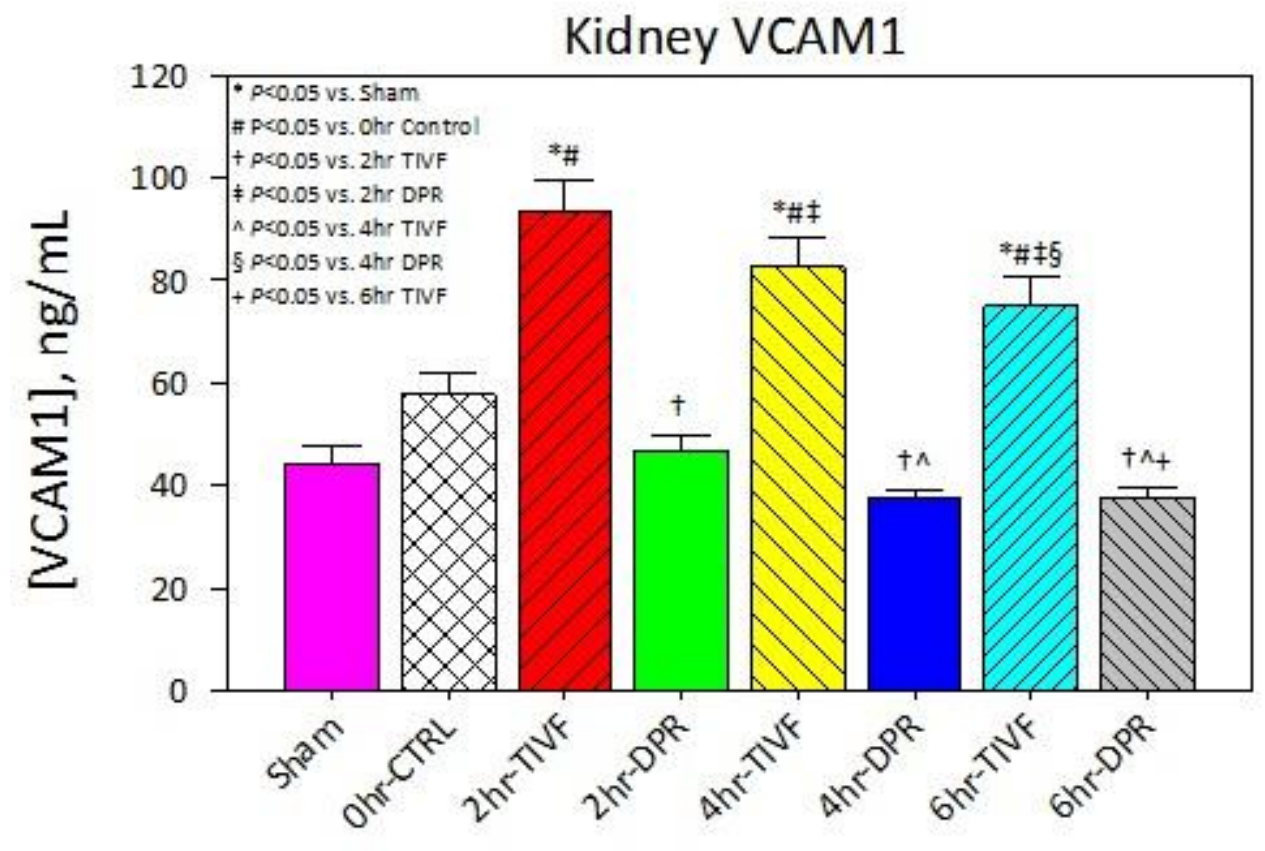

Figure 24: Levels of VCAM protein in kidney tissue in sham rats and at 0, 2, 4, and 6 hours after brain death with and without DPR. 


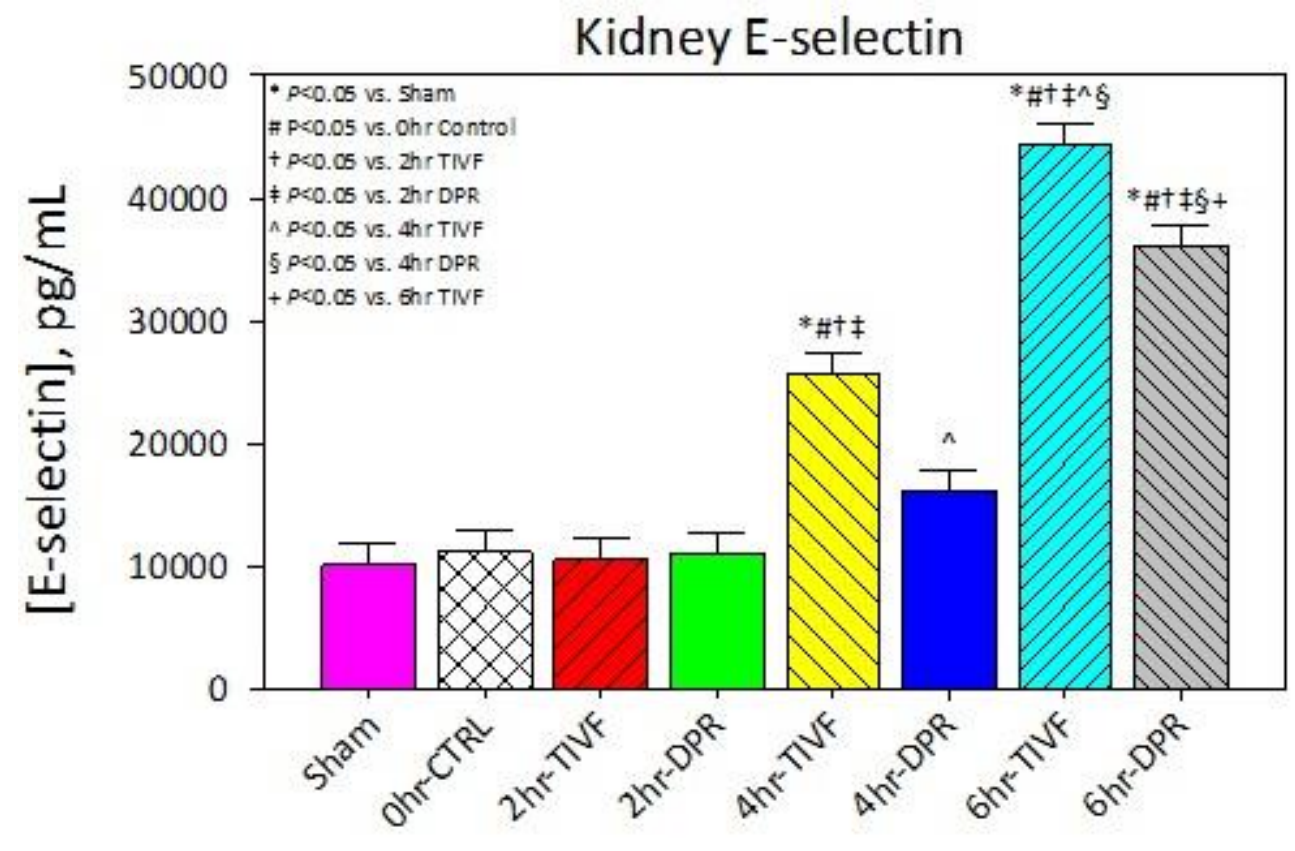

Figure 25: Levels of E-selectin protein in kidney tissue in sham rats and at 0,2, 4, and 6 hours after brain death with and without DPR. 


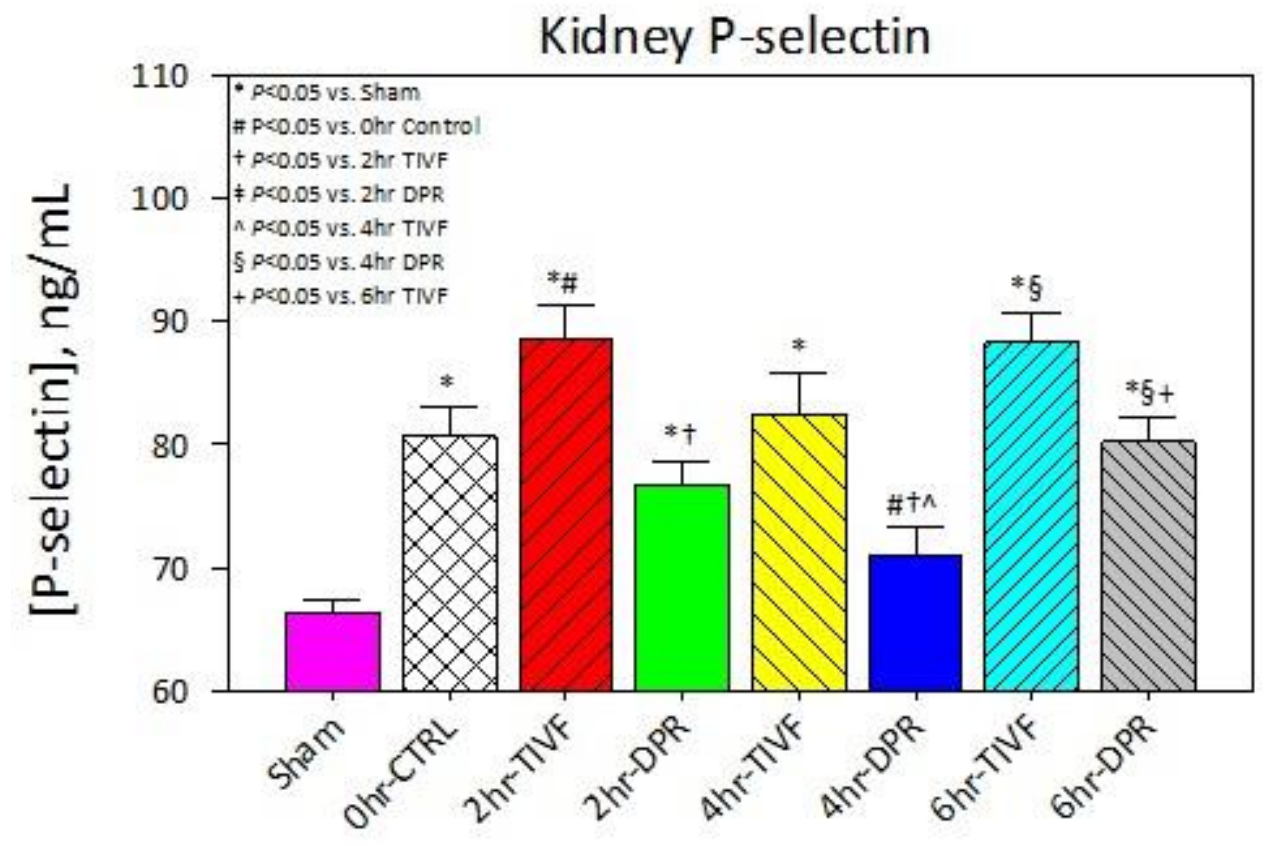

Figure 26: Levels of P-selectin protein in kidney tissue in sham rats and at 0, 2, 4, and 6 hours after brain death with and without DPR. 
The quantification of neutrophils by flow cytometry is shown in Figure 27. The DPR and TIVF groups were equivalent to each other at two and four hours, but at six hours the use of DPR significantly reduced the increase in neutrophils seen in the TIVF group. The MPO assay shows that neutrophil activation was increased by the process of brain death itself, increasing above sham levels in the time zero animals (Figure 28). Neutrophil activity was further increased at four and six hours above the time zero levels, and decreased at both those time points with the addition of DPR.

Macrophage infiltration is shown in Figure 29. Like the neutrophils, the two experimental groups were equivalent at two and four hours. However, the increase in macrophages in the TIVF group is significantly reduced in the DPR group. Macrophage M1 activation was unchanged by DPR (Figure 30). Conversely, M2 activation was equivalent between the TIVF and DPR groups at two and four hours, but significantly decreased by DPR at six hours. 


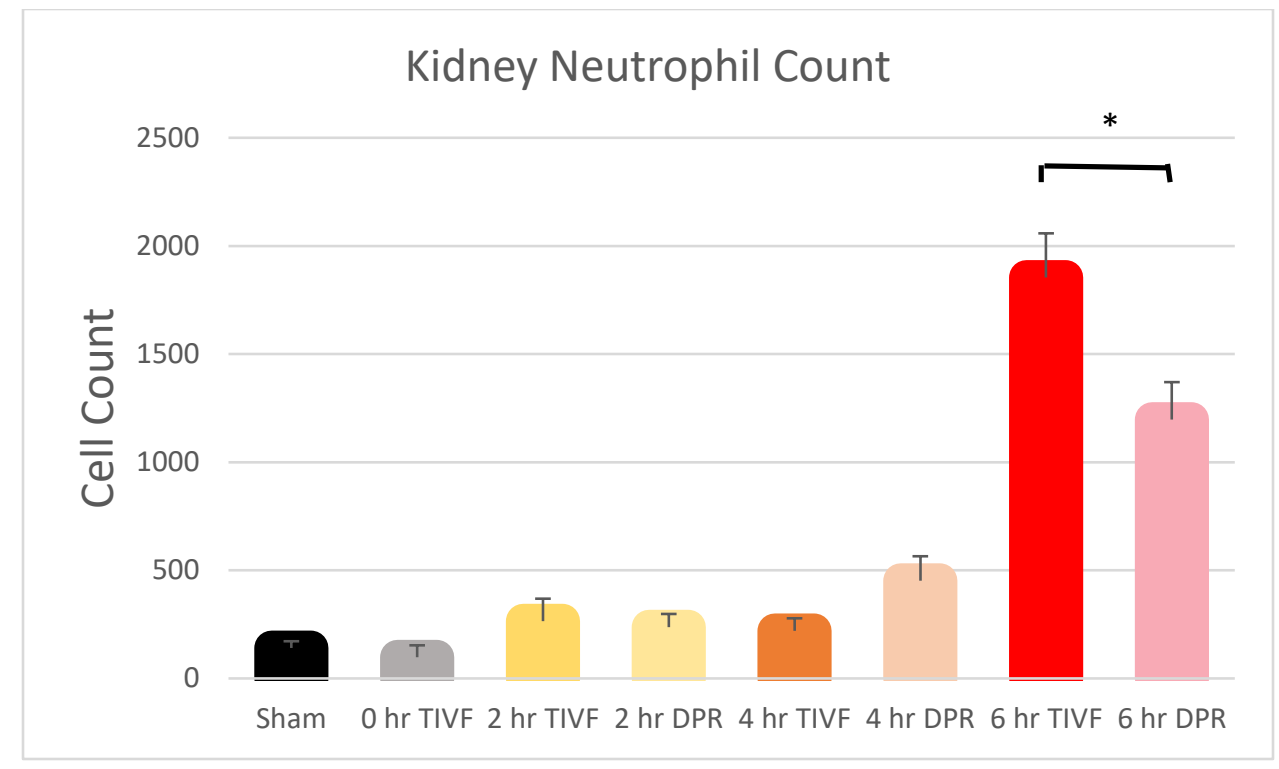

Figure 27: Flow cytometry results of neutrophil infiltration into the kidney at 0,2, 4, and 6 hours after brain death with and without DPR. *p $<0.05$ vs DPR group at same time point. 


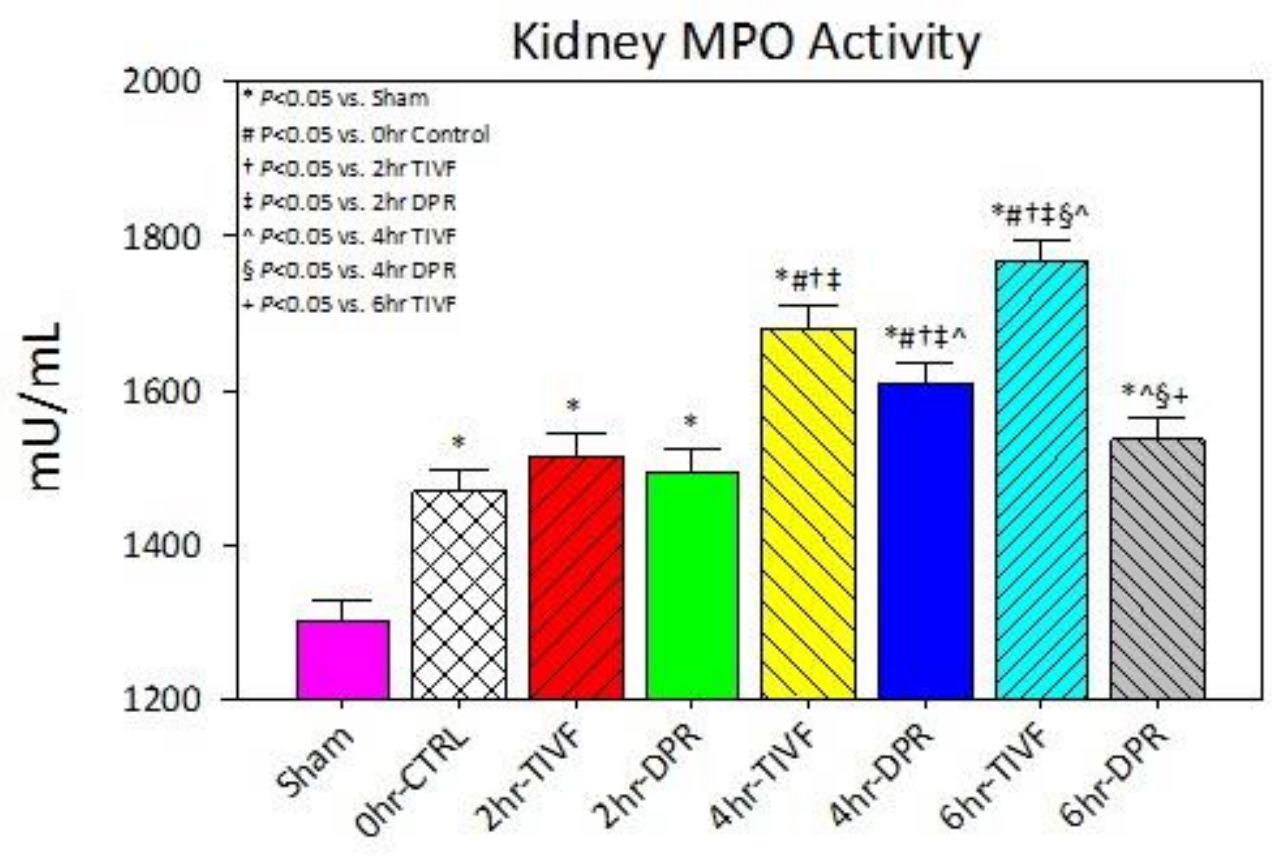

Figure 28: Myeloperoxidase activity assay in the kidney in sham rats and at $0,2,4$, and 6 hours after brain death with and without DPR. 


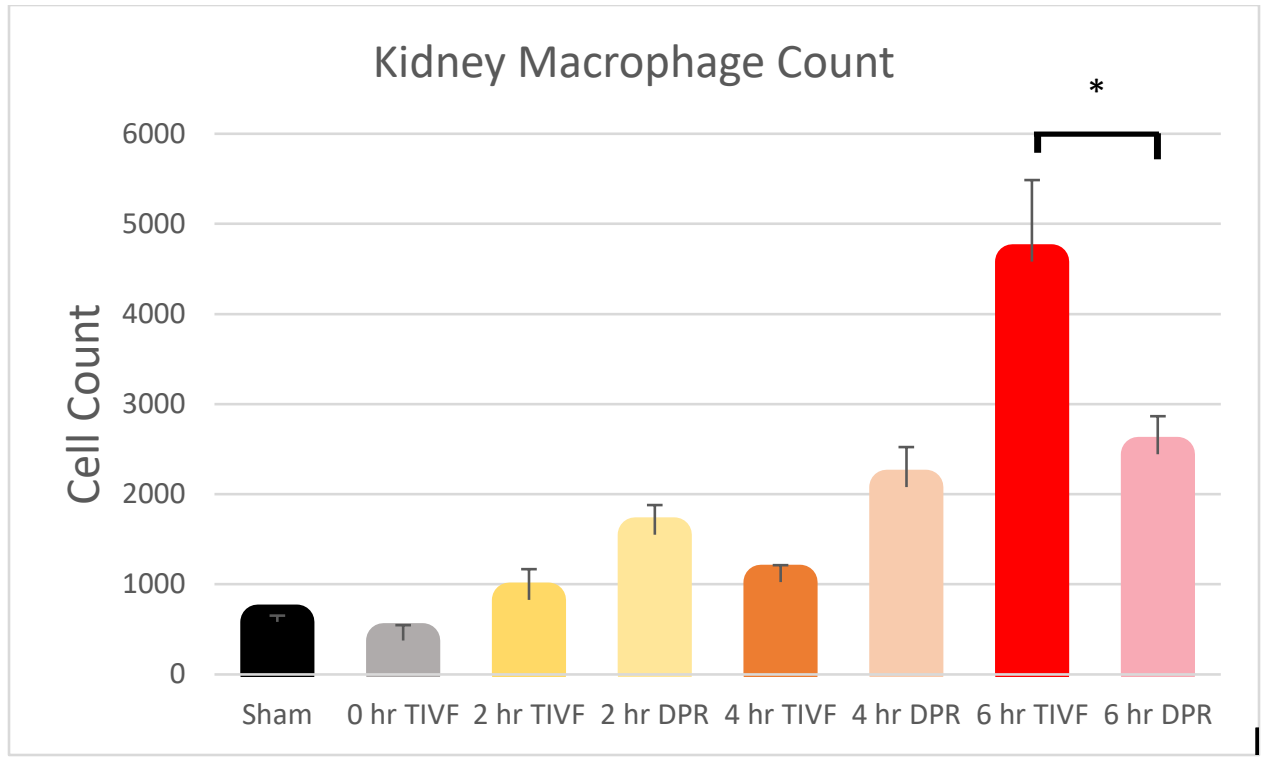

Figure 29: Flow cytometry results of macrophage infiltration into the kidney at 0, 2, 4, and 6 hours after brain death with and without DPR. *p $<0.05$ vs DPR group at same time point. 

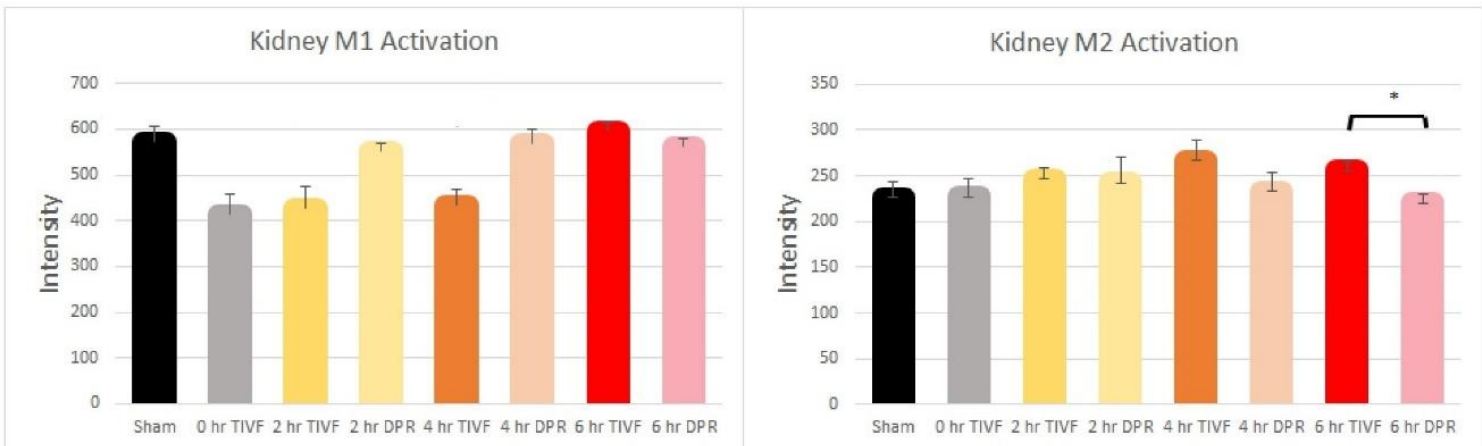

Figure 30: Flow cytometry results showing macrophage activity in the kidney at 0, 2, 4, and 6 hours after brain death with and without DPR. *p $<0.05$ vs DPR group at same time point. 
Protein levels of cytokine within the kidney tissue are displayed in Table 5. Levels of pro-inflammatory TNF- $\alpha$ and IL-6 were increased at four and six hours, but TNF- $\alpha$ was only significantly decreased by DPR at four hours while IL- 6 was decreased at both time points. Levels of IL-1 $\alpha$, IL-12, and IL-13 were not significantly changed at any time point, while IL- $1 \beta$ increased at two hours and then dramatically at six hours, and was decreased at both these time points with DPR. Interleukin 2 was increased starting at two hours and unchanged by DPR, while IL-18 and IFN- $\gamma$ were increased above sham at two, four, and six hours but only reduced by DPR at some of these times. Chemokine CCL-2 was statistically increased at two hours, and then again more significantly at six hours, while CCL-3 was increased starting at two hours and dramatically reduced by DPR. 


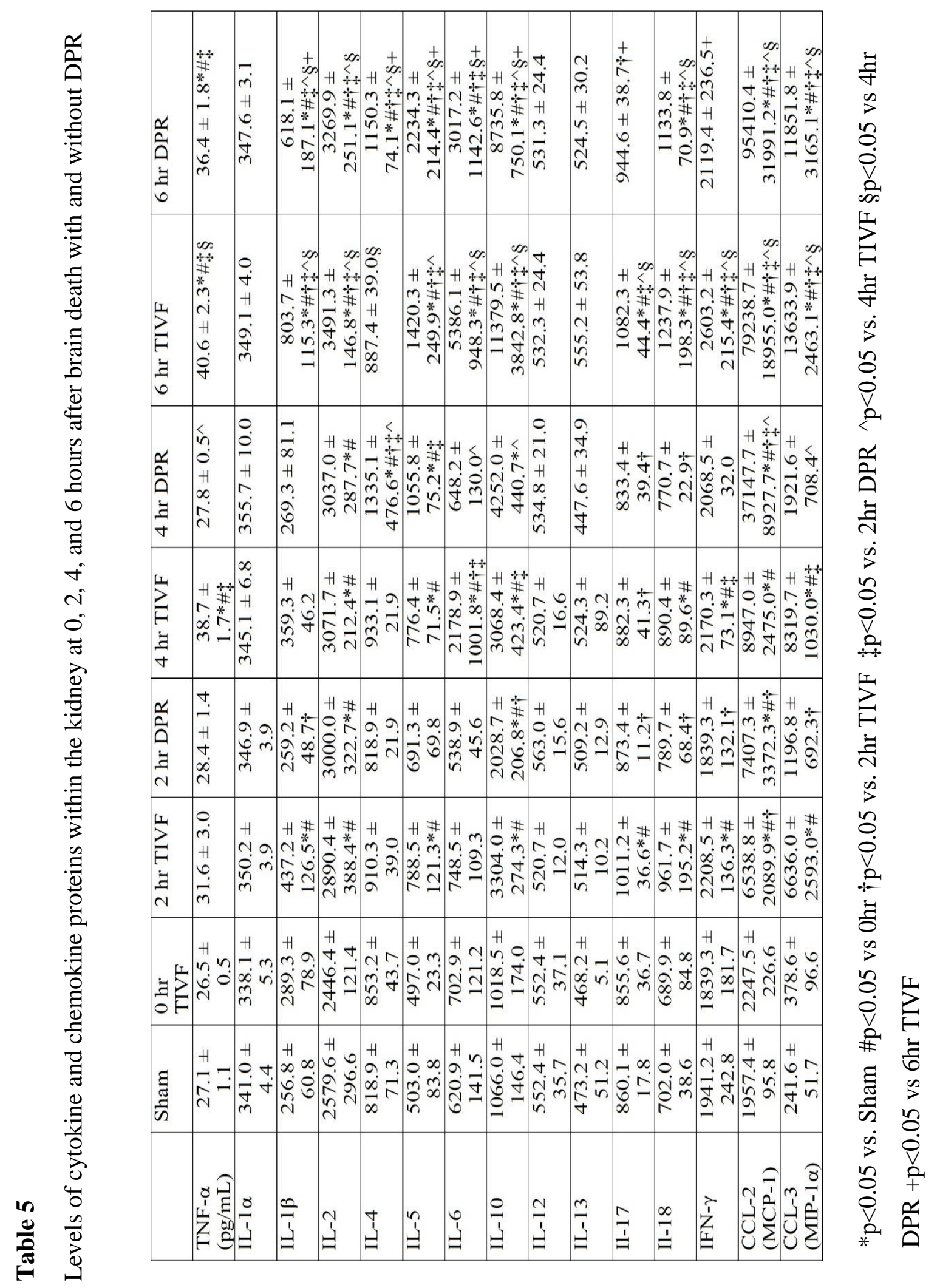




\section{Discussion:}

Kidneys are the most frequently transplanted organ in the United States [9]. However, in 2009 there were over 871,000 people in the US requiring dialysis due to renal failure, many of whom would benefit from transplantation [116]. Unfortunately, those who receive a transplant do not always remain off dialysis, as the number of grafts still functioning 10 years after transplant is on average only $34-48 \%$ [117]. The most common reason for graft failure is chronic graft rejection, followed by glomerulonephritis and infection [118]. Kidneys procured from brain dead donors are associated with worse long-term outcomes than those from other donors, worsening this problem [60].

This study helps delineate the inflammatory process within the kidney during brain death. Much like within the lung, a number of inflammatory cytokines increase within the kidney tissue within two hours of brain death, including IL-1 $\beta$, IL-2, IL-17, IL-18, and IFN- $\gamma$. However, IL-6 and TNF- $\alpha$ take four hours and IL-1 $\alpha$ does not change at all. It appears that overall, the kidney is less responsive to the process of brain death than the lungs, at least over the period studied. This could be in part because of the significant blood flow to the kidneys, especially in brain death as there is a loss of the neural and hormonal signals that typically cause renal vasoconstriction.

These inflammatory cytokines are one of the triggers for leukocyte adhesion molecules. Previous authors have measured levels of adhesion molecules within the kidney after brain death and found varied results. Skrabal et al. found no difference in ICAM levels at six hours in a pig model of brain death [119], while van der Hoeven and colleagues found that ICAM and VCAM were increased in rats at six hours but not at one hour after brain death based on immunohistochemistry staining [120]. In humans, brain 
death leads to an increase in E-selectin expression at the time of organ procurement [59], while Schuurs and associates saw increased E-selectin as early as 30 minutes after brain death in rats [73]. In contrast, this study not only looks as multiple time points, unlike most of these studies, but also measures actual protein levels instead of mRNA to quantify these adhesion molecules. These findings demonstrate that ICAM and VCAM are increased as soon as two hours after brain death, and P-selectin is increased immediately after brain death, while E-selectin takes four hours to increase.

Use of DPR downregulates the expression of all of these molecules, likely by downregulating their primary stimuli. There are many triggers for increased expression of these adhesion molecules, including most commonly endothelial injury and release of inflammatory cytokines such as IL-1 and TNF- $\alpha$ [99, 121, 122]. We have previously demonstrated that use of DPR downregulates these mediators in rat serum [41], by improving intestinal blood flow [21] and preventing increased intestinal permeability as shown in chapter 4 . These data also demonstrate reductions in TNF- $\alpha$ and IL-1 $\beta$ within the kidney tissue. Thus, by preventing this stimulation, DPR prevents the upregulation of leukocyte adherence proteins.

The level of adherence proteins correlates directly with leukocyte infiltration. The timing for this infiltration has been varied in different experiments looking at brain death. For example, infiltration of macrophages and neutrophils into the kidney was observed at six hours by Takada et al. [15] while Schuurs and colleagues found an increase in cortical neutrophils that began at 30 minutes and peaked at two hours after brain death, as assessed by immunohistochemistry [73]. These data show a definitive change by six hours after brain death in both macrophage and neutrophils. Prior to six 
hours, the TIVF group is equivalent to sham, and DPR causes no change. However, while the significant infiltration occurs at six hours in the TIVF rats, use of DPR is able to significantly reduce this increase. This suggests that the use of DPR leads to a significant reduction, or at least a delay, in the infiltration of leukocytes into the kidney. While causation has not been definitively proven, it is likely that this is due to the reduction in adhesion molecules which are necessary for sequestration. Procurement of organs prior to this time in rats, or in the equivalent time of approximately 18-24 hours in humans, may lead to transplantation of leukocyte-reduced organs and the potential for reduced immunogenicity. However, more study is needed to verify these hypotheses.

The flow cytometry demonstrates a dramatic increase in macrophage and neutrophil infiltration into the rat kidney between four and six hours after brain death. This is different than most findings that have examined leukocyte infiltration after other types of shock. Awad et al. found that neutrophils had increased in the mouse kidney $210 \%$ above baseline only two hours after ischemia and reperfusion [108]. Ysebaert and colleagues found increased MPO activity in rats within one hour of reperfusion, but noted only rare neutrophils on H\&E staining [123]. They also found a gradual increase in macrophages over the first 12 hours. Other authors found infiltration to occur prior to three and five hours after hemorrhage [84, 87]. Conversely, Langenberg et al. found no histologic changes in the kidneys of sheep 48 hours after infusion of IV Escherichia coli simulating septic shock [124]. The variability of these different experiments, including use of different species, different models, and the intrinsic variability with any in vivo model, makes the determination of a specific time for leukocyte infiltration difficult. However, the majority suggest that infiltration occurs earlier after hemorrhagic shock and 
ischemia/reperfusion than the current findings show brain death. This implies that the inflammatory process associated with brain death is not merely due to ischemia and reperfusion during the process of herniation or resuscitation, but is fundamentally different. This difference could be due to the other changes that occur with brain death, such as endocrine changes, hormone levels, changes in catecholamine release, or some unknown factor released during the process. Further studies are needed to determine which, if any, of these is a factor in brain death.

While DPR causes a reduction in the quantity of macrophages moving into the tissues, it does not reduce the degree of macrophage activation. Macrophage activation is a complex process that is divided into $\mathrm{M} 1$, or classical activation, and M2 activation, which is typically more anti-inflammatory. Classical activation is triggered by INF- $\gamma$ followed by interaction with specific molecules such as toll-like receptors [48]. In this study we found that DPR caused a temporary increase in M1 activation at two and four hours, and the groups became equivalent at six hours. In a previous study we found that use of DPR causes an increase in IFN- $\gamma$ at two hours [41], which may explain this finding. On the other hand, use of DPR did reduce myeloperoxidase activity, which reflects neutrophil activity. This may represent the reduction in neutrophil quantity, or may reflect a reduction in neutrophil activity, or both. Neutrophils are stimulated by a number of factors, including IL-8, platelet activating factor, leukotrienes, and complement proteins [125], and the majority of these have not yet been tested but may be affected by DPR. Further study will be needed to determine the mechanism that alters neutrophil function. 
A direct comparison between the kidney and lung results is difficult, as different amounts of tissue were used for the different experiments. However, some interesting trends are suggested. As mentioned earlier, it appears based on the behavior of individual cytokines that the kidney has a more blunted initial inflammatory response compared to the lungs. However, despite the lower and slower initial stimulus, the kidney appears to have an even more dramatic response in terms of leukocyte infiltration. For example, macrophages within the lung increase 2.2 times in six hours but in the kidney they increase over seven-fold. Compared to the lung, the adhesion molecules within the kidney generally increased earlier, so it is possible that the inflammatory process within the kidney occurs faster and thus is more advanced by six hours, and that the time points chosen did not adequately capture this initial inflammatory response. Another possibility is that this may also be a consequence of differences between the two organs themselves: the lung tissue is typically full of resident macrophages, and the lungs are constantly assaulted by stimuli through their connection to the outside world, which may make them more accustomed to a constant low level of inflammation and require a larger stimulus. Alternatively, the presence of macrophages within the kidney is generally associated with pathology, and thus the kidney tissue may only require a smaller stimulus to produce a big leukocyte response.

Multiple investigators have attempted to reduce the inflammatory activity in the kidneys associated with brain death by targeting cytokines, leukocytes, or other stimuli. However, no method has gained widespread use in the medical community. This study both delineates the timeline of leukocyte infiltration into the kidney after brain death and suggest a new way to counteract it. Using DPR is a novel way of improving post-brain 
death inflammation by preventing the intestinal ischemia that triggers the release of some of these inflammatory mediators and prevents the stimulation of adhesion molecules and subsequent leukocyte infiltration.

\section{Conclusions}

Use of DPR causes a significant reduction in expression of ICAM, VCAM, E-selectin, and P-selectin, likely by reducing their primary stimuli. This in turn likely causes the significant reduction in macrophage and neutrophil infiltration into the kidney. However, DPR does not appear to alter the course of macrophage activation. While these findings represent only preliminary data, this suggests that use of DPR could lead to the ability to procure leukocyte-reduced organs for transplantation. 


\section{CHAPTER 7}

\section{CONCLUSIONS}

The complex physiologic processes associated with the progression to brain death lead to significant systemic inflammation. We have provided a clear timeline and suggested a mechanism for how this occurs (Figure 31). Brain death is associated with profound hypotension that leads to intestinal ischemia. This in turn leads to increased intestinal permeability, allowing for the release of more inflammatory cytokines and DAMPs such as HMGB-1, and HSP70. This systemic increase begins within two hours of brain death. These act as signals to stimulate the expression of leukocyte adhesion molecules within the lungs and kidneys between two and four hours after brain death. Finally, these changes in leukocyte adhesion molecules leads to an increase in leukocyte infiltration into the tissues by six hours. Direct peritoneal resuscitation prevents the intestinal ischemia that initiates this progression, thus reducing the macrophages and neutrophils in lungs and kidneys after brain death. 

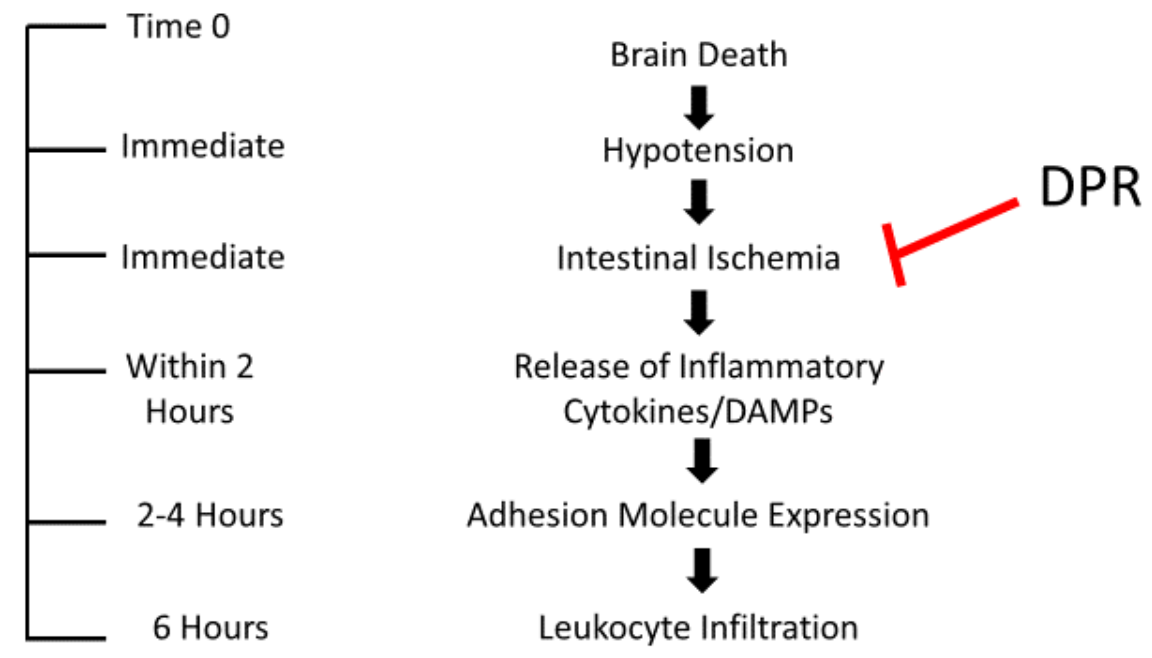

Figure 31. Schematic diagram showing the proposed sequence of events and relevant time points of inflammation and leukocyte infiltration after brain death. DPR alters this course by reversing the intestinal ischemia after brain death. 
In addition, we showed that DPR does not have a prolonged effect on the activation of macrophages. Since DPR is applied after brain death has occurred, it appears that this is too late to prevent this activation. Other factors we examined, such as some of the leukocyte adhesion molecules, showed a significant difference between those measured in sham animals when compared to those measured immediately after brain death, suggesting that the process of brain death itself induces some significant and immediate changes within the organs. This suggests that it may not be possible to fully prevent the rise in systemic inflammation that occurs after brain death, or that to do so may require intervention before brain death occurs. While DPR has clear limitations to its application and effects, it shows considerable potential for its use in reducing inflammation in brain death.

\section{Limitations}

These studies have multiple limitations. First, the groups are relatively small for an in vivo model which is intrinsically variable. This may be why some of the observed differences failed to reach statistical significance. Next, these observations are limited to the first six hours after brain death, largely because with time the rats used become increasingly unstable, making longer observation periods difficult. It is possible that after this time point subsequent measurements would show that the groups become equivalent, or that DPR would have a different effect. In addition, the lack of further time points means that we can only state that using DPR reduces leukocyte at the times measured, and it is possible that using DPR only delays this infiltration, which cannot be seen without a longer observation period. While our data find a strong association between intestinal permeability and systemic inflammation, this causation is not definitively 
proven. The effects of DPR on both are certainly suggestive of a relationship, but further study would be needed to clearly demonstrate a cause and effect. Also, while we have demonstrated that use of DPR in brain dead donors offers an opportunity to procure leukocyte-reduced organs, it has not been proven that this offers any long-term benefit to the transplant recipient. Finally, as this is an animal study, similar studies in humans would be necessary demonstrate whether these findings are also valid in human subjects.

\section{Future Directions}

There are several next steps for this work. One is to definitely demonstrate the connection between brain death and intestinal permeability which can be done in an in vivo model using a marker such as Evan's blue dye or labeled albumin infused prior to brain death, and then measuring it in the intestine before and after brain death. In order to definitively show the connection between inflammatory cytokines, adhesion molecules, and leukocyte infiltration, antibodies to inflammatory cytokines or the leukocyte adhesion molecules could be used to block and individual step to determine whether a subsequent step is affected as expected. Finally, an animal model of lung and kidney transplantation could be used to determine whether the leukocyte reduction prior to procurement is maintained after transplantation. 


\section{REFERENCES}

1. Get the Stats on Traumatic Brain Injury in the United States, C.f.D.C.a. Prevention, Editor.

2. Ponsford, J.L., et al., Costs of Care after Traumatic Brain Injury. Journal of Neurotrauma, 2013. 30: p. 1498-1505.

3. Dessy, A., et al., A review of modifying factors affecting usage of diagnostic rating scales in concussion management. Clinical Neurology and Neurosurgery, 2014. 122: p. 59-63.

4. B, Y., B. W, and L. A, Brain death and the persistent vegetative state: similarities and contrasts. Canadian Journal of Neurological Sciences, 1989. 16(4): p. 388-93.

5. Tran, L.V., Understanding the pathophysiology of traumatic brain injury and the mechanisms of action of neuroprotective interventions. J Trauma Nurs, 2014. 21(1): p. 30-5.

6. Smith, M., Physiologic changes during brain stem death--lessons for management of the organ donor. J Heart Lung Transplant, 2004. 23(9 Suppl): p. S217-22.

7. Kutsogiannis, D.J., et al., Medical management to optimize donor organ potential: review of the literature. Can J Anaesth, 2006. 53(8): p. 820-30.

8. Salim, A., et al., The effect of a protocol of aggressive donor management: Implications for the national organ donor shortage. J Trauma, 2006. 61(2): p. 429-33; discussion 4335.

9. Wong, C.J. and G. Pagalilauan, Primary Care of the Solid Organ Transplant Recipient. Med Clin North Am, 2015. 99(5): p. 1075-103.

10. Da Silva, I.R. and J.A. Frontera, Worldwide barriers to organ donation. JAMA Neurol, 2015. 72(1): p. 112-8.

11. Hassoun, H.T., et al., Post-injury multiple organ failure: the role of the gut. Shock, 2001. 15(1): p. 1-10.

12. Moore, F.A., The role of the gastrointestinal tract in postinjury multiple organ failure. Am J Surg, 1999. 178(6): p. 449-53.

13. Carrico, C.J., et al., Multiple-organ-failure syndrome. Arch Surg, 1986. 121(2): p. 196208.

14. Swank, G.M. and E.A. Deitch, Role of the gut in multiple organ failure: bacterial translocation and permeability changes. World J Surg, 1996. 20(4): p. 411-7.

15. Takada, M., et al., Effects of explosive brain death on cytokine activation of peripheral organs in the rat. Transplantation, 1998. 65(12): p. 1533-42.

16. Barklin, A., Systemic inflammation in the brain-dead organ donor. Acta Anaesthesiol Scand, 2009. 53(4): p. 425-35.

17. McKeating, E.G., et al., Transcranial cytokine gradients in patients requiring intensive care after acute brain injury. Br J Anaesth, 1997. 78(5): p. 520-3.

18. Watts, R.P., O. Thom, and J.F. Fraser, Inflammatory signalling associated with brain dead organ donation: from brain injury to brain stem death and posttransplant ischaemia reperfusion injury. J Transplant, 2013. 2013: p. 521369.

19. Miller, F.N., et al., Hyperosmolality, acetate, and lactate: dilatory factors during 
peritoneal dialysis. Kidney Int, 1981. 20(3): p. 397-402.

20. Zakaria el, R., et al., Generalized dilation of the visceral microvasculature by peritoneal dialysis solutions. Perit Dial Int, 2002. 22(5): p. 593-601.

21. Zakaria el, R., et al., A novel method of peritoneal resuscitation improves organ perfusion after hemorrhagic shock. Am J Surg, 2003. 186(5): p. 443-8.

22. Garrison, R.N., et al., Direct peritoneal resuscitation as adjunct to conventional resuscitation from hemorrhagic shock: a better outcome. Surgery, 2004. 136(4): p. 9008.

23. Garrison, R.N. and R. Zakaria el, Peritoneal resuscitation. Am J Surg, 2005. 190(2): p. 1815.

24. Zakaria el, R., et al., Intraperitoneal resuscitation improves intestinal blood flow following hemorrhagic shock. Ann Surg, 2003. 237(5): p. 704-11; discussion 711-3.

25. Smith, J.W., et al., Direct peritoneal resuscitation accelerates primary abdominal wall closure after damage control surgery. J Am Coll Surg, 2010. 210(5): p. 658-64, 664-7.

26. Smith, J.W., et al., Adjunctive treatment of abdominal catastrophes and sepsis with direct peritoneal resuscitation: indications for use in acute care surgery. J Trauma Acute Care Surg, 2014. 77(3): p. 393-8; discussion 398-9.

27. Smith, J.W., et al., Addition of direct peritoneal lavage to human cadaver organ donor resuscitation improves organ procurement. J Am Coll Surg, 2015. 220(4): p. 539-47.

28. Benhamou, Y., et al., Toll-like receptors 4 contribute to endothelial injury and inflammation in hemorrhagic shock in mice. Crit Care Med, 2009. 37(5): p. 1724-8.

29. Seal, J.B. and B.L. Gewertz, Vascular dysfunction in ischemia-reperfusion injury. Ann Vasc Surg, 2005. 19(4): p. 572-84.

30. Carden, D.L. and D.N. Granger, Pathophysiology of ischaemia-reperfusion injury. J Pathol, 2000. 190(3): p. 255-66.

31. Weaver, J.L., et al. DPR Reduces Visceral Ischemia and Inflammatory Cytokines Following Hemorrhagic Shock. in Academic Surgical Congress. 2014. Las Vegas, NV.

32. Weaver, J.L. and J.W. Smith, Direct Peritoneal Resuscitation: A review. Int J Surg, 2015.

33. Anders, H.J. and L. Schaefer, Beyond tissue injury-damage-associated molecular patterns, toll-like receptors, and inflammasomes also drive regeneration and fibrosis. J Am Soc Nephrol, 2014. 25(7): p. 1387-400.

34. Chan, J.K., et al., Alarmins: awaiting a clinical response. J Clin Invest, 2012. 122(8): p. 2711-9.

35. Peltz, E.D., et al., HMGB1 is markedly elevated within 6 hours of mechanical trauma in humans. Shock, 2009. 32(1): p. 17-22.

36. Tsung, A., S. Tohme, and T.R. Billiar, High-mobility group box-1 in sterile inflammation. J Intern Med, 2014. 276(5): p. 425-43.

37. Yu, Y., D. Tang, and R. Kang, Oxidative stress-mediated HMGB1 biology. Front Physiol, 2015. 6: p. 93.

38. Bianchi, M.E., DAMPs, PAMPs and alarmins: all we need to know about danger. J Leukoc Biol, 2007. 81(1): p. 1-5.

39. Laurent, T.C., U.B. Laurent, and J.R. Fraser, Serum hyaluronan as a disease marker. Ann Med, 1996. 28(3): p. 241-53.

40. Berg, S., et al., Elevated levels of plasma hyaluronan in septicaemia. Scand J Clin Lab Invest, 1988. 48(8): p. 727-32. 
41. Smith, J.W., et al., Direct peritoneal resuscitation improves inflammation, liver blood flow, and pulmonary edema in a rat model of acute brain death. J Am Coll Surg, 2014. 219(1): p. 79-87.

42. Tecchio, C., A. Micheletti, and M.A. Cassatella, Neutrophil-derived cytokines: facts beyond expression. Front Immunol, 2014. 5: p. 508.

43. Cassatella, M.A., The production of cytokines by polymorphonuclear neutrophils. Immunol Today, 1995. 16(1): p. 21-6.

44. Labonte, A.C., A.C. Tosello-Trampont, and Y.S. Hahn, The role of macrophage polarization in infectious and inflammatory diseases. Mol Cells, 2014. 37(4): p. 275-85.

45. Jani, P.K., et al., MASP-1 induces a unique cytokine pattern in endothelial cells: a novel link between complement system and neutrophil granulocytes. PLoS One, 2014. 9(1): p. e87104.

46. Silva, M.T., Neutrophils and macrophages work in concert as inducers and effectors of adaptive immunity against extracellular and intracellular microbial pathogens. J Leukoc Biol, 2010. 87(5): p. 805-13.

47. Silva, M.T., When two is better than one: macrophages and neutrophils work in concert in innate immunity as complementary and cooperative partners of a myeloid phagocyte system. J Leukoc Biol, 2010. 87(1): p. 93-106.

48. Wyburn, K.R., et al., The role of macrophages in allograft rejection. Transplantation, 2005. 80(12): p. 1641-7.

49. Magil, A.B., Monocytes/macrophages in renal allograft rejection. Transplant Rev (Orlando), 2009. 23(4): p. 199-208.

50. Spahn, J.H., W. Li, and D. Kreisel, Innate immune cells in transplantation. Curr Opin Organ Transplant, 2014. 19(1): p. 14-9.

51. El-Sawy, T., et al., Inhibition of polymorphonuclear leukocyte-mediated graft damage synergizes with short-term costimulatory blockade to prevent cardiac allograft rejection. Circulation, 2005. 112(3): p. 320-31.

52. Skrajnar, S., M. Anzur Lasnik, and A. Bedina Zavec, A flow cytometric method for determination of the blood neutrophil fraction in rats. J Am Assoc Lab Anim Sci, 2009. 48(2): p. 152-6.

53. Ritter, L., et al., Exaggerated neutrophil-mediated reperfusion injury after ischemic stroke in a rodent model of type 2 diabetes. Microcirculation, 2011. 18(7): p. 552-61.

54. Badylak, S.F., et al., Macrophage phenotype as a determinant of biologic scaffold remodeling. Tissue Eng Part A, 2008. 14(11): p. 1835-42.

55. Graversen, J.H., et al., Targeting the hemoglobin scavenger receptor CD163 in macrophages highly increases the anti-inflammatory potency of dexamethasone. Mol Ther, 2012. 20(8): p. 1550-8.

56. Wilhelm, M.J., et al., Activation of the heart by donor brain death accelerates acute rejection after transplantation. Circulation, 2000. 102(19): p. 2426-33.

57. Avlonitis, V.S., et al., The hemodynamic mechanisms of lung injury and systemic inflammatory response following brain death in the transplant donor. Am J Transplant, 2005. 5(4 Pt 1): p. 684-93.

58. Weiss, S., et al., Brain death activates donor organs and is associated with a worse $I / R$ injury after liver transplantation. Am J Transplant, 2007. 7(6): p. 1584-93.

59. Nijboer, W.N., et al., Effects of brain death on stress and inflammatory response in the human donor kidney. Transplant Proc, 2005. 37(1): p. 367-9. 
60. Floerchinger, B., R. Oberhuber, and S.G. Tullius, Effects of brain death on organ quality and transplant outcome. Transplant Rev (Orlando), 2012. 26(2): p. 54-9.

61. Rupani, B., et al., Relationship between disruption of the unstirred mucus layer and intestinal restitution in loss of gut barrier function after trauma hemorrhagic shock. Surgery, 2007. 141(4): p. 481-9.

62. Clark, J.A. and C.M. Coopersmith, Intestinal crosstalk: a new paradigm for understanding the gut as the "motor" of critical illness. Shock, 2007. 28(4): p. 384-93.

63. Erces, D., et al., Complement C5a inhibition improves late hemodynamic and inflammatory changes in a rat model of nonocclusive mesenteric ischemia. Surgery, 2016. 159(3): p. 960-71.

64. Vincenti, M., et al., Induction of intestinal ischemia reperfusion injury by portal vein outflow occlusion in rats. J Gastroenterol, 2010. 45(11): p. 1103-10.

65. Cheng, S., et al., Serologic Intestinal-Fatty Acid Binding Protein in Necrotizing Enterocolitis Diagnosis: A Meta-Analysis. Biomed Res Int, 2015. 2015: p. 156704.

66. Pelsers, M.M., W.T. Hermens, and J.F. Glatz, Fatty acid-binding proteins as plasma markers of tissue injury. Clin Chim Acta, 2005. 352(1-2): p. 15-35.

67. Leber, B., et al., Effect of oxidative stress and endotoxin on human serum albumin in brain-dead organ donors. Transl Res, 2012. 159(6): p. 487-96.

68. Simas, R., et al., Paradoxical effects of brain death and associated trauma on rat mesenteric microcirculation: an intravital microscopic study. Clinics (Sao Paulo), 2012. 67(1): p. 69-75.

69. Koudstaal, L.G., et al., Increased intestinal permeability in deceased brain dead rats. Transplantation, 2009. 88(3): p. 444-6.

70. Wilhelm, M.J., et al., Activation of proinflammatory mediators in heart transplants from brain-dead donors: evidence from a model of chronic rat cardiac allograft rejection. Transplant Proc, 2002. 34(6): p. 2359-60.

71. Danobeitia, J.S., et al., Early activation of the inflammatory response in the liver of braindead non-human primates. J Surg Res, 2012. 176(2): p. 639-48.

72. Wauters, S., et al., Evaluating lung injury at increasing time intervals in a murine brain death model. J Surg Res, 2013. 183(1): p. 419-26.

73. Schuurs, T.A., et al., Time-dependent changes in donor brain death related processes. Am J Transplant, 2006. 6(12): p. 2903-11.

74. Hirayama, S., et al., Prevention of neutrophil migration ameliorates rat lung allograft rejection. Mol Med, 2006. 12(9-10): p. 208-13.

75. Kunugi, S., et al., The pathological characteristics of acute antibody-mediated rejection in DA-to-Lewis rat orthotopic liver transplantation. Transplant Proc, 2011. 43(7): p. 273740.

76. Li, S., et al., Reduction of cold ischemia-reperfusion injury by graft-expressing clusterin in heart transplantation. J Heart Lung Transplant, 2011. 30(7): p. 819-26.

77. Jassem, W., et al., Leukocyte infiltration and inflammatory antigen expression in cadaveric and living-donor livers before transplant. Transplantation, 2003. 75(12): p. 2001-7.

78. Jassem, W., et al., Non-heart-beating versus cadaveric and living-donor livers: differences in inflammatory markers before transplantation. Transplantation, 2003. 75(8): p. 1386-90.

79. dos Santos, D.C., et al., Mononuclear inflammatory infiltrate and microcirculation injury in acute rejection: role in renal allograft survival. Ren Fail, 2013. 35(5): p. 601-6. 
80. Winter, J.B., et al., Distinct phenotypes of infiltrating cells during acute and chronic lung rejection in human heart-lung transplants. Transplantation, 1995. 59(1): p. 63-9.

81. Sadeghi, M., et al., Decreasing plasma soluble IL-1 receptor antagonist and increasing monocyte activation early post-transplant may be involved in pathogenesis of delayed graft function in renal transplant recipients. Clin Transplant, 2010. 24(3): p. 415-23.

82. Kanno, S., et al., Macrophage accumulation associated with rat cardiac allograft rejection detected by magnetic resonance imaging with ultrasmall superparamagnetic iron oxide particles. Circulation, 2001. 104(8): p. 934-8.

83. Zingarelli, B., et al., Lung injury after hemorrhage is age dependent: role of peroxisome proliferator-activated receptor gamma. Crit Care Med, 2009. 37(6): p. 1978-87.

84. Chima, R.S., et al., C-peptide ameliorates kidney injury following hemorrhagic shock. Shock, 2011. 35(5): p. 524-9.

85. Menezes, J., et al., A novel nitric oxide scavenger decreases liver injury and improves survival after hemorrhagic shock. Am J Physiol, 1999. 277(1 Pt 1): p. G144-51.

86. Richter, J.R., et al., Macrophage-derived chemokine (CCL22) is a novel mediator of lung inflammation following hemorrhage and resuscitation. Shock, 2014. 42(6): p. 525-31.

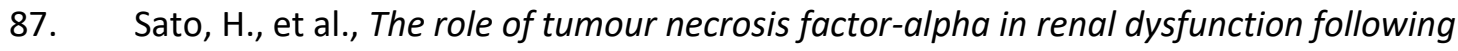
mild haemorrhage in rats. Int J Exp Pathol, 2004. 85(6): p. 345-53.

88. Jordan, J.E., Z.Q. Zhao, and J. Vinten-Johansen, The role of neutrophils in myocardial ischemia-reperfusion injury. Cardiovasc Res, 1999. 43(4): p. 860-78.

89. Formigli, L., et al., Are macrophages involved in early myocardial reperfusion injury? Ann Thorac Surg, 2001. 71(5): p. 1596-602.

90. Novitzky, D., Detrimental effects of brain death on the potential organ donor. Transplant Proc, 1997. 29(8): p. 3770-2.

91. Avlonitis, V.S., et al., Pulmonary transplantation: the role of brain death in donor lung injury. Transplantation, 2003. 75(12): p. 1928-33.

92. Breithaupt-Faloppa, A.C., et al., Sex-related differences in lung inflammation after brain death. J Surg Res, 2016. 200(2): p. 714-21.

93. Quadri, S.M., et al., Caspase inhibition improves ischemia-reperfusion injury after lung transplantation. Am J Transplant, 2005. 5(2): p. 292-9.

94. Zhou, H., et al., Hydrogen inhalation decreases lung graft injury in brain-dead donor rats. J Heart Lung Transplant, 2013. 32(2): p. 251-8.

95. Avlonitis, V.S., et al., Early hemodynamic injury during donor brain death determines the severity of primary graft dysfunction after lung transplantation. Am J Transplant, 2007. 7(1): p. 83-90.

96. Zweers, N., et al., Donor brain death aggravates chronic rejection after lung transplantation in rats. Transplantation, 2004. 78(9): p. 1251-8.

97. Muller, W.A., Getting leukocytes to the site of inflammation. Vet Pathol, 2013. 50(1): p. 7-22.

98. van Buul, J.D., et al., ICAM-1 clustering on endothelial cells recruits VCAM-1. J Biomed Biotechnol, 2010. 2010: p. 120328.

99. Cook-Mills, J.M., M.E. Marchese, and H. Abdala-Valencia, Vascular cell adhesion molecule-1 expression and signaling during disease: regulation by reactive oxygen species and antioxidants. Antioxid Redox Signal, 2011. 15(6): p. 1607-38.

100. Kamochi, M., et al., P-selectin and ICAM-1 mediate endotoxin-induced neutrophil recruitment and injury to the lung and liver. Am J Physiol, 1999. 277(2 Pt 1): p. L310-9. 
101. Kobbe, P., et al., IL-10 administration attenuates pulmonary neutrophil infiltration and alters pulmonary iNOS activation following hemorrhagic shock. Inflamm Res, 2009. 58(3): p. 170-4.

102. Matsuo, S., et al., Single-dose rosuvastatin ameliorates lung ischemia-reperfusion injury via upregulation of endothelial nitric oxide synthase and inhibition of macrophage infiltration in rats with pulmonary hypertension. J Thorac Cardiovasc Surg, 2015. 149(3): p. 902-9.

103. Okamoto, T., et al., Nebulized nitrite protects rat lung grafts from ischemia reperfusion injury. J Thorac Cardiovasc Surg, 2013. 145(4): p. 1108-16.

104. Sheridan, B.C., et al., L-arginine prevents lung neutrophil accumulation and preserves pulmonary endothelial function after endotoxin. Am J Physiol, 1998. 274(3 Pt 1): p. L33742.

105. Caty, M.G., et al., Evidence for tumor necrosis factor-induced pulmonary microvascular injury after intestinal ischemia-reperfusion injury. Ann Surg, 1990. 212(6): p. 694-700.

106. Simas, R., et al., Mesenteric hypoperfusion and inflammation induced by brain death are not affected by inhibition of the autonomic storm in rats. Clinics (Sao Paulo), 2015. 70(6): p. 446-52.

107. Blikslager, A.T., et al., Restoration of barrier function in injured intestinal mucosa. Physiol Rev, 2007. 87(2): p. 545-64.

108. Awad, A.S., et al., Compartmentalization of neutrophils in the kidney and lung following acute ischemic kidney injury. Kidney Int, 2009. 75(7): p. 689-98.

109. Auraen, H., et al., Multiorgan procurement increases systemic inflammation in brain dead donors. Clin Transplant, 2013. 27(4): p. 613-8.

110. Saat, T.C., et al., A comparison of inflammatory, cytoprotective and injury gene expression profiles in kidneys from brain death and cardiac death donors. Transplantation, 2014. 98(1): p. 15-21.

111. Vergoulas, G., P. Boura, and G. Efstathiadis, Brain dead donor kidneys are immunologically active: is intervention justified? Hippokratia, 2009. 13(4): p. 205-10.

112. Hoeger, S., et al., Dopamine treatment in brain-dead rats mediates anti-inflammatory effects: the role of hemodynamic stabilization and D-receptor stimulation. Transpl Int, 2007. 20(9): p. 790-9.

113. Davis, A.E., 3rd, P. Mejia, and F. Lu, Biological activities of C1 inhibitor. Mol Immunol, 2008. 45(16): p. 4057-63.

114. Hoeger, S., et al., Vagal stimulation in brain dead donor rats decreases chronic allograft nephropathy in recipients. Nephrol Dial Transplant, 2014. 29(3): p. 544-9.

115. Hurt, R.T., et al., Preservation of hepatic blood flow by direct peritoneal resuscitation improves survival and prevents hepatic inflammation following hemorrhagic shock. Am J Physiol Gastrointest Liver Physiol, 2012. 303(10): p. G1144-52.

116. Diseases, N.I.o.D.a.D.a.K., Kidney Disease Statistics for the United States. 2010: Bethesda, MD.

117. Gondos, A., et al., Kidney graft survival in Europe and the United States: strikingly different long-term outcomes. Transplantation, 2013. 95(2): p. 267-74.

118. Sellares, J., et al., Understanding the causes of kidney transplant failure: the dominant role of antibody-mediated rejection and nonadherence. Am J Transplant, 2012. 12(2): p. 388-99.

119. Skrabal, C.A., et al., Organ-specific regulation of pro-inflammatory molecules in heart, lung, and kidney following brain death. J Surg Res, 2005. 123(1): p. 118-25. 
120. van der Hoeven, J.A., et al., Relationship between duration of brain death and hemodynamic (in)stability on progressive dysfunction and increased immunologic activation of donor kidneys. Kidney Int, 2003. 64(5): p. 1874-82.

121. Leeuwenberg, J.F., et al., E-selectin and intercellular adhesion molecule-1 are released by activated human endothelial cells in vitro. Immunology, 1992. 77(4): p. 543-9.

122. Rothlein, R., et al., Induction of intercellular adhesion molecule 1 on primary and continuous cell lines by pro-inflammatory cytokines. Regulation by pharmacologic agents and neutralizing antibodies. J Immunol, 1988. 141(5): p. 1665-9.

123. Ysebaert, D.K., et al., Identification and kinetics of leukocytes after severe ischaemia/reperfusion renal injury. Nephrol Dial Transplant, 2000. 15(10): p. 1562-74.

124. Langenberg, C., et al., Renal histopathology during experimental septic acute kidney injury and recovery. Crit Care Med, 2014. 42(1): p. e58-67.

125. Baggiolini, M., A. Walz, and S.L. Kunkel, Neutrophil-activating peptide-1/interleukin 8, a novel cytokine that activates neutrophils. J Clin Invest, 1989. 84(4): p. 1045-9. 


\section{LIST OF ABBREVIATIONS}

${ }^{\circ} \mathrm{C}$, degrees Celsius

$\mu \mathrm{L}$, microliters

$\mu \mathrm{m}$, micrometers

APC, allophycocyanin

ATP adenosine triphosphate

$\mathrm{BMP}$, beats per minute

cc, cubic centimeter

$\mathrm{CD}$, cluster of differentiation

CMP, complete metabolic panel

$\mathrm{CR}$, conventional resuscitation

CXCR2, C-X-C motif chemokine receptor 2

$\mathrm{dL}$, deciliter

DAMPs, damage associated molecular proteins

DNA, deoxyribonucleic acid

DPBS, dulbecco's phosphate buffered saline

DPR, direct peritoneal resuscitation

ELISA, enzyme-linked immunosorbent assay

$\mathrm{F}$, french

FABP, fatty acid binding protein

FITC, fluorescein isothiocyanate 
g, grams

HA, hyaluronic acid

HMGB-1, high mobility group box 1 protein

$\mathrm{HR}$, heart rate

HSP70, heat shock protein 70

ICAM, intracellular adhesion molecule

$\mathrm{ICP}$, intracranial pressure

ICU, intensive care unit

IFN- $\gamma$, interferon $\gamma$

IHC, immunohistochemistry

IL, interleukin

IP, intraperitoneal

IV, intravenous

IVF, intravenous fluid

$\mathrm{kg}$, kilogram

$\mathrm{L}$, liters

LPS, lipopolysaccharide

MAP, mean arterial pressure

mg, milligrams

min, minutes

$\mathrm{mL}$, milliliters

$\mathrm{mmHg}$, millimeters of mercury

mmol, millimoles 
MPO, myeloperoxidase

mRNA, messenger ribonucleic acid

NBF, neutral buffered formalin

NS, normal saline

PE, phycoerythrin

PFA, paraformaldehyde

$\mathrm{pH}$, acidity

RP-1, antibody to rat neutrophils

TBI, traumatic brain injury

TGF- $\beta$, transforming growth factor $\beta$

TIVF, targeted intravenous fluid

TNF- $\alpha$, tumor necrosis factor $\alpha$

U, units

VCAM, vascular adhesion molecule

ZO-1, tight junction protein 1 


\section{CURRICULUM VITAE}

NAME: $\quad$ Jessica Lee Weaver

ADDRESS: $\quad 2727$ Riedling Drive \#8

Louisville, KY 40206

DOB: $\quad$ Charlottesville, VA - June 10, 1985

EDUCATION

\&TRAINING: $\quad$ B.S. Chemistry with specialization in Biochemistry

University of Virginia

2003-07

M.D., Doctor of Medicine

Medical College of Wisconsin

2007-11

AWARDS:

2016 ACS Committee on Trauma KY chapter Resident Paper Competition, $1^{\text {st }}$ place for trauma paper

2014 ACS Committee on Trauma KY chapter Resident Paper Competition, $2^{\text {nd }}$ place for clinical paper

2013 ACS Committee on Trauma Region IV Trauma Resident Paper Competition, $2^{\text {nd }}$ place for basic science paper

2013 Oriens Award for Careers in Trauma honorable mention, Eastern Association for the Surgery of Trauma 2013 meeting, Scottsdale, AZ

PUBLICATIONS:

Weaver JL, Kimbrough CW, Broughton-Miller K, Frisbie M, Wojcik J, Pentecost K, Bozeman MC, Nash NA, Harbrect BG. Danger on the farm: A comparison of agricultural and animal-related injuries. Accepted to Am Surg May 2016.

Weaver JL, Matheson PJ, Hurt RT, Downard CD, McClain CJ, Garrison RN, Smith JW. Direct Peritoneal Resuscitation Alters Hepatic miRNA Expression after Hemorrhagic Shock. J Am Coll Surg. 2016 Jul;223(1):68-75.

Weaver JL, Barnett RE, Patterson DE, Ramjee VG, Riedinger E, Younga J, Sepulveda EA, Keskey RC, Cheadle WG. Large bowel disease presenting as 
small bowel obstruction is associated with a poor prognosis. Am J Emerg Med. 2015.

Weaver JL, Smith JW. Direct Peritoneal Resuscitation: A Review. Int J Surg. 2015

Weaver JL, Jones W, Miller KR. Life-Threatening Splenic Rupture Following Endoscopic Retrograde Cholangiopancreatography. Am Surg. 2014 Aug;80(8):E230-1.

Weaver JL, Bradley CT, Brasel KJ. Family engagement regarding the critically ill patient. In JA Weigelt, Surgical Clinics of North America: Surgical Critical Care (p 1637-47). Philadelphia, PA.

Bradley C, Weaver J, Brasel K. Addressing access to palliative care services in the surgical intensive care unit. Surgery. 2010 Jun;147(6):871-7.

McPhie-Lalmansingh AA, Tejada LD, Weaver JL, Rissman EF. Sex chromosome complement affects social interactions in mice. Horm Behav. 2008 Sep;54(4):565-70

\section{PRESENTATIONS:}

\section{Oral Presentations}

The Effects of Direct Peritoneal Resuscitation on Leukocyte Activity in the Kidney after Brain Death. American College of Surgeons Committee on Trauma KY Chapter Resident Paper Competition, Lexington, KY, Sept 2016.

Direct Peritoneal Resuscitation Reduces Lung Injury in Brain Death through a Caspase-8-Dependent Mechanism. Central Surgical Association annual meeting, Montreal, Quebec, Canada, March 2016.

Mopeds are just as Dangerous as Motorcycles. American College of Surgeons Committee on Trauma Region IV Resident Paper Competition, Atlanta, GA, Nov 2015.

Mopeds are just as Dangerous as Motorcycles. American College of Surgeons Committee on Trauma KY Chapter Resident Paper Competition, Louisville, KY, Aug 2015.

DPR Reduces Visceral Ischemia and Inflammatory Cytokines Following Hemorrhagic Shock. Academic Surgical Congress, Las Vegas, NV, Feb 2015.

Not All Abdomens are the Same: A Comparison of Damage Control Surgery for Intra-abdominal Sepsis vs Trauma. American College of Surgeons Committee on Trauma Region IV Resident Paper Competition, Orlando, FL, Nov 2014. 
Not All Abdomens are the Same: A Comparison of Damage Control Surgery for Intra-abdominal Sepsis vs Trauma. American College of Surgeons Committee on Trauma KY Chapter Resident Paper Competition, Louisville, KY, Sep 2014.

Direct Peritoneal Resuscitation Improves Inflammation, Liver Blood Flow, and Pulmonary Edema in a Rat Model of Acute Brain Death. American College of Surgeons Committee on Trauma Region IV Trauma Paper Competition, Mobile, AL, Nov 2013.

Critically-Ill Surgical Patients Meeting Expert-Derived Consultation Guidelines Do Not Receive Palliative Care Services. Academic Surgical Congress, Fort Myers, FL, Feb 2009

\section{Poster Presentations}

Mopeds are just as Dangerous as Motorcycles. Southeastern Surgical Congress annual meeting, Atlanta, GA, Feb 2016.

Danger on the Farm: A Comparison of Animal- and Farm-related Injuries. Southeastern Surgical Congress annual meeting, Atlanta, GA, Feb 2016.

Life-Threatening Splenic Rupture Following ERCP. Southeastern Surgical Congress annual meeting, Savannah, GA, Feb 2014.

If it's not in the chart it didn't happen: Comprehensive family meeting discussions do not lead to adequate documentation. Society of Critical Care Medicine annual congress, Nashville, TN, Feb 2009.

\section{Presentation Contributing Author}

Taylor LH, Matheson PJ, Ghazi CA, Weaver JL, Downard CD, Garrison RN, Smith JW. Direct Peritoneal Resuscitation Lowers sICAM-1 and Lung IntegrinalphaL in Acute Brain Death in Rats. Academic Surgical Congress, Jacksonville, FL, Feb 2016.

Smith JW, Weaver JL, Hurt RT, Downard CD, McClain CJ, Garrison RN, Matheson PJ. Direct Peritoneal Resuscitation Reduces Inflammatory miRNAs after Hemorrhagic Shock. Western Surgical Association, Napa Valley, CA, Nov 2015. 